https://theses.gla.ac.uk/

Theses Digitisation:

https://www.gla.ac.uk/myglasgow/research/enlighten/theses/digitisation/

This is a digitised version of the original print thesis.

Copyright and moral rights for this work are retained by the author

A copy can be downloaded for personal non-commercial research or study, without prior permission or charge

This work cannot be reproduced or quoted extensively from without first obtaining permission in writing from the author

The content must not be changed in any way or sold commercially in any format or medium without the formal permission of the author

When referring to this work, full bibliographic details including the author, title, awarding institution and date of the thesis must be given

Enlighten: Theses

https://theses.gla.ac.uk/

research-enlighten@glasgow.ac.uk 


\title{
RESISTIVE EVOLUTION OF A FORCE-FREE
}

\section{PLASMA TO EQUILIBRIUM}

\author{
By \\ Amarjit Singh Gill \\ Department of Physics and Astronomy \\ University of Glasgow \\ Glasgow G12 8QQ \\ Scotland \\ United Kingdom
}

Thesis submitted for the degree of Doctor of Philosophy

December 1990

(C) A.S. Gill, 1990 
ProQuest Number: 11007608

All rights reserved

INFORMATION TO ALL USERS

The quality of this reproduction is dependent upon the quality of the copy submitted.

In the unlikely event that the author did not send a complete manuscript and there are missing pages, these will be noted. Also, if material had to be removed, a note will indicate the deletion.

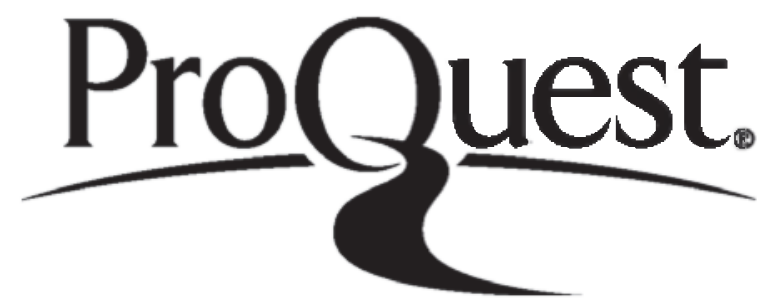

ProQuest 11007608

Published by ProQuest LLC (2018). Copyright of the Dissertation is held by the Author.

All rights reserved.

This work is protected against unauthorized copying under Title 17, United States Code Microform Edition (C) ProQuest LLC.

ProQuest LLC.

789 East Eisenhower Parkway

P.O. Box 1346

Ann Arbor, Ml $48106-1346$ 
To my family 


\section{CONTENTS}

\section{Acknowledgements}

Abstract

Chapter 1 Introduction 1

1.1 Motivation 1

1.2 MHD model 3

1.3 2D-resistive force-free evolution 5

$\begin{array}{lll}1.4 & \text { Anisotropic diffusion in a static conductor } & 10\end{array}$

$\begin{array}{lll}1.5 & \text { Taylor-Woltjer relaxation theory } & 18\end{array}$

1.6 The finite difference method 19

Figures

Chapter 2 Rectangular Boundaries 25

$2.12 \mathrm{D}$-solutions $\quad 25$

2.1.1 Stream function method 25

2.1.2 Boundary conditions and their implementation 26

2.1.3 Axial flux conservation $\quad 29$

2.1.4 Solution in terms of components of $\mathbf{B}$

2.1.5 Boundary conditions 35

2.1.6 Satisfying the divergence-free constraint 36

2.2 3D-solutions $\quad 39$

Figures 
3.1 Boundary conditions 44

3.2 Implementation of the boundary conditions 45

3.3 Axial flux conservation $\quad 48$

Figures

Chapter 4 Toroidal effects

4.1 2D-solutions $\quad 52$

4.1.1 Stream function method 52

4.1.2 Boundary conditions and their implementation 54

4.1.3 Solution in terms of components of B 55

4.1.4 Boundary conditions 56

$\begin{array}{lll}4.2 & 3 \mathrm{D} \text {-solutions } & 58\end{array}$

Figures

Chapter 5 Results and Conclusions $\quad 61$

Figures

$\begin{array}{lr}\text { Appendix A } & 83\end{array}$

$\begin{array}{lr}\text { Appendix B } & 89\end{array}$

$\begin{array}{ll}\text { Appendix C } & 94\end{array}$

$\begin{array}{ll}\text { Appendix D } & 101\end{array}$

$\begin{array}{ll}\text { References } & 110\end{array}$ 


\section{Acknowledgements}

The never-ending moral support of my mother and the rest of the family during all stages of my academic work will be forever appreciated.

I would like to thank my supervisor prof. E W Laing for setting up the project, for his help in completing my research and the effort that he put into recognising errors in the thesis. I am indebted to Dr J Cumming for the time and energy he spent in checking through every single page of this thesis. The helpful criticisms and suggestions were much appreciated. I take full responsibility for any outstanding errors. I would also like to thank Dr D A Diver for making sure that the plasma physics group computer remained faultless and for all the many useful discussions which took place between us.

Numerous useful discussions which took place with many friends in the theory groups will be well remembered.

I am grateful to the department of Physics and Astronomy of Glasgow University and the Rutherford Appleton Laboratory for providing the computing and all other necessary facilities which enabled me to carry out my research.

The research was funded by the Science and Engineering Research Council. 


\section{Abstract}

Using the magnetohydrodynamic description, the evolution of a resistive plasma can be represented as a relaxation through a sequence of force-free equilibrium states. We consider laboratory based plasmas confined in closed vessels and numerically simulate this evolution process using the finite difference method. The work is motivated by the nuclear fusion project.

We proceed by showing that the force-free problem can be reduced, in $2 D$, to solving a non-trivial $1 D$ diffusion equation subject to the forcefree constraint. Next, the diffusion of magnetic field lines is considered in a stationary 'mathematical' solid in which the magnetic field lines evolve such that the ratio of the conductivity perpendicular to the field lines to that parallel is much smaller than unity. The two processes are shown to be equivalent. Solutions to the latter problem are much easier to obtain and will be considered in chapters which follow.

We initially consider $2 D$ and $3 D$ cylindrical containment devices possessing rectangular cross-sections and develop algorithms to modet numerically the evolution of a plasma until it reaches a relaxed state. The relaxed or equilibrium profile is the most suitable state for thermonuclear fusion to proceed. 
developed. This can be used to make comparisons with experimental results for devices which have circular, D-shape and the more claborate multipinch cross-sections.

The $2 D$ and $3 D$ rectangular cross-section cases are generalised to take account of toroidal effects.

In the final chapter we present the results predicted by the suite of codes which total $\approx 20,000$ lines of source written in standard FORTRAN 77. We make comparisons with experimental data, Taylor's theory and any relevant simulations. Initially we consider a square cross-section and a variety of universal curves are found which are qualitatively similar to Taylor's theory and experiment. One of these is the well known $F \theta$ profile. The field reversal value $\theta$ is found to be a factor of two greater than that predicted by Taylor's theory but is in good agreement with a recent simulation. The universal curves predict that the final equilibrium state is completely defined once the axial flux and driving field are prescribed. These are just initial conditions and correspond to the axial flux and global helicity which are the only two quantities that need to be prescribed in Taylor's theory.

Another universal curve predicts that at a critical axial flux there are states which are inaccessible by the plasma. About this critical value there are two modes at which the plasma can be driven, one at high current and the other at low current. This corresponds to the stable high and low current mode of operation arising in Tokamaks. 
We have also found the existence of degenerate relaxed states and equilibria which carry the same current but different axial flux. Choosing a particular energy or current profile to obtain an equilibrium state may not therefore give a unique solution.

Changing the dimensions of the rectangular cross-section is found to have little effect on the universal $F \theta$ profiles.

The boundary conditions, obtained using Ohm's Law, are found to play a critical role in defining relaxed states. If we allow tangential currents to flow at a boundary, we find that the results correspond to the force-free paramagnetic model. The model where currents are removed is able to yield equilibrium states in which the field is reversed at the boundary. These are the states which arise in reversed field pinches.

Toroidal effects are found to have very little effect on $F \theta$ profiles. Other profiles do differ and from these we have found a critical aspect-ratio at which toroidal devices should be built. At this value, the maximum current can be generated for a fixed driving field.

The $3 D$ code gives rise to axisymmetric relaxed states and does not predict current limitation for any $\theta$. The curved boundary code is found to be numerically unstable for curved but non-circular cross-sections. 


\section{Chapter 1}

\section{Introduction}

\subsection{Motivation}

The realisation of producing vast amounts of energy by the phenomenon of thermonuclear fusion [1.1] in an economically viable and environmentally friendly manner is the motivation behind this project. The process involves fusing light nuclei to form heavier ones which results in large amounts of thermal energy being liberated. The reaction which occurs at the lowest ignition temperature and hence easiest to achieve is that between the two hydrogen isotopes deuterium $\mathrm{D}\left({ }^{2} \mathrm{H}\right)$ and tritium $\mathrm{T}\left({ }^{3} \mathrm{H}\right)$ :

$$
\mathrm{D}+\mathrm{T} \rightarrow\left({ }^{4} \mathrm{He}+3.5 \mathrm{MeV}\right)+(\mathrm{n}+14.1 \mathrm{MeV})
$$

Deuterium is a naturally and abundantly occurring element in water which can easily be isolated. The disadvantage of this reaction is that tritium does not occur naturally ( half life $\approx 12.5$ years ) and so has be manufactured. This can be accomplished, using lithium, through the reactions :

$$
\begin{gathered}
{ }^{6} \mathrm{Li}+\mathrm{n} \rightarrow(\mathrm{T}+2.1 \mathrm{MeV})+\left({ }^{4} \mathrm{He}+2.7 \mathrm{MeV}\right) \\
{ }^{7} \mathrm{Li}+\mathrm{n} \rightarrow \mathrm{T}+{ }^{4} \mathrm{He}+\mathrm{n}-2.5 \mathrm{MeV}
\end{gathered}
$$


Such possibilities are being investigated by experimentalists using a variety of methods and devices. One of the most promising involves the use of toroidal chambers known as TOKAMAKS [1.2]. The method essentially involves heating the hydrogen isotopes to a temperature greater than one hundred million degrees centigrade, the resulting ionised gas or plasma being contained by the use ${ }_{h}$ magnetic fields. Once the ignition temperature is reached the fusion reactions would begin. The containment vessel is to be surrounded by a blanket of lithium which would capture the neutrons breeding the required tritium and generating additional heat. The only waste product that would remain is the helium gas.

Trying to achieve the necessary conditions for thermonuclear fusion to proceed has given rise to numerous problems. The most obvious one is trying to reach the enormous temperatures that are necessary for fusion. Another problem is containing the plasma in a suitable manner. This is important because the plasma is capable of vapourising the containing vessel and thus cooling the plasma. It is therefore important to have the plasma in a dynamically stable configuration. Another problem to overcome is containing the plasma for a long enough time, the confinement time, for reactions to yield a net output of energy. This involves making up for energy losses which are lost mainly as electromagnetic radiation. The necessary condition can be expressed as a product of the plasma confinement time $\tau$ and density $\cdot$. This condition, called the Lawson criterion [1.3 ], requires $n \tau \approx 10^{22} \mathrm{~m}^{-3} \mathrm{sec}$ for a plasma at temperatures mentioned. Overcoming such problems are being investigated by theorists and experimentalists [1.2].

There are several approaches to the understanding of plasma physics. We consider here the fluid based model [1.4] which has proven to be very successful in relation to fusion studies. 


\subsection{MHD model}

The magnetohydrodynamic ( MHD) model describes the interaction of a conducting fluid with electromagnetic fields. The closed set of evolution equations consist of the energy, momentum and mass constraints of conducting-fluid dynamics including Maxwell's electromagnetic equations together with a generalised Ohm's Law. A simplified version of these evolution equations can be written [1.5] in the following form :

\section{Maxwell's equations}

$$
\begin{aligned}
& \nabla \cdot \mathbf{B}=0 \\
& \nabla \cdot \mathbf{E}=\frac{\rho}{\varepsilon_{\mathrm{o}}} \\
& \nabla \times \mathbf{E}=-\frac{\partial \mathbf{B}}{\partial t} \\
& \nabla \times \mathbf{B}=\mu_{\mathrm{o}} \mathbf{j}
\end{aligned}
$$

\section{Continuity equation}

$$
\frac{D \rho}{D t}+\rho \nabla \cdot \mathbf{V}=0
$$




\section{Momentum equation}

$$
\rho \frac{D \mathbf{V}}{D t}+\nabla P=\mathbf{j} \times \mathbf{B}
$$

\section{Energy equation}

$$
\frac{D}{D t}\left(P \rho^{-5 / 3}\right)=\frac{2}{3} \eta \rho^{-5 / 3} j^{2}
$$

\section{Ohm's Law}

$$
\mathbf{E}+\mathbf{V} \times \mathbf{B}=\eta \mathbf{j}
$$

where $\mathbf{B}, \mathbf{E}, \mathbf{V}, \mathbf{j}, \rho, P$, and $\eta$ are the magnetic field, electric field, fluid velocity, current density, plasma density, plasma pressure and plasma resistivity respectively.

In the derivation of most of these equations a large number of approximations are made [1.5]. A lot of these approximations are difficult to justify but nevertheless MHD theory has yielded an enormous amount of useful information consistent with experiment [1.6]. 


\subsection{D-resistive force-free evolution}

The non-linear nature of the MHD equations inevitably involves the use of numerical methods when seeking realistic solutions especially in two and three dimensional problems. Unfortunately, finding numerical solutions even to the simplified evolution equations is immensely difficult due mainly to the two disparate time scales, the Alfvén time ( $\approx 10^{-7}$ secs. $)$ and the resistive diffusion time ( $\approx 10$ secs. $)$. These are typical JET ( Joint European Torus ) parameters.

It was therefore proposed by Taylor et al [1.7] that the momentum equation should be replaced by the force-free equilibrium constraint $\mathbf{j} \times \mathbf{B}=0$. This corresponds to neglecting high velocity Alfvén phenomena. If $\mathbf{B}$ is written as $\left(\psi_{y},-\psi_{x}, B_{z}\right)$, where the subscript implies a partial derivative, then $B_{z}$ is necessarily a functional of $\psi$, the $z$-component of the vector potential $\mathbf{A}$, where $\mathbf{B}=\nabla \times \mathbf{A}$.

The introduction of the vector potential allows (1.3) to be integrated to give the evolution equation for $A_{z}$ :

$$
\frac{\partial A_{z}}{\partial t}=\frac{\partial \psi}{\partial t}=-E_{z}+C(t)
$$

$C(t)$ is a time dependent function arising from the integration and will be interpreted later.

From (1.4) and (1.8)

$$
E_{z}=(\mathbf{V} \cdot \nabla) \psi-\eta \nabla^{2} \psi
$$


Substitution then gives

$$
\frac{\partial \psi}{\partial t}+(\mathbf{V} \cdot \nabla) \psi=\eta \nabla^{2} \psi+C(t)
$$

Also from (1.3), (1.4) and (1.8)

$$
\frac{\partial B_{z}}{\partial t}=(\mathbf{B} \cdot \nabla) V_{z}-\nabla \cdot\left(B_{z} \mathbf{V}\right)+\eta \nabla^{2} B_{z}
$$

This evolution equation gives the time development of $B_{z}$ at fixed point in space. It is convenient to know the evolution of $B_{z}$ on a particular $\psi$ contour. This can be obtained using :

$$
\frac{\partial B_{z}}{\partial t}=\frac{\partial B_{z}}{\partial \psi} \frac{\partial \psi}{\partial t}+\left.\frac{\partial B_{z}}{\partial t}\right|_{\psi}
$$

But

$$
\frac{\partial B_{z}}{\partial x}=\frac{\partial B_{z}}{\partial \psi} \frac{\partial \psi}{\partial x} \quad \Rightarrow \quad \nabla B_{z}=B_{z}^{\prime} \nabla \psi
$$

where the prime represents $\frac{\partial}{\partial \psi}$.

Taking the divergence of $\nabla B_{z}$ results in 


$$
\nabla^{2} B_{z}=B_{z}^{\prime \prime}(\nabla \psi)^{2}+B_{z}^{\prime} \nabla^{2} \psi
$$

Using these two results and substituting from (1.10) and (1.11) gives the following evolution equation at fixed $\psi$ contour :

$$
\frac{\partial B_{z}}{\partial t}+B_{z} \nabla \cdot \mathbf{V}=\eta B_{z}{ }^{\prime \prime}(\nabla \psi)^{2}+(\mathbf{B} \cdot \nabla) V_{z}-B_{z}^{\prime} C
$$

It should be noted that :

1) only the component of $\mathbf{V}$ perpendicular to $\mathbf{B}$ is relevant.

2) $\mathbf{V}$ is a single valued function of position.

3) since $(\mathbf{B} \cdot \nabla) \psi=0$, the projection in the $x, y$ plane of the $\mathbf{B}$-lines coincide with closed $\psi$-contours.

Equations (1.10) and (1.13) can now be used to to eliminate $\mathrm{V}$ to give an evolution equation involving terms in $A$ and $I$, where :

$$
\begin{aligned}
& A=\oint_{\psi} \frac{d l}{|\nabla \psi|} \\
& I=\oint_{\psi}|\nabla \psi| d l
\end{aligned}
$$


From (1.10)

$$
\begin{aligned}
\oint_{\psi} \frac{\partial \psi}{\partial t} \frac{d l}{|\nabla \psi|} & =\left\langle\frac{\partial \psi}{\partial t}\right\rangle \\
& =\eta I^{\prime}+C A-\oint_{\psi}(\mathbf{V} \cdot \nabla) \psi \frac{d l}{|\nabla \psi|}
\end{aligned}
$$

and from (1.13)

$$
\oint_{\psi} \frac{\partial B_{z}}{\partial t} \frac{d l}{|\nabla \psi|}=A \frac{\partial B_{z}}{\partial t}=\eta B_{z}^{\prime \prime} I-C A B_{z}^{\prime}-B_{z} \oint_{\psi} \nabla \cdot \mathrm{V} \frac{d l}{|\nabla \psi|}
$$

since

$$
\oint_{\psi}(\mathbf{B} \cdot \nabla) V_{z} \frac{d l}{|\nabla \psi|}=\oint_{\psi}\left(\nabla V_{z}\right) \times(\nabla \psi) \cdot \mathbf{k} \frac{d l}{|\nabla \psi|}=\oint_{\psi} \nabla V_{z} \cdot \mathbf{d l}=0
$$

Using these two results :

$$
\begin{aligned}
\left.A \frac{\partial B_{z}}{\partial t}-B_{z}<\frac{\partial \psi}{\partial t}\right\rangle^{\prime} & =\left(\eta B_{z}^{\prime \prime} I-B_{z}^{\prime} C A\right)-B_{z}\left(C A+\eta I^{\prime}\right)^{\prime} \\
& +B_{z} \oint_{\psi}[((\mathbf{V} \cdot \nabla) \psi)-\nabla \cdot \mathbf{V}]^{\prime} \frac{d l}{|\nabla \psi|}
\end{aligned}
$$


But :

$$
\frac{(\mathbf{V} \cdot \nabla) \psi}{|\nabla \psi|}=\frac{\partial V_{\psi}}{\partial \psi}
$$

and

$$
\frac{\nabla \cdot \mathbf{V}}{|\nabla \psi|}=\frac{\partial V_{\psi}}{\partial \psi}+\frac{\partial}{\partial l}\left(\frac{V_{l}}{|\nabla \psi|}\right)
$$

where the subscripts $l$ and $\psi$ denote values along and perpendicular to $\psi$ respectively.

Since $\mathbf{V}$ is single valued it follows that :

$$
A \frac{\partial B_{z}}{\partial t}=\left[\eta\left(I B_{z}^{\prime}-I^{\prime} B_{z}\right)-C A B_{z}\right]^{\prime}+B_{z}<\frac{\partial \psi}{\partial t}>
$$

This is effectively a one dimensional diffusion equation involving averages on $\psi$ contours. It is subject to the force-free constraint $\nabla^{2} \psi+B_{z} B_{z}^{\prime}=0$.

Although this has eliminated the presence of the two disparate time scales we still have a formidable non-standard problem, involving coefficients in the diffusion equation defined by contour integrals defined by a function $\psi(x, y ; t)$ which is itself a solution ( with suitable boundary conditions ) of the non-linear force-free constraint. In this constraint $B_{z}(\psi)$ is an unknown function obtained by solving the diffusion equation. This represents a self-consistent problem of considerable 
difficulty. We next present an alternative representation of the same evolution problem.

\subsection{Anisotropic diffusion in a static rigid conductor}

We extend the work of Hobbs [1.8] on anisotropic diffusion and consider the diffusion of magnetic field lines in a stationary rigid conductor in which the electrical conductivity $\sigma_{1 \mid}$ along field lines is much greater than the cross-field conductivity $\sigma_{\perp}$. In the limit $\varepsilon=\sigma_{\perp} / \sigma_{11} \rightarrow 0$, the magnetic field diffuses through a sequence of force-free equilibrium states. We show that in this limit the equations which determine magnetic field diffusion in the stationary conductor are identical to the equations which describe the evolution of force-free magnetic fields in a resistive plasma.

The magnetic field evolves according to

$$
\frac{\partial \mathbf{B}}{\partial t}=-\nabla \times \mathbf{E}
$$

Ohm's Law can be written as

$$
\mathbf{E}=\frac{\mathbf{j}_{1}}{\sigma_{\perp}}+\frac{\mathbf{j}_{11}}{\sigma_{11}}
$$

where $\mathbf{j}=\mathbf{j}_{1}+\mathbf{j}_{11}$ and $j_{11}=\frac{(\mathbf{j} \cdot \mathbf{B}) \mathbf{B}}{B^{2}}$ 
(1.16) can then be expressed as $\mathbf{E}=a F \mathbf{B}+b \mathbf{j}$

where :

$$
\begin{aligned}
& F=-\frac{\mathbf{j} \cdot \mathbf{B}}{B^{2}}=\frac{B_{z} \nabla^{2} \psi-\nabla \psi \cdot \nabla B_{z}}{(\nabla \psi)^{2}+B_{z}{ }^{2}} \\
& a=\left[\frac{1}{\sigma_{\perp}}-\frac{1}{\sigma_{11}}\right] \text { and } b=\frac{1}{\sigma_{\perp}}
\end{aligned}
$$

Now introduce a stream function $\psi=\psi(x, y ; t)$ defined by

$$
\mathbf{B}=\left[\frac{\partial \psi}{\partial y},-\frac{\partial \psi}{\partial x}, B_{z}\right)
$$

$\psi$ is again the $z$-component of the vector potential.

Substituting into (1.15) and integrating the $\psi$ equation leads to the following pair of coupled diffusion equations :

$$
\begin{aligned}
& \frac{\partial B_{z}}{\partial t}=b \nabla^{2} B_{z}+a \nabla \cdot(F \nabla \psi) \\
& \frac{\partial \psi}{\partial t}=b \nabla^{2} \psi-a F B_{z}+C(t)
\end{aligned}
$$


where $C(t)$ can be interpreted as a driving externally applied axial electric field. which may be time-dependent.

Due to the two different values of the conductivities there exist two time scales $\tau_{!}$, and $\tau_{1}$, the first characterised by $\sigma_{11}$ and the second by $\sigma_{1}$. If $\varepsilon=\sigma_{1} / \sigma_{1} \ll 1$ then $\tau_{\perp} \ll \tau_{11}$. In such a case a rapid diffusion and equilibration takes place on the $\tau_{1}$ time scale accompanied by a much slower diffusion on the $\tau_{1}$, time scale.

The diffusion equations describing evolution on the short time scale can be written as :

$$
\begin{aligned}
& \frac{\partial B_{z}}{\partial t}=\nabla^{2} B_{z}+(1-\varepsilon) \nabla \cdot(F \nabla \psi) \\
& \frac{\partial \psi}{\partial t}=\nabla^{2} \psi-(1-\varepsilon) F B_{z}+\varepsilon \alpha
\end{aligned}
$$

where $t \rightarrow t / \sigma_{1}$ and $\alpha=\sigma_{11} C$.

We next allow the system to be in an arbitrary state and show that it must relax to some force-free stationary state in the limit $\varepsilon \rightarrow 0$. Consider the stationary states of (1.20) and (1.21):

$$
\nabla \cdot\left(F \nabla \psi+\nabla B_{z}\right)=0
$$

$$
\nabla^{2} \psi-F B_{z}=0
$$


From (1.22)

$$
F \nabla \psi+\nabla B_{z}=\nabla \times \mathbf{G}
$$

where $\mathbf{G}$ is an arbitrary vector.

The LHS of (1.24) has no z-component and so $\mathbf{G}=G \mathbf{z}$.

From (1.23) and (1.24) :

$$
\begin{aligned}
& F(\nabla \psi)^{2}=\nabla \times \mathbf{G} \cdot \nabla \psi-\nabla B_{z} \cdot \nabla \psi \\
& F\left(B_{z}\right)^{2}=B_{z} \nabla^{2} \psi
\end{aligned}
$$

Adding the above two and comparing with (1.17) gives:

$$
\nabla \times \mathbf{G} \cdot \nabla \psi=0 \Rightarrow G=G(\psi)
$$

But

$$
\nabla \times \mathbf{G}=\frac{d G}{d \psi} \nabla \psi \times \mathbf{z}
$$




$$
\nabla B_{z} \times \nabla \psi=\frac{d G}{d \psi}(\nabla \psi)^{2} \mathbf{z}
$$

Dividing by $|\nabla \psi|$, integrating around a $\psi$ contour and noting that $B_{z}$ must be single-valued, gives :

$$
\oint_{\psi} B_{z} d l=0=-\frac{d G}{d \psi} \oint_{\psi}|\nabla \psi| d l=-I \frac{d G}{d \psi}
$$

If $I(\psi) \neq 0$, then we must have $\frac{d G}{d \psi}=0$. That is $G$ must be a constant.

It then follows that $\nabla B_{z}$ is parallel to $\nabla \psi$ which means that $B_{z}$ is necessarily a functional of $\psi$.

Now the force-free constraint can be written as

$$
\mathbf{j} \times \mathbf{B}=B_{z} \nabla B_{z}+\nabla^{2} \psi \nabla \psi+\nabla B_{z} \times \nabla \psi
$$

After substituting from the stationary states and using the above result, this is shown to be zero.

This does not tell us which force-free state the system relaxes to. To consider this question we look at the evolution of the axial flux $\Phi$ on the long time scale for finite $\varepsilon$.

By definition $\Phi=\int_{S} B_{z} d S$ is the axial flux enclosed by a $\psi$ contour, where $S$ is the area bounded by the contour. 
The long time scale evolution equation can be written as

$$
\begin{aligned}
& \varepsilon \frac{\partial \psi}{\partial t}=\nabla^{2} \psi-(1-\varepsilon) F B_{z}+\varepsilon \alpha \\
& \varepsilon \frac{\partial B_{z}}{\partial t}=\nabla \cdot\left((1-\varepsilon) F \nabla \psi+\nabla B_{z}\right)
\end{aligned}
$$

Now $\left.\frac{\partial \Phi}{\partial t}\right|_{\psi}$ is made up of two contributions :

a) for a fixed $\psi$ contour ;

$$
\begin{aligned}
\varepsilon\left[\frac{\partial \Phi}{\partial t}\right]_{a} & =\int_{S} B_{z} d s \\
& =\int_{S} \nabla \cdot\left((1-\varepsilon) F \nabla \psi+\nabla B_{z}\right) d s \\
& =\oint_{\psi}\left((1-\varepsilon) F|\nabla \psi|^{2}+\nabla B_{z} \cdot \nabla \psi\right) \frac{d l}{|\nabla \psi|}
\end{aligned}
$$

b) for $\psi$ sweeping out axial flux, with velocity $-\frac{\partial \psi}{\partial t} \frac{1}{|\nabla \psi|}$;

$$
\varepsilon\left[\frac{\partial \Phi}{\partial t}\right]_{b}=-\oint_{\psi} B_{z} \frac{\partial \psi}{\partial t} \frac{d l}{|\nabla \psi|}
$$




$$
=-\oint_{\psi}\left(B_{z} \nabla^{2} \psi-(1-\varepsilon) F B_{z}^{2}+\varepsilon \alpha B_{z}\right) \frac{d l}{|\nabla \psi|}
$$

Adding both contributions and substituting for $F$ results in

$$
\frac{\partial \Phi}{\partial t}=-\oint_{\psi}\left(B_{z} \nabla^{2} \psi-\nabla \psi \cdot \nabla B_{z}+\alpha B_{z}\right) \frac{d l}{|\nabla \psi|}
$$

Thus, the system ultimately relaxes to a force-free field satisfying the constraint

$$
\oint_{\psi}\left(B_{z} \nabla^{2} \psi-\nabla \psi \cdot \nabla B_{z}+\alpha B_{z}\right) \frac{d l}{|\nabla \psi|}=0
$$

For force-free fields this reduces to

$$
I \frac{d B_{z}}{d \psi}-B_{z} \frac{d I}{d \psi}-\alpha B_{z} A=0
$$

where :

$$
\begin{aligned}
& I(\psi ; t)=\oint_{\psi}|\nabla \psi| d l \\
& A(\psi ; t)=\oint \frac{d l}{|\nabla \psi|}
\end{aligned}
$$


enclosed by neighbouring $\psi$ contours respectively.

Since we have shown that in the limit $\varepsilon \rightarrow 0$ there is rapid equilibration to forcefree fields the equation of motion for the axial flux can then be written as

$$
\frac{\partial \Phi}{\partial t}=-\left(I \frac{d B_{z}}{d \psi}-B_{z} \frac{d I}{d \psi}-\alpha B_{z} A\right)
$$

and it can be shown that :

$$
\begin{aligned}
& \left.\frac{\partial \Phi}{\partial \psi}\right|_{t}=-B_{z} \psi \\
& \left.\frac{\partial V}{\partial t}\right|_{\psi}=-\frac{\partial}{\partial \psi}\left(\oint_{\psi} \frac{\partial \psi}{\partial t} \frac{d l}{|\nabla \psi|}\right)
\end{aligned}
$$

It then follows that

$$
\left.\frac{\partial B_{z}}{\partial t}\right|_{\psi} A-B_{z} \frac{\partial}{\partial \psi}\left(\oint_{\psi} \frac{\partial \psi}{\partial t} \frac{d l}{|\nabla \psi|}\right)=-\left(I \frac{d B_{z}}{d \psi}-B_{z} \frac{d I}{d \psi}-\alpha B_{z} A\right)^{\prime}
$$

This is identical to (1.14) the equation of force-free evolution in a resistive pressureless plasma.

When obtaining solutions to the force-free evolution problem we do not of course solve the evolution equation (1.25) but the two much simpler diffusion equations (1.20) and (1.21) with a suitable choice for $\varepsilon$ and $\alpha$. This will be considered in the 
next three chapters.

\subsection{Taylor-Woltjer theory of relaxed states}

The evolution of an ideal plasma bounded by a perfectly conducting vessel has been considered by Woltjer [1.9]. He was able to show that the helicity $K$ defined by

$$
K=\int_{\tau} \mathbf{A} \cdot \mathbf{B} d \tau \quad \tau \equiv \text { volume of vessel }
$$

was an invariant of motion of the system. The perfectly conducting boundary introduces an additional constraint namely that the axial flux also remains an invariant of motion. Then by minimising the magnetic energy, subject to the helicity and axial flux constraints, he was able to show that the stationary or equilibrium profile reached by the plasma would be the force-free state $\nabla \times \mathbf{B}=\mu \mathbf{B}, \mu$ being a constant. The helicity invariant is in fact an infinity of invariants, one for each field line.

The same problem was considered by Taylor [1.10] who proposed that for a plasma with infinitesimal but finite resistivity, the infinity of invariants introduced by Woltjer's theory could be approximated by a single global invariant, the global helicity. Taylor was then able to find a minimum magnetic energy state subject to the global helicity and axial flux constraints. He also found the relaxed state to be force-free.

For a circular cross-section toroidal device of large aspect ratio the cylindrically symmetric solutions to the force-free constraint can be written as 


$$
B_{r}=0, B_{\theta}=B_{0} J_{0}(\mu r) \text { and } B_{z}=B_{0} J_{1}(\mu r)
$$

which for obvious reasons is referred to as the Bessel Function Model ( BFM ).

Experimental data is sometimes represented by $F-\theta$ diagrams, where $F$ and $\theta$ are the ratios of the toroidal and poloidal fields at the boundary to the mean toroidal field across the cross-section, they are usually referred to as the field reversal parameter and the pinch ratio respectively.

Figure 1.1 ( end of first Chapter) shows several points on the $F-\theta$ diagram obtained by experiment and the corresponding results predicted by the BFM $[1.11$, 1.12]. As can be seen, there is remarkable agreement between theory and experiment. The predicted field profiles are also shown, again there is very good agreement. Taylor's theory will then be very useful for comparison purposes especially when experimental data is unavailable.

It should be pointed out that Taylor's theory can only be used to determine equilibrium profiles and in no way describes the mechanism of relaxation.

It should also be mentioned that there are several other remarkable features of toroidal discharges predicted by Taylor's theory; and will be referred to in later chapters.

\subsection{The finite difference method}

This method represents a very powerful approach for obtaining numerical solutions to differential equations which may be simple or very difficult to solve $|1.13|$. It involves replacing continua and derivatives by discrete approximations. For 
example, consider obtaining the solution of the following very simple diffusion equation :

$$
\frac{\partial U}{\partial t}=\frac{\partial^{2} U}{\partial x^{2}}
$$

along the region $a b$ ( figure 1.2) and subject to the boundary conditions $U(x=a ; t)=U_{a}$ and $U(x=b ; t)=U_{b}$ with initial condition $U(x ; t=0)=U_{0}$. The region $a b$ can then be discretised by assuming that information $U_{i}$ only along the mesh points $i$ is available. Approximations to derivatives can then be obtained using Taylor expansions. Replacing the spatial derivative by a second order and the temporal derivative by a first order approximation gives the difference equation

$$
\frac{U_{i}^{n+1}-U_{i}^{n}}{\Delta t}+\mathrm{O}(\Delta t)=\frac{U_{i+1}^{n}-2 U_{i}^{n}+U_{i-1}^{n}}{2 \Delta x}+\mathrm{O}\left(\Delta x^{2}\right)
$$

where the superscript $n$ refers to temporal discretisation.

Now, $U$ is known initially $(n=0)$ so the updated value, at $n=1$, can be obtained at all mesh points from $i=2$ to $i=m-1$. The value of $U$ on the boundary is fixed by the boundary conditions. The solution at $n=1$ can then be used to obtain values at $n=2$. The procedure is repeated as long as is necessary.

The PDE could also be written as

$$
\frac{\partial U}{\partial t}=\frac{\partial}{\partial x}\left(\frac{\partial U}{\partial x}\right)
$$


and could then be replaced by the difference equation

$$
\frac{U_{i}^{n+1}-U_{i}^{n}}{\Delta t}+O(\Delta t)=\frac{U_{i+2}^{n}-2 U_{i}^{n}+U_{i-2}^{n}}{4 \Delta x^{2}}+O\left(\Delta x^{2}\right)
$$

which can also be used to update $U$ but would involve using information at two neighbouring mesh points when updating a point. It also requires extrapolations across the boundary in order to update interior boundary neighbours ( points adjacent to boundary where a solution is required). The choice of difference equation will usually depend on the problem at hand.

The above methods work for the Dirichlet boundary conditions being considered. For Neumann boundary conditions fictitious exterior boundary points can be introduced. Consider the above problem but with the boundary conditions

$$
\frac{\partial}{\partial x} U(x=a ; t)=U_{\mathrm{o}} \text { and } \frac{\partial}{\partial x} U(x=b ; t)=-U_{\mathrm{o}}
$$

The condition at $a$ can be replaced by

$$
U_{1}-U_{-1}=2 \Delta x U_{0}
$$

This difference equation then defines the unknown exterior boundary neighbour $U_{-1}$ which can be used for updating the boundary value.

A simpler but less accurate method, first order, would be to use the forward difference formula 


$$
U_{1}-U_{0}=\Delta x U_{0}
$$

then instead of using the evolution equation the boundary condition would be used to update the boundary value. Once again the method used usually depends on the nature of the problem.

Updated values obtained using the above difference equations depend only on information at the preceding time. Such methods are referred to as explicit schemes. Other schemes requiring information at the advanced time are called implicit schemes. The unfortunate problem with explicit schemes is that they place a restriction on the time step. If the time step is too large the solution becomes numerically unstable. The restriction known as the CFL ( Courant-Friedrichs-Levy ) condition can be written, for the above equivalent of a $3 \mathrm{D}$ problem, as

$$
\Delta t \leq \frac{1}{2\left(\frac{1}{\Delta x^{2}}+\frac{1}{\Delta y^{2}}+\frac{1}{\Delta z^{2}}\right)}
$$

This condition will obviously lead to computational problems if solutions over long times are required. The above stability condition arises when considering the simple diffusion equation. For non-linear problems it is usually too difficult to find the stability criterion and the only way to see if the numerical algorithm works is to try it out. When deriving the CFL condition no account is taken of the nature of the boundary condition. In fact the stability of a numerical scheme has a strong dependence on the form of the boundary condition. Consider obtaining a solution of the simple diffusion equation

$$
\frac{\partial U}{\partial t}=\frac{\partial^{2} U}{\partial x^{2}}
$$


with the time dependent boundary conditions

$$
\frac{\partial}{\partial x} U(x=a ; t)=U(x=a ; t) \text { and } \frac{\partial}{\partial x} U(x=b ; t)=-U(x=b ; t)
$$

Replacing by second order difference formulae gives

$$
\begin{aligned}
& U_{i}^{n+1}=U_{i}^{n}+\frac{\Delta t}{\Delta x^{2}}\left(U_{i+1}^{n}-2 U_{i}^{n}+U_{i-1}^{n}\right) \\
& U_{1}-U_{-1}=2 \Delta x U_{0}
\end{aligned}
$$

For this problem the stability condition would not be the CFL condition but $\mid 1.14]$

$$
\Delta t \leq \frac{1}{2\left(\frac{1}{\Delta x^{2}}+\frac{1}{2 \Delta x}\right]}
$$

so the time step limit is further reduced.

When considering long time simulations it is essential to ensure that the solution remains accurate in time. It is therefore necessary to use algorithms which are second order accurate in time rather than first order as above. To see how second order accuracy can be obtained [1.15] consider the solution to

$$
\frac{\partial}{\partial t} U(x ; t)=f(x ; t)
$$


Taking forward differences and advancing by half a time step results in

$$
U^{n+\frac{1}{2}}=U^{n}+\frac{1}{2} \Delta t f^{n}+\mathrm{O}\left(\Delta t^{2}\right)
$$

With central differences, centering mid-way between a time step gives

$$
U^{n+1}=U^{n}+\Delta t f^{n+\frac{1}{2}}+O\left(\Delta t^{3}\right)
$$

Once $U^{n+\frac{1}{2}}$ has been calculated it can be used to obtain $f^{n+\frac{1}{2}}$. The data $U^{n}$ and $f^{n+\frac{1}{2}}$ is then used to obtain the required $U^{n+1}$ values. This method is usually referred to as a predictor-corrector scheme.

Implicit schemes do not have the CFL time step restriction but these schemes are usually very difficult to implement and involve time consuming iterations in obtaining a solution. Such schemes will not be considered.

The finite difference method has been used extensively in the study of the MHD equations and has been successful in numerous simulations [1.16]. 

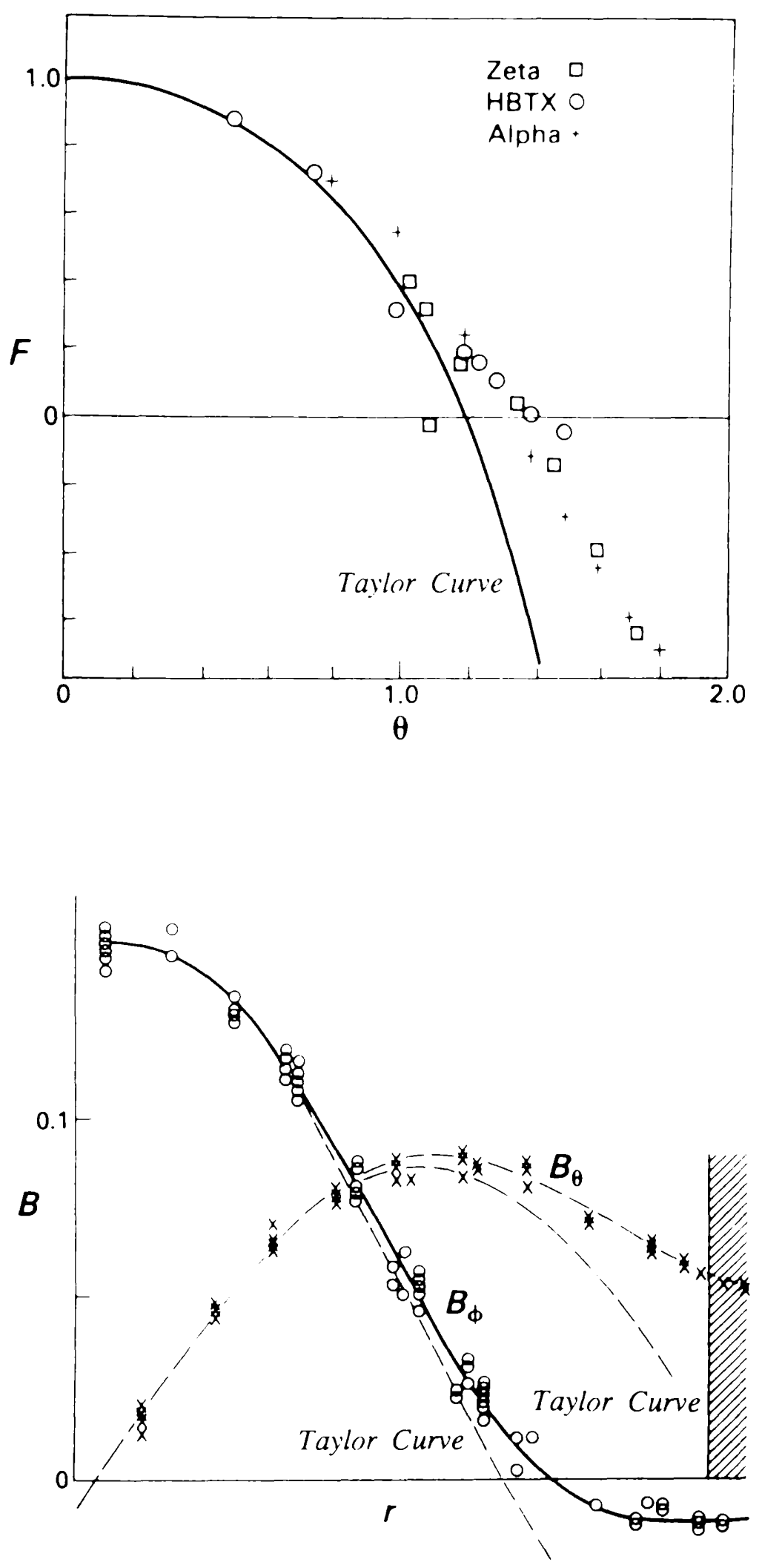

Figure 1.1

Universal $F$ versus $\theta$ profile predicted by Taylor's theory ( top ) together with experimental results ( from Bodin and Newton, 1980). Also shown (bottom) are the toroidal and poloidal field profiles ( from Bodin, 1984 ). 

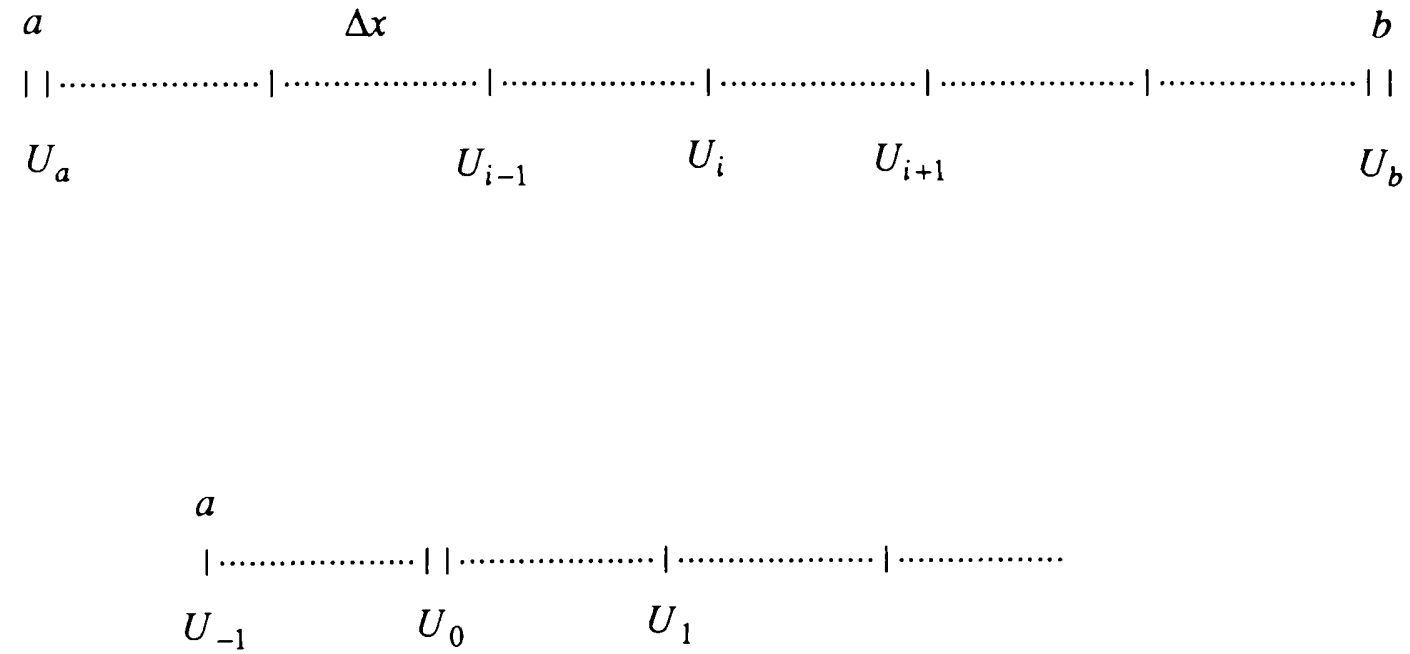

Figure 1.2

Continuum $a b$ ( top ) over which solution is to be obtained is replaced by the discrete mesh of points $i$ separated by a mesh spacing of $\Delta x$. When updating a boundary value $U_{0}$ ( bottom ), the exterior boundary neighbour $U_{-1}$ is used. 


\section{Chapter 2}

\section{Rectangular Boundaries}

The finite difference method as described in section 1.6 will be used to obtain numerical solutions to the short time scale evolution equations (1.20) and (1.21). A variety of methods solving the same problem will be presented. This is essential for cross-referencing purposes since numerically there is usually more than one approach in obtaining a solution. As a first step rectangular boundaries will be considered as this ensures that the finite difference representations remain relatively simple at an interface. The task of implementing Neumann type boundary conditions then becomes straight forward. To simplify matters further it is assumed that the solutions are independent of the $z$ co-ordinate.

\section{$2.1 \quad$ 2D-Solutions}

\subsubsection{Stream function method}

The use of the stream function $\psi$ simplifies matters in two ways. Firstly, it reduces the number of evolution equations by one and so less effort is needed in coding and numerical solutions are obtained more rapidly. Secondly, the stream function is defined such that the divergence-free constraint is satisfied automatically.

Consider the solid to be in the form of an infinitely long rectangular cylinder and surrounded by a perfectly conducting shell. The evolution equations (1.20) and (1.21) are then to be solved subject to the boundary conditions imposed by the 
perfectly conducting shell.

\subsubsection{Boundary conditions and their implementation}

Before seeking solutions, the boundary conditions must be obtained for $B_{z}$ and $\psi$. Since the solid is assumed to be surrounded by a perfectly conducting shell the normal component of the magnetic field and the tangential component of the electric field must vanish at the interface. It also introduces an additional global constraint namely that the axial flux be an invariant of motion.

At an interface $\mathbf{n} \cdot \mathbf{B}=0 \Rightarrow \psi=\psi(t)$ only.

Equation (1.19) can be written as :

$$
\frac{\partial \psi}{\partial t}=-E_{z}+C(t)
$$

In general, the term $C$ is a function of time and corresponds to an electric field. If $\psi$ on the boundary is then kept constant throughout the evolution it can be interpreted as a time and space independent axial electric driving field applied at the rim. We take this to be constant. This means that $\alpha$ in (1.21) is then just a driving term.

The axial driving field is tangential to the surface of the solid which is of course inconsistent with the boundary conditions. In practice the driving field would be applied across a poloidal gap in the perfectly conducting shell. But this allows axial flux to leave through the gap and hence violate the flux constraint. The inconsistency is to be considered as an approximation as we are considering only a 
model. How realistic the model is to an experimental device can only be determined by the results it produces. This model has also been considered, looking at similar evolutions, by others $[2.1,2.2]$ and has given realistic results and so will be used here.

Consider figure 2.1. Using Ohm's Law, it can be shown that $\{$ Appendix C \}, $\mathbf{n} \times \mathbf{E}=0 \Rightarrow$

$$
\begin{aligned}
& \frac{\partial B_{z}}{\partial x}=\frac{\alpha(1-\varepsilon) B_{z} \psi_{\cdot x}}{\psi_{\cdot x}^{2}+B_{z}^{2}} \quad \text { where } \quad \psi_{\cdot x} \equiv \frac{\partial \psi}{\partial x} \\
& \frac{\partial^{2} \psi}{\partial x^{2}}=-\alpha \frac{\left(\varepsilon \psi_{\cdot x}^{2}+B_{z}^{2}\right)}{\psi_{\cdot x}^{2}+B_{z}^{2}}
\end{aligned}
$$

on ad, bc and :

$$
\begin{aligned}
& \frac{\partial B_{z}}{\partial y}=\frac{\alpha(1-\varepsilon) B_{z} \psi_{: y}}{\psi_{; y}^{2}+B_{z}^{2}} \\
& \frac{\partial^{2} \psi}{\partial y^{2}}=-\alpha \frac{\left(\varepsilon \psi_{; x}^{2}+B_{z}^{2}\right)}{\psi_{; y}^{2}+B_{z}^{2}}
\end{aligned}
$$

on $a b$ and $c d$.

There are then three boundary conditions to be satisfied at an interface. This was to be expected as originally there were three evolution equations, one for each 
component of $\mathbf{B}$. These required a boundary condition to fix each component at an interface.

The above conditions define the normal derivatives of the components of $\mathbf{B}$ at the interface. They can be implemented by introducing fictitious exterior boundary neighbours. It should be noted that these boundary conditions allow for currents to flow at an interface, ie $\mathbf{n} \times \mathbf{j} \neq 0$ on the boundary. Setting $\varepsilon$ equal to unity in these conditions is equivalent to having a current-free interface.

Consider the replacement of the derivatives by central differences. Then at the interface $a b$ the difference equations become:

$$
\begin{aligned}
& \left(B_{z}\right)_{i+1}-\left(B_{z}\right)_{i-1}=\frac{4 \alpha \Delta x^{2}(1-\varepsilon) B_{z}\left(\psi_{i+1}-\psi_{i-1}\right)}{\left(\psi_{i+1}-\psi_{i-1}\right)^{2}+B_{z}{ }^{2}} \\
& \psi_{i+1}-2 \psi+\psi_{i-1}=\frac{-\alpha \Delta x^{2}\left(\varepsilon\left(\psi_{i+1}-\psi_{i-1}\right)^{2}+\left(2 \Delta x B_{z}\right)^{2}\right)}{\left(\left(\psi_{i+1}-\psi_{i-1}\right)^{2}+\left(2 \Delta x B_{z}\right)^{2}\right)}
\end{aligned}
$$

from which follows the cubic $\psi_{i-1}^{3}+P \psi_{i-1}^{2}+Q \psi_{i-1}+R=0$

where :

$$
\begin{aligned}
& P=\varepsilon \alpha \Delta x^{2}-2 \psi-\psi_{i+1} \\
& Q=2 \Delta x^{2}\left(2 B_{z}{ }^{2}-\varepsilon \alpha \psi_{i+1}\right)+\psi_{i+1}\left(4 \psi-\psi_{i+1}\right)
\end{aligned}
$$




$$
R=\Delta x^{2}\left(4 B_{z}^{2}\left(\alpha \Delta x^{2}-\psi+\psi_{i+1}\right)+\alpha \varepsilon \psi_{i+1}^{2}\right)+\psi_{i+1}^{2}\left(\psi_{i+1}-2 \psi\right)
$$

The subscript $i, j$ has been dropped for convenience and will be done so where necessary.

A real solution of this cubic then gives the unknown exterior boundary neighbour.

A second approach is to replace the second order derivatives by central differences and first order ones by forward differences which then gives a much simpler expression for the unknown :

$$
\psi_{i-1}=2 \psi-\psi_{i+1}-\frac{\alpha \Delta x^{2}\left(\varepsilon P+B_{z}^{2}\right)}{\left(P+B_{z}^{2}\right)}
$$

where $P=\frac{\left(4 \psi_{i+1}-3 \psi-\psi_{i+2}\right)}{2 \Delta x}$

Once the values at exterior boundary neighbours have been calculated, updated values can be obtained at all mesh points.

\subsubsection{Axial flux conservation}

The above method of solution relies on the difference equations being a close enough approximation to the differential equations to ensure axial flux conservation. Unfortunately the long time scale nature of the problem causes any small amount flux lost after each time step to eventually build to an unacceptable level. We use two approaches to overcome the problem. 
The first method involves writing the $B_{z}$ evolution equation in a conservative type form as :

$$
\frac{\partial B_{z}}{\partial t}=\frac{\partial^{2} B_{z}}{\partial x^{2}}+\frac{\partial^{2} B_{z}}{\partial y^{2}}+(1-\varepsilon)\left\{\frac{\partial U}{\partial x}+\frac{\partial V}{\partial y}\right\}
$$

where:

$$
U=F \frac{\partial \psi}{\partial x} \quad ; \quad V=F \frac{\partial \psi}{\partial y}
$$

Then using central differences the evolution equation reduces to :

$$
\begin{aligned}
B_{z}{ }^{n+1}= & B_{z}+\Delta t\left\{\frac{\left(B_{z}\right)_{i+1}-\left(B_{z}\right)+\left(B_{z}\right)_{i-1}}{\Delta x^{2}}+\frac{\left(B_{z}\right)_{j+1}-\left(B_{z}\right)+\left(B_{z}\right)_{j-1}}{\Delta y^{2}}\right. \\
& \left.+(1-\varepsilon)\left[\frac{U_{i+1}-U_{i-1}}{2 \Delta x}+\frac{V_{j+1}-V_{j-1}}{2 \Delta y}\right)\right\}
\end{aligned}
$$

Define the flux locally as

$$
\Delta \Phi=\frac{\Delta x \Delta y}{4}\left(\left(B_{z}\right)_{i, j}+\left(B_{z}\right)_{i+1, j}+\left(B_{z}\right)_{i, j+1}+\left(B_{z}\right)_{i+1, j+1}\right)
$$




$$
\Phi=\sum_{i=1}^{n x-1} \sum_{j=1}^{n y-1} \Delta \Phi
$$

where $n x$ and ny are the number of mesh points along $x$ and $y$ respectively.

Summing the $B_{z}$ difference equation as defined by the total axial flux gives an expression \{ Appendix B \} for the flux at the advanced time :

$$
\Phi^{n+1}=\Phi+\frac{\Delta t \Delta x \Delta y}{4}
$$

$$
\left\{\frac{2}{\Delta x^{2}} \sum_{j=1}^{n}-\left(\left(B_{z}\right)_{2}-\left(B_{z}\right)_{0}\right)+\left(B_{z}\right)_{m+1}-\left(B_{z}\right)_{m-1}\right.
$$

$$
+\frac{2}{\Delta y^{2}} \sum_{i=1}^{m-1}-\left(\left(B_{z}\right)_{2}-\left(B_{z}\right)_{0}\right)+\left(B_{z}\right)_{n+1}-\left(B_{z}\right)_{n-1}
$$

$$
\begin{aligned}
& +\frac{(1-\varepsilon)}{\Delta x} \sum_{j=1}^{n-1}-\left(u_{2}+2 u_{1}+\left(1_{0}\right)+\left(u_{m+1}+2\left(u_{m}+\left(u_{m-1}\right)\right.\right.\right. \\
& \left.+\frac{(1-\varepsilon)}{\Delta x} \sum_{i=1}^{m-1}-\left(V_{2}+2 V_{1}+V_{0}\right)+\left(V_{n+1}+2 V_{n}+V_{n-1}\right)\right\}
\end{aligned}
$$

These terms can be interpreted as the excess flux, incurred by discretisation, crossing the boundary as interior to the boundary all excess flux has cancelled between pairs of adjacent mesh points. 
Now consider the solution of the following PDE :

$$
\frac{\partial U}{\partial t}=\frac{\partial^{2} U}{\partial x^{2}}+\frac{\partial U}{\partial x}
$$

satisfying $U(x=0)=U(x=L)=0$ for all $\mathrm{t}$.

The boundary condition can be implemented simply by setting $U$ equal to zero at the interface at each time step.

A second method is to use exterior boundary neighbours. When calculating the first term the exterior value of $U$ is chosen such that the first term vanishes. Similarly for the second term. This approach provides the difference equation with the correct boundary information and takes the form of gradients.

With this in mind $B_{z}$ on the boundary is calculated such that the above terms, arising from summation of the $B_{z}$ difference equation, vanish. The axial flux then remains an invariant of motion.

Here the $B_{z}$ difference equation is being input the correct information, namely that the net flux across the interface is zero, and takes the form of gradients. This is of course consistent with the boundary conditions.

The second method is a much simpler approach than the first and involves a simple scaling. Consider the $B_{z}$ equation in the form

$$
\frac{\partial B_{z}}{\partial t}=\nabla^{2} B_{z}+(1-\varepsilon)\left\{F \nabla^{2} \psi+\frac{\partial \psi}{\partial x} \frac{\partial F}{\partial x}+\frac{\partial \psi}{\partial y} \frac{\partial F}{\partial y}\right\}
$$


At the advanced time the new flux will be slightly different from the preceding time. To obtain the correct flux the values of $B_{z}$ at each mesh point are incremented by the same amount $\beta$ say. If there are $n x$ and $n y$ mesh points along the horizontal and vertical mesh lines respectively then we must have :

$$
\begin{aligned}
\Phi^{n+1} & =\frac{\Delta x \Delta y}{4} \sum_{i=1}^{n x-1} \sum_{j=1}^{n y-1}\left(\left(B_{z}\right)_{i, j}^{n}+\left(B_{z}\right)_{i+1, j}{ }^{n}+\left(B_{z}\right)_{i, j+1}^{n}+\left(B_{z}\right)_{i+1, j+1}{ }^{n}+4 \beta\right) \\
& =\Phi^{n}+\Delta x \Delta y(n x-1)(n y-1) \beta
\end{aligned}
$$

Therefore scaling by :

$$
\beta=\frac{\Phi^{n+1}-\Phi^{n}}{\Delta x \Delta y(n x-1)(n y-1)}
$$

will ensure flux conservation. Since this method scales all mesh points, including the exterior boundary neighbours, the gradients in $B_{2}$ are in no way altered and so the scaling can be considered as a correction to the difference equation incurred by discretisation. Both of the above methods will be implemented.

Solutions to the short time scale evolution equations can now be obtained for differing values of $\alpha$ and a suitable choice of $\varepsilon$. The most desirable value to choose for $\varepsilon$ is the smallest, but non-zero to ensure evolution on the long time scale, that is possible. The accuracy of the numerical algorithm then determines the minimum choice of $\varepsilon$. 
We next present a few simple solutions, equilibrium profiles, in order to compare the various methods discussed so far. Consider a square cross-section represented by $10 \times 10$ mesh-points. The values chosen for $\varepsilon$ and $\alpha$ are $10^{-2}$ and 1.0 respectively.

Figures 2.2 and 2.3 show the $B_{z}$ and $\psi$ profiles when axial flux conservation is achieved using a conservative scheme. Figure 2.2 is the solution obtained when solving a cubic for the exterior boundary neighbour as opposed to the simple method of using forward or backward difference formulae as is represented in figure 2.3. There is of course complete agreement. This then justifies the use of the simple approach over the cubic method. Using the simple method, a relaxed state has been obtained when a simple scaling is used to conserve flux. The profiles are presented in figure 2.4 and they show a slight disagreement. The conservative scheme is then to be preferred over the simple scaling.

\subsubsection{Solution in terms of the components of $B$}

Solutions in $3 \mathrm{D}$ will incorporate all three components of the magnetic field and so it is worth seeking solutions using the components in 2D. Such solutions can then be compared to those obtained using the stream function and if agreement exists the methods involved will hopefully be extendable to $3 \mathrm{D}$.

The short time scale evolution equations to be solved are :

$$
\frac{\partial B_{x}}{\partial t}=\nabla^{2} B_{x}-(1-\varepsilon)\left\{B_{z} \frac{\partial F}{\partial y}+F \frac{\partial B_{z}}{\partial y}\right\}
$$




$$
\begin{aligned}
& \frac{\partial B_{y}}{\partial t}=\nabla^{2} B_{y}-(1-\varepsilon)\left\{B_{z} \frac{\partial F}{\partial x}+F \frac{\partial B_{z}}{\partial x}\right\} \\
& \frac{\partial B_{z}}{\partial t}=\nabla^{2} B_{x}-(1-\varepsilon)\left\{\frac{\partial}{\partial y}\left(F B_{x}\right)-\frac{\partial}{\partial x}\left(F B_{y}\right)\right\}
\end{aligned}
$$

\subsubsection{Boundary conditions}

The boundary conditions written in terms of the components rather than $\psi$ become :

$$
\begin{aligned}
& \frac{\partial B_{z}}{\partial x}=\frac{-\alpha(1-\varepsilon) B_{y} B_{z}}{B_{y}{ }^{2}+B_{z}{ }^{2}} \\
& \frac{\partial B_{y}}{\partial x}=\frac{\alpha\left(\varepsilon B_{y}{ }^{2}+B_{z}{ }^{2}\right)}{B_{y}{ }^{2}+B_{z}{ }^{2}} \\
& B_{x}=0
\end{aligned}
$$

on ad, bc and :

$$
\frac{\partial B_{z}}{\partial y}=\frac{\alpha(1-\varepsilon) B_{x} B_{z}}{B_{x}{ }^{2}+B_{z}{ }^{2}}
$$




$$
\begin{aligned}
& \frac{\partial B_{x}}{\partial y}=\frac{-\alpha\left(\varepsilon B_{x}^{2}+B_{z}^{2}\right)}{B_{x}^{2}+B_{z}^{2}} \\
& B_{y}=0
\end{aligned}
$$

on $a b$ and dc.

These can be implemented by replacing derivatives with central differences giving expressions for the unknown values at exterior boundary neighbours. Axial flux conservation can be achieved as in the stream function case and the only problem is ensuring that $\mathbf{B}$ remains divergence-free.

\subsubsection{Satisfying the divergence-free constraint}

In the analytic solutions of problems involving the divergence-free constraint, the initial state of $\mathbf{B}$ is chosen to be divergence-free and the induction equation (1.3) ensures that $\mathbf{B}$ remains so for subsequent times. The discretisation errors associated with the finite difference method cannot ensure that $\mathbf{B}$ remains divergence-free for all time steps. The constraint is no longer an initial condition and has to be imposed at each time step.

Two methods of ensuring that the constraint remains satisfied are presented.

The first method involves the use $\nabla \cdot \mathbf{B}=0$ itself. Initially $B_{x}, B_{y}$ and $B_{z}$ are known at all mesh points from the initial conditions. At the advanced time step $B_{x}$ and $B_{z}$ are updated using the evolution equations but $B_{y}$ is obtained using $\nabla \cdot \mathbf{B}=0$ in the following way. We want 
$\frac{\partial B_{x}}{\partial x}+\frac{\partial B_{y}}{\partial y}=0 \Rightarrow \frac{\left(B_{x}\right)_{i+1, j}-\left(B_{x}\right)_{i-1, j}}{\Delta x}+\frac{\left(B_{y}\right)_{i, j+1}-\left(B_{y}\right)_{i, j-1}}{\Delta y}=0$

Consider point $\mathrm{p}$ ( figure 2.5 ). At the new time step $B_{x}$ has been calculated at all mesh points and on ab the normal component of $\mathbf{B}, B_{y}$, is zero. Therefore $B_{y}$ at point $\mathrm{q}$ can be calculated using the divergence-free constraint. Once $B_{y}$ at $\mathrm{q}$ is known it can be used, by considering point $\mathrm{p} 2$, to update $B_{y}$ at $\mathrm{q} 2$. Similarly $B_{y}$ at all alternate mesh points can be calculated. This process can then be repeated from dc to ab assuming an even number of mesh points. The updated $B_{y}$ values are then known at all mesh points except the two boundaries ad and bc. These can be obtained using the $B_{y}$ evolution equation.

At the next time step $B_{x}$ is then obtained using $\nabla \cdot \mathbf{B}=0$ and $B_{y}$ and $B_{z}$ are obtained using the evolution equations. If this cycle is then repeated the magnetic field remains divergence-free.

Here one of the difference equations is being compromised in favour of the divergence-free constraint. The process can be considered as a correction to the discretisation error, inherent in the evolution equation, by the divergence-free constraint.

The second and much simpler method uses the symmetry properties of the difference form of mixed derivatives and involves writing the $B_{x}$ and $B_{y}$ evolution equations as :

$$
\frac{\partial B_{x}}{\partial t}=\frac{\partial}{\partial y}\left(\frac{\partial B_{x}}{\partial y}\right)-\frac{\partial^{2} B_{y}}{\partial x \partial y}-(1-\varepsilon) \frac{\partial}{\partial y}\left(F B_{z}\right)
$$




$$
\frac{\partial B_{y}}{\partial t}=\frac{\partial}{\partial x}\left(\frac{\partial B_{y}}{\partial x}\right)-\frac{\partial^{2} B_{x}}{\partial y \partial x}-(1-\varepsilon) \frac{\partial}{\partial x}\left(F B_{z}\right)
$$

We then want to ensure :

$$
\left[\frac{\partial B_{x}}{\partial x}+\frac{\partial B_{y}}{\partial y}\right)=0
$$

in difference form use the following representations

$$
\frac{\partial B}{\partial x} \rightarrow \frac{\left(B_{i+1}-B_{i-1}\right)}{2 \Delta x}
$$

$$
\frac{\partial}{\partial x}\left(\frac{\partial B}{\partial x}\right) \rightarrow \frac{\left(B_{i+2}-2 B_{i}+B_{i-2}\right)}{4 \Delta x^{2}}
$$

$$
\frac{\partial^{2} B}{\partial x \partial y} \rightarrow \frac{\left(B_{i+1, j+1}-B_{i-1, j+1}-B_{i+1, j-1}+B_{i-1, j-1}\right)}{4 \Delta x \Delta y}
$$

It can then be shown $\{$ Appendix D \} that irrespective of discretisation errors $\nabla \cdot \mathbf{B}$ is always zero, provided the evolution equations are used to update values at the four nearest neighbours. This will lead to problems at interior boundary neighbours as $\mathbf{n} \cdot \mathbf{B}$ is used to assign values on the boundary. For the above case, and in general when Cartesian co-ordinates are used, it can be shown ( Appendix D \} that the problem does not arise. 
Both of the above methods have been considered and as before we present a few simple relaxed profiles.

Figures 2.6 and 2.7 show the results obtained when a conservative scheme is used. Figure 2.6 is that obtained when $\nabla \cdot \mathbf{B}=0$ itself is used to satisfy the $\nabla \cdot \mathbf{B}=()$ constraint. They show good agreement with each other and when compared to the $\psi$ case there is complete agreement between figure 2.3 and 2.7. Writing the evolution equations such that $\nabla \cdot \mathbf{B}=0$ is satisfied automatically is then the most accurate way of satisfying the divergence-free constraint. Using this method and achieving flux conservation using a scaling gives the results shown in figure 2.8 . This method also gives very similar results.

All results presented so far have assumed finite currents flow at an interface. When considering a current-free surface, all codes except one become numerically unstable. The only code to acceptable is that incorporating all the components of the magnetic field and where the divergence-free constraint is used to satisfy the constraint itself. This then highlights the importance of considering as many approaches as possible when obtaining numerical solutions.

\subsection{D-Solutions}

Consider the solid to be in the form of a rectangular cylinder of axial length $\mathrm{L}$ and surrounded by a perfectly conducting shell. In 3D there are several ways of driving the system. The simplest is to apply the axial driving field across the full length of the rim. In such a case, the initial state of $\mathbf{B}$ must be chosen to have a $z$ dependence ( non-axisymmetric ). Otherwise the solution at all time steps will be axially independent ( axisymmetric ) and the final solution will always be the same as that obtained in the $2 \mathrm{D}$ case. 
A second and more realistic model would involve the introduction of one or more poloidal gaps at suitable points on the boundary. The driving field would then be applied across these gaps. The variation along the axial length of the boundary then allows the system to seek out non-axisymmetric solutions. A simple axisymmetric $\mathbf{B}$ state can then be chosen as an initial condition.

Both of these models will be considered.

The derivative boundary conditions turn out to be the same as the $2 \mathrm{D}$ case for boundaries normal to the cross section. This is hardly surprising as the geometry of the boundary is independent of $\mathrm{z}$. Along the length of the cylinder periodic boundary conditions will be used :

$$
\mathbf{B}\left(z_{o}-d z\right)=\mathbf{B}(L-d z)
$$

The methods used to ensure $\nabla \cdot \mathbf{B}=0$ in $2 \mathrm{D}$ are directly extendable to $3 \mathrm{D}$.

For the first method the evolution equations are written as :

$$
\begin{aligned}
& \frac{\partial B_{x}}{\partial t}=\nabla^{2} B_{x}-(1-\varepsilon)\left\{B_{z} \frac{\partial F}{\partial y}-B_{y} \frac{\partial F}{\partial z}+F\left(\frac{\partial B_{z}}{\partial y}-\frac{\partial B_{y}}{\partial z}\right)\right\} \\
& \frac{\partial B_{y}}{\partial t}=\nabla^{2} B_{y}-(1-\varepsilon)\left\{B_{x} \frac{\partial F}{\partial z}-B_{z} \frac{\partial F}{\partial x}+F\left(\frac{\partial B_{x}}{\partial z}-\frac{\partial B_{z}}{\partial x}\right)\right\}
\end{aligned}
$$




$$
\begin{aligned}
\frac{\partial B_{z}}{\partial t}= & \frac{\partial^{2} B_{z}}{\partial x^{2}}+\frac{\partial^{2} B_{z}}{\partial y^{2}}-\frac{\partial^{2} B_{x}}{\partial x \partial z}-\frac{\partial^{2} B_{y}}{\partial y \partial z} \\
& -(1-\varepsilon)\left\{\frac{\partial}{\partial x}\left(F B_{y}\right)-\frac{\partial}{\partial y}\left(F B_{x}\right)\right\}
\end{aligned}
$$

The $\nabla \cdot \mathbf{B}=0$ constraint is satisfied using the constraint itself.

It should be noted that the term $\partial^{2} B_{z} / \partial z^{2}$ has been replaced by mixed derivatives, using $\nabla \cdot \mathbf{B}=0$, in the $B_{z}$ evolution equation. This is necessary to ensure axial flux conservation as the term $\partial^{2} B_{z} / \partial z^{2}$ would give contributions to the flux interior to the boundary.

In the second method the evolution equations are written as :

$$
\begin{aligned}
\frac{\partial B_{x}}{\partial t}= & \frac{\partial}{\partial y}\left[\frac{\partial B_{x}}{\partial y}\right]+\frac{\partial}{\partial z}\left[\frac{\partial B_{x}}{\partial z}\right]-\frac{\partial^{2} B_{y}}{\partial x \partial y}-\frac{\partial^{2} B_{z}}{\partial x \partial z} \\
& -(1-\varepsilon)\left\{\frac{\partial}{\partial y}\left(F B_{z}\right)-\frac{\partial}{\partial z}\left(F B_{y}\right)\right\} \\
\frac{\partial B_{y}}{\partial t}= & \frac{\partial}{\partial x}\left\{\frac{\partial B_{y}}{\partial x}\right\}+\frac{\partial}{\partial z}\left[\frac{\partial B_{y}}{\partial z}\right)-\frac{\partial^{2} B_{x}}{\partial x \partial y}-\frac{\partial^{2} B_{z}}{\partial y \partial z} \\
& -(1-\varepsilon)\left\{\frac{\partial}{\partial z}\left(F B_{x}\right)-\frac{\partial}{\partial x}\left(F B_{z}\right)\right\}
\end{aligned}
$$




$$
\begin{aligned}
\frac{\partial B_{z}}{\partial t}= & \frac{\partial}{\partial x}\left[\frac{\partial B_{z}}{\partial x}\right]+\frac{\partial}{\partial y}\left[\frac{\partial B_{z}}{\partial y}\right]-\frac{\partial^{2} B_{x}}{\partial x \partial z}-\frac{\partial^{2} B_{y}}{\partial y \partial z} \\
& -(1-\varepsilon)\left\{\frac{\partial}{\partial x}\left(F B_{y}\right)-\frac{\partial}{\partial y}\left(F B_{x}\right)\right\}
\end{aligned}
$$

If the difference representations are chosen as in the $2 \mathrm{D}$ case then again it can be shown $\{$ Appendix D $\}$ that irrespective of discretisation errors $\nabla \cdot \mathbf{B}=0$ is always satisfied. 

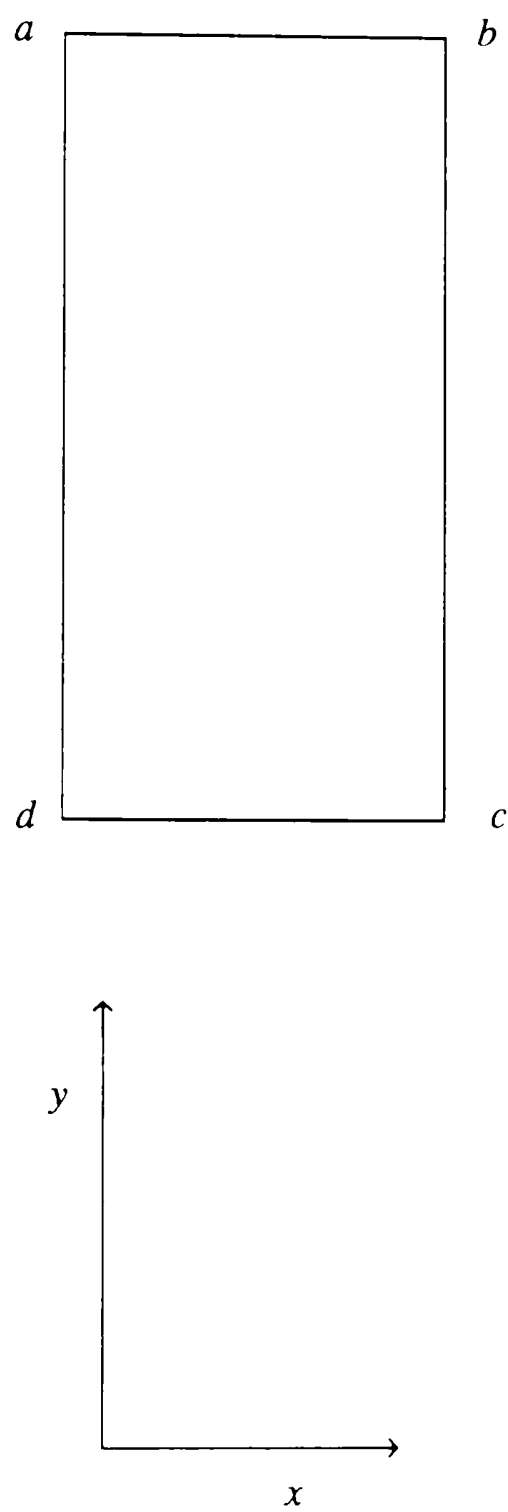

Figure 2.1

Co-ordinate system used when the rectangular region abcd is discretised. 


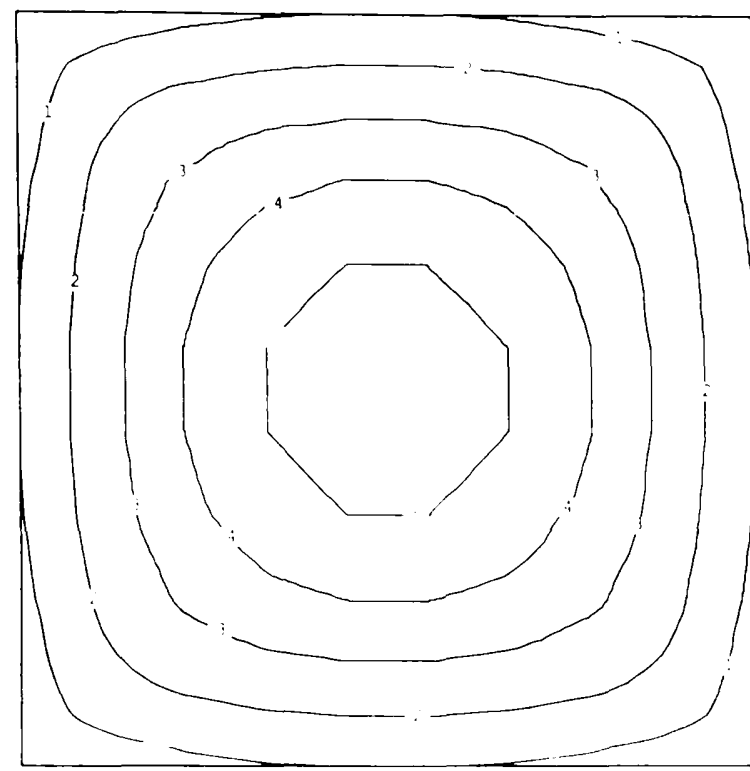

$\mathbf{B}_{\mathbf{z}}$

\begin{tabular}{cc} 
CON TOUR KEY \\
\hline 1 & 0.14 \\
2 & 0.17 \\
3 & 0.20 \\
4 & 0.24 \\
5 & 0.27 \\
\hline
\end{tabular}

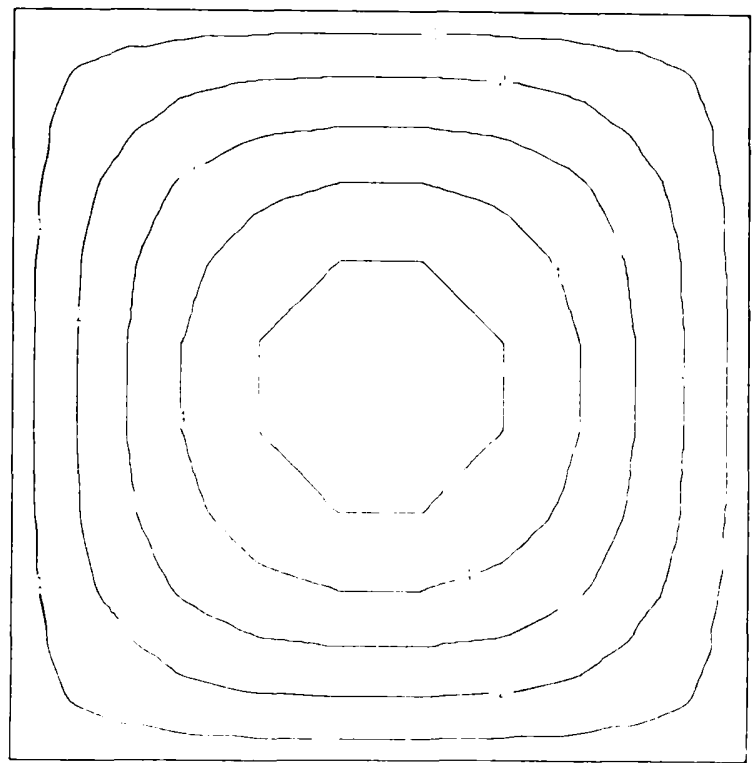

$\psi$

CONTOUR KEY

2.21

0.12

$\ddot{3} .13$

6.14

0.15

Figure 2.2

Contours of $B_{z}$ and $\psi$ when axial flux is conserved using a conservative scheme. Exterior boundary neighbours were obtained by using forward or backward difference formulae. 

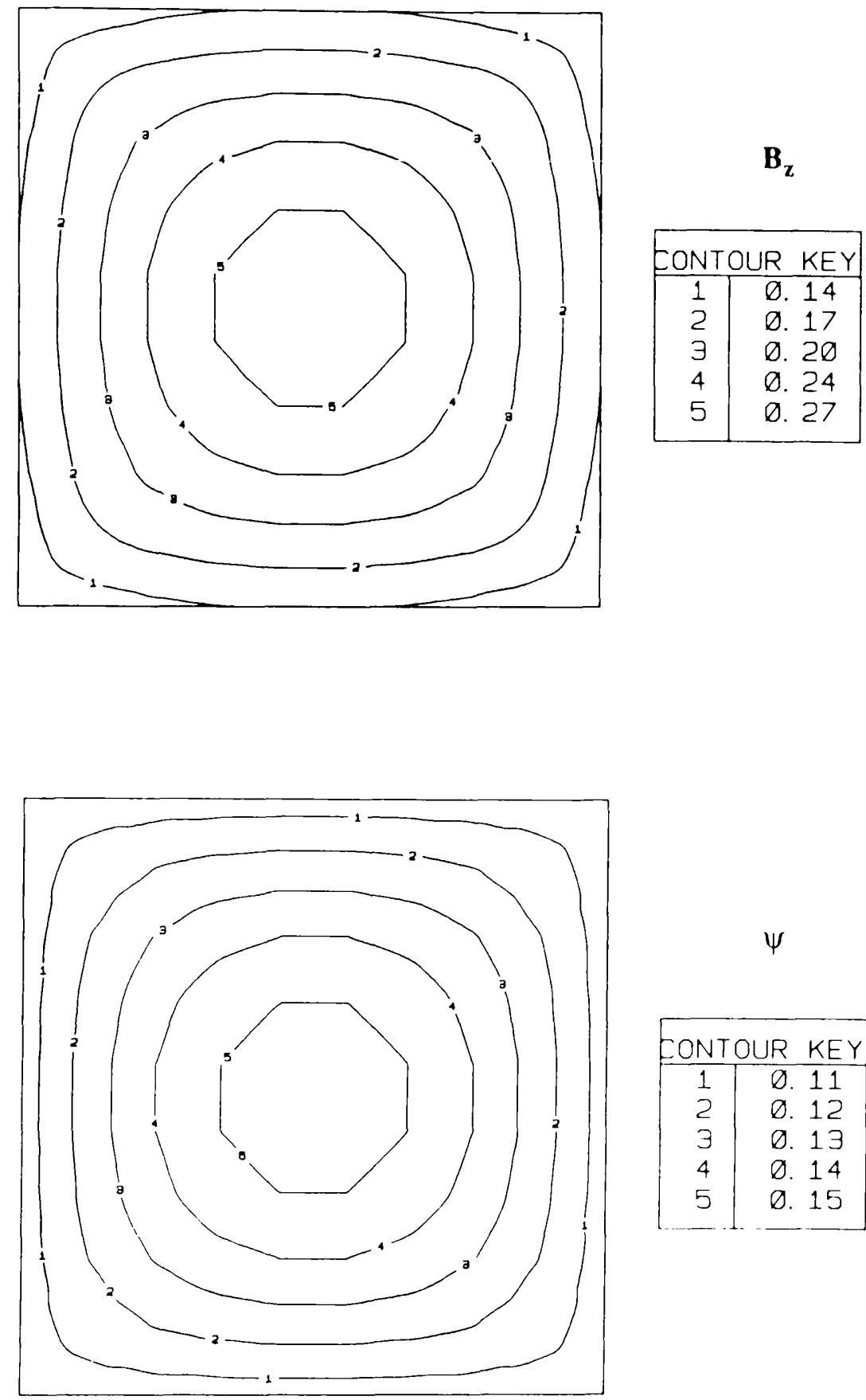

$\psi$

\begin{tabular}{|c|c|c|}
\hline EONTOUR & KEY \\
\hline 1 & 0.11 \\
2 & 0.12 \\
3 & 0.13 \\
4 & $\emptyset .14$ \\
5 & $\emptyset .15$ \\
\hline
\end{tabular}

Figure 2.3

Contours of $B_{z}$ and $\psi$ when axial flux is conserved using a conservative scheme. Exterior boundary neighbours were obtained by solving a cubic. 


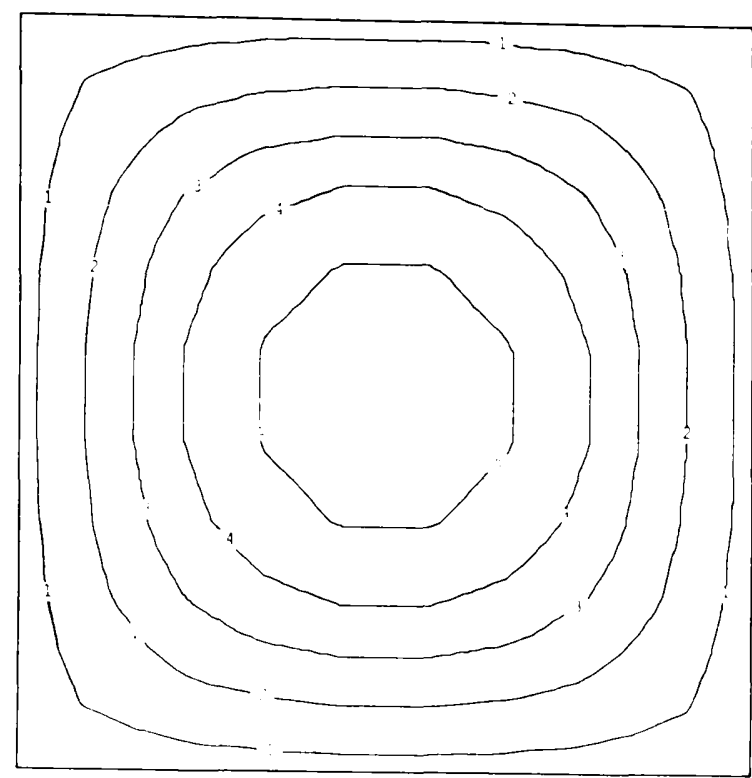

\begin{tabular}{cc}
\multicolumn{1}{c}{$\mathbf{B}_{\mathbf{z}}$} \\
\\
CONTOUR \\
\hline 2 & KEY \\
\hline 2 & 0.11 \\
3 & 0.21 \\
4 & 0.27 \\
5 & 0.32 \\
\hline
\end{tabular}
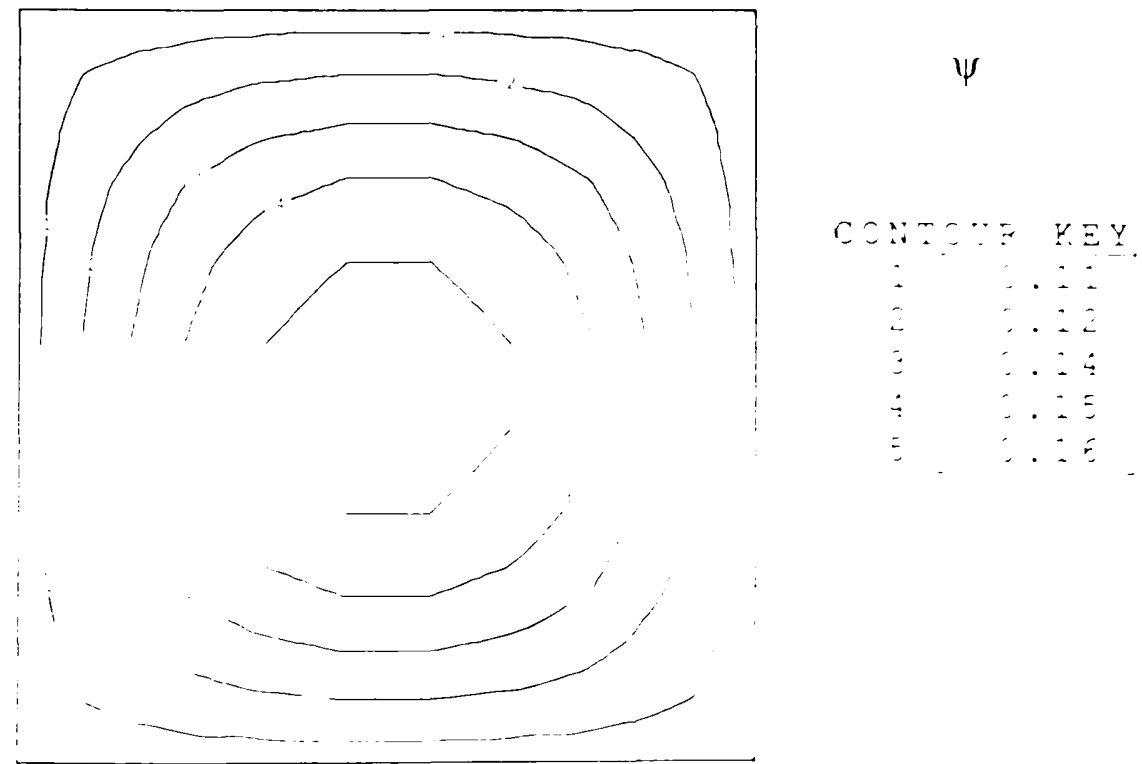

Figure 2.4

Contours of $B_{z}$ and $\psi$ when axial flux is conserved by a simple scaling of $B_{z}$. 


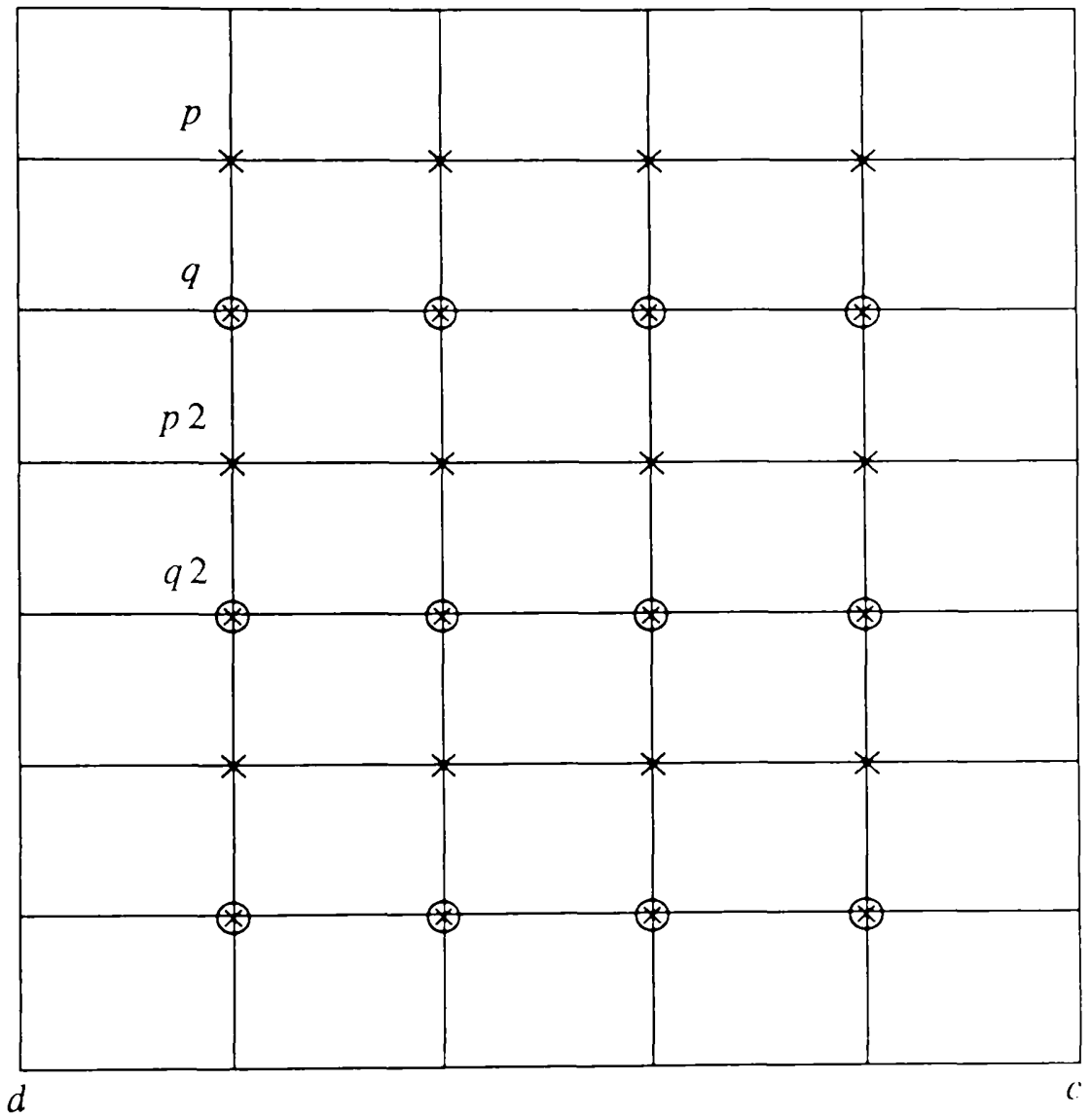

Figure 2.5

The divergence-free constraint is used to update $B_{y}$ at the alternate mesh points $q$ since $B_{y}$ on $a b$ is zero. The process is repeated from $d c$ to $a b$. The value of $B_{y}$ is then known at all mesh points except along $a d$ and $b c$ which are obtaincd using the $B_{y}$ evolution equation. 
Contours of $B_{z}, B_{x}$ and $B_{y}$ when the divergence-free constraint is used to satisfy the constraint itself. Flux is conserved using a conservative scheme. 


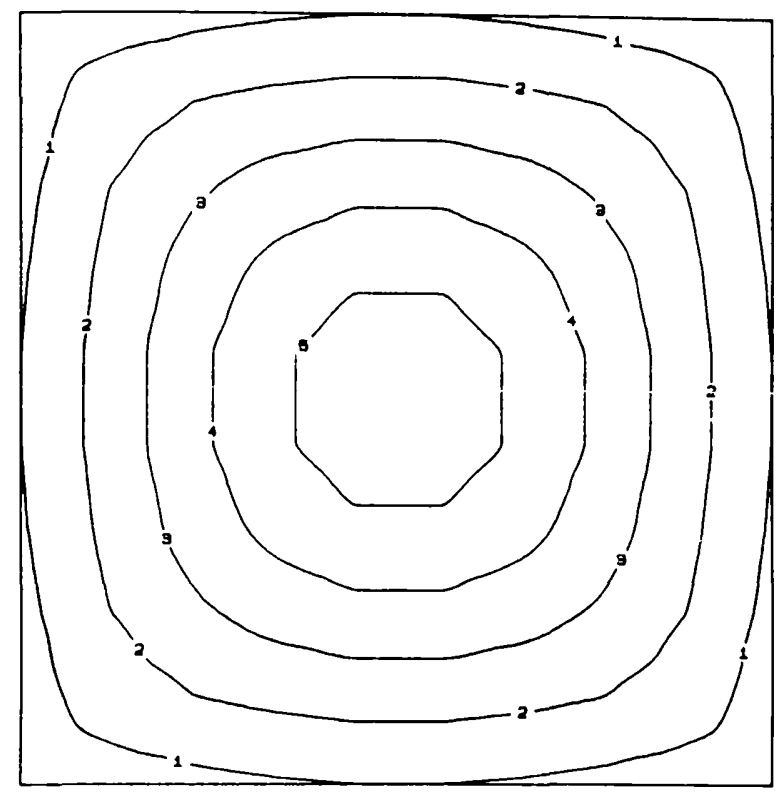

B $_{\mathbf{z}}$

\begin{tabular}{|c|c|}
\hline \multicolumn{2}{|c|}{ EONTOUR } \\
\hline \hline 1 & KEY \\
\hline 2 & 0.14 \\
3 & 0.21 \\
4 & 0.25 \\
5 & 0.29 \\
\hline
\end{tabular}

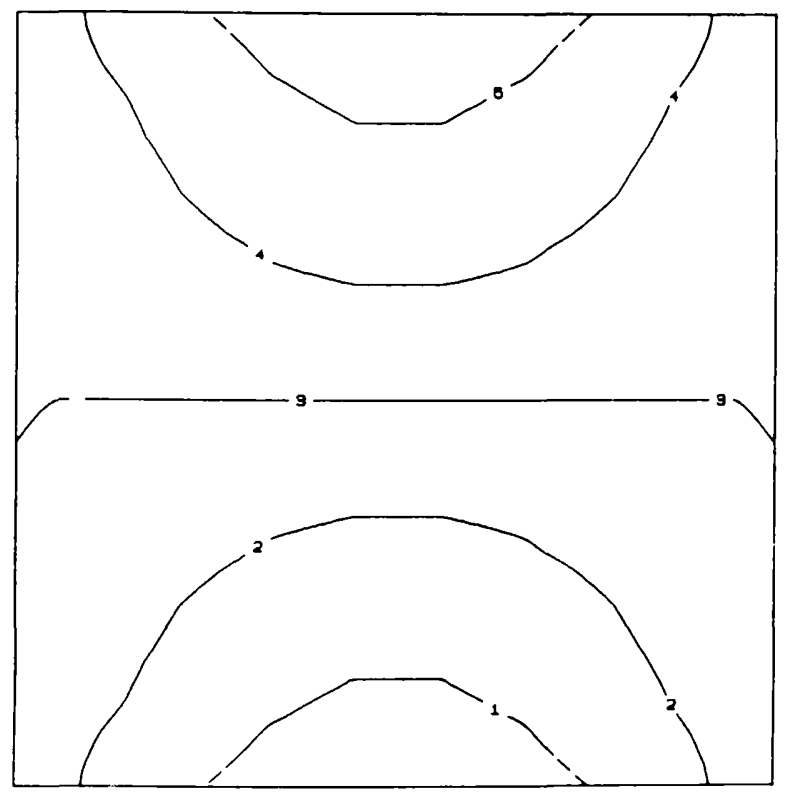

\begin{tabular}{|c|c|}
\hline \multicolumn{2}{|c|}{ EONTOUR KEY } \\
\hline 1 & -0.17 \\
\hline 2 & $-\varnothing . \varnothing 9$ \\
\hline 3 & $\oslash . \oslash 0$ \\
\hline 4 & 0.09 \\
\hline 5 & Ø. 17 \\
\hline
\end{tabular}
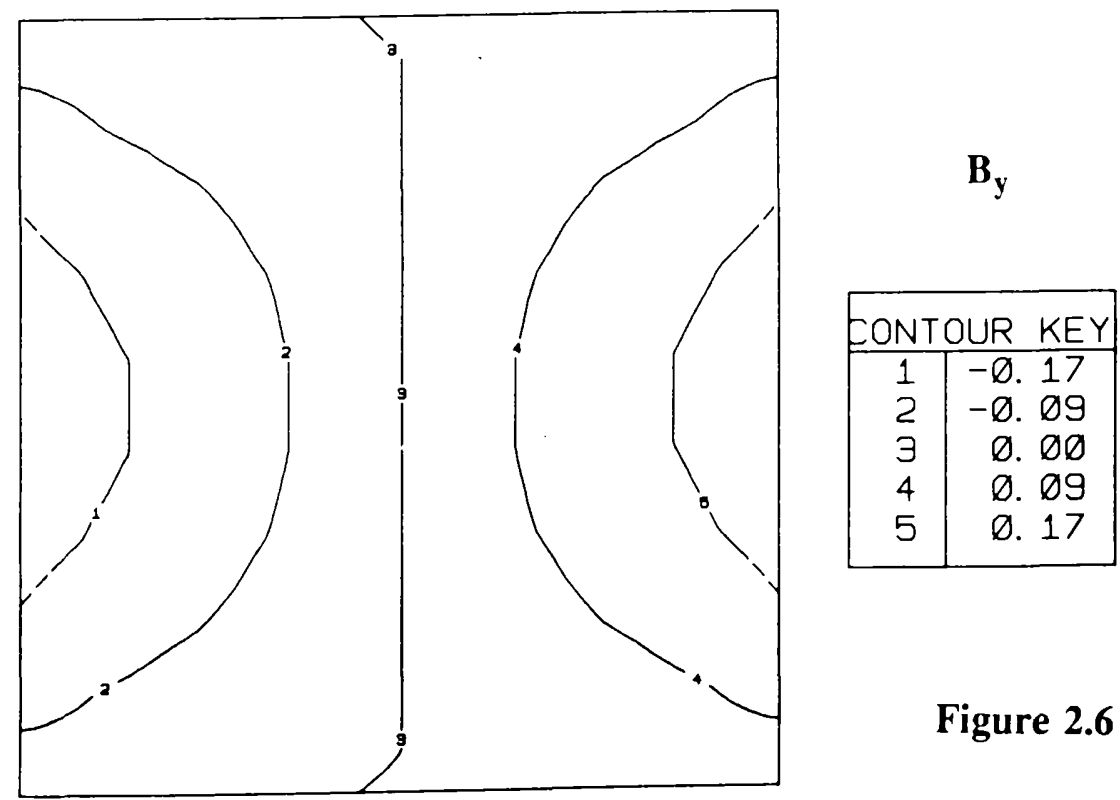

Figure 2.6 
Contours of $B_{z}, B_{x}$ and $B_{y}$ when the divergence-free constraint is satisfied automatically. Flux is conserved using a conservative scheme. 


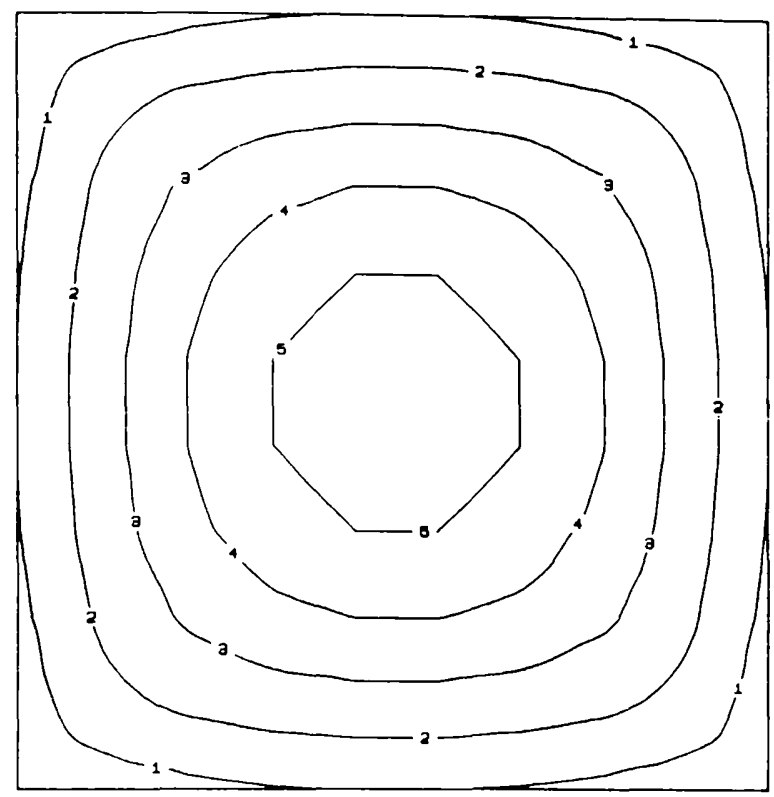

$\mathbf{B}_{\mathrm{z}}$

\begin{tabular}{|c|c|}
\hline \multicolumn{2}{|c|}{ EONTOUR } \\
\hline \hline 1 & KEY \\
\hline 2 & 0.14 \\
3 & 0.17 \\
4 & 0.20 \\
5 & 0.27 \\
\hline
\end{tabular}

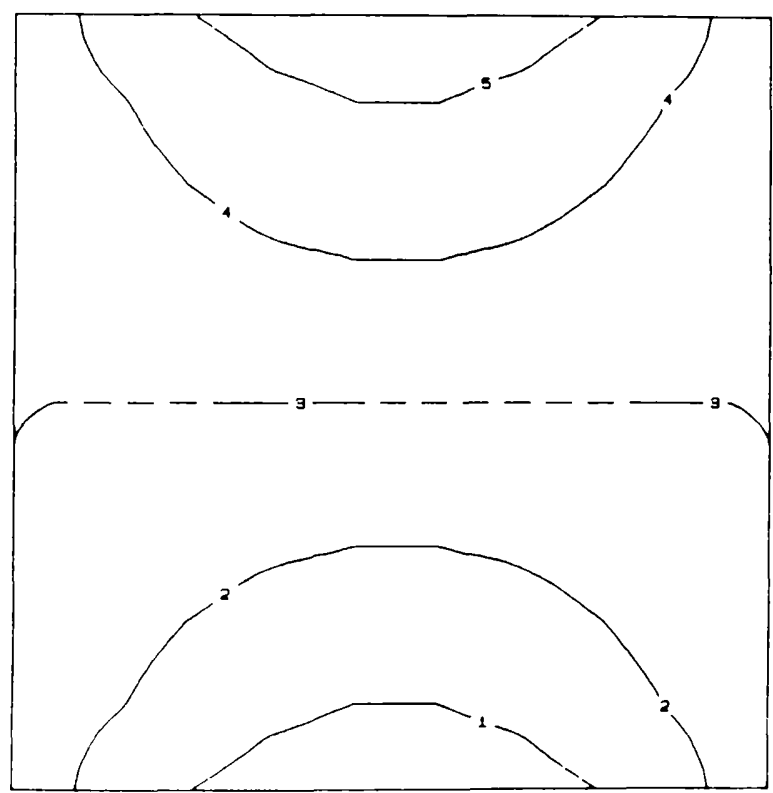

$\mathbf{B}_{\mathbf{x}}$

\begin{tabular}{|c|c|}
\hline \multicolumn{2}{|c|}{ EONTOUR } \\
\hline \multicolumn{2}{|c|}{ KEY } \\
\hline 1 & $-\oslash .16$ \\
2 & $-\oslash . \oslash \theta$ \\
3 & $\emptyset . \oslash \emptyset$ \\
4 & $\oslash . \oslash \theta$ \\
5 & $\emptyset .16$ \\
\hline
\end{tabular}

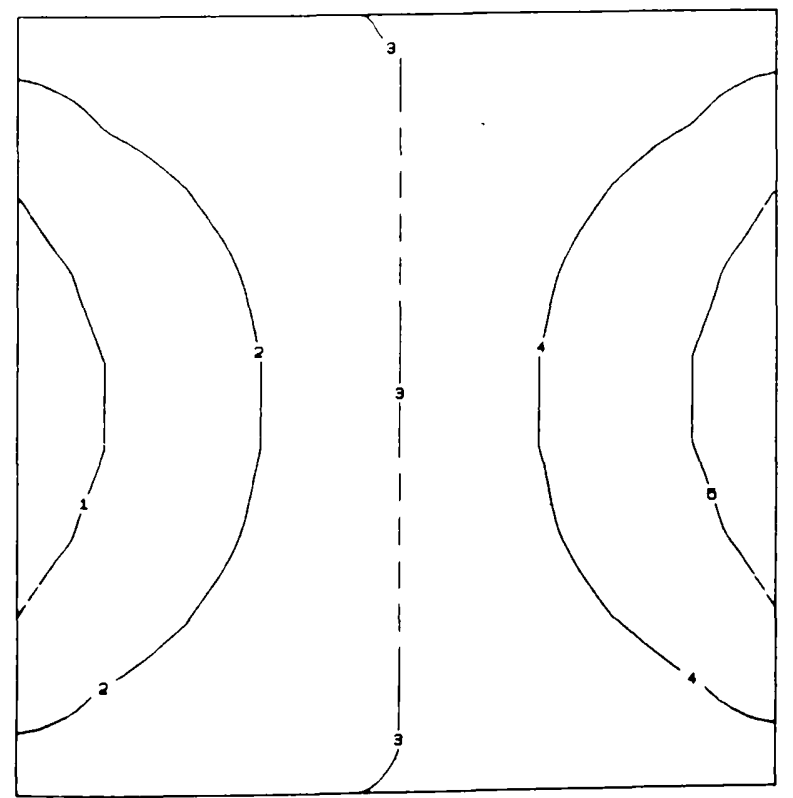

By

EONTOUR KEY

\begin{tabular}{|l|r|}
\hline 1 & $-\emptyset .16$ \\
2 & $-\emptyset . \oslash \theta$ \\
3 & $\emptyset . \emptyset \emptyset$ \\
4 & $\emptyset . \emptyset \theta$ \\
5 & $\emptyset .16$
\end{tabular}

Figure 2.7 
Contours of $B_{z}, B_{x}$ and $B_{y}$ when the divergence-free constraint is satisfied automatically. Flux is conserved by a simple scaling of $B_{\mathbf{z}}$. 


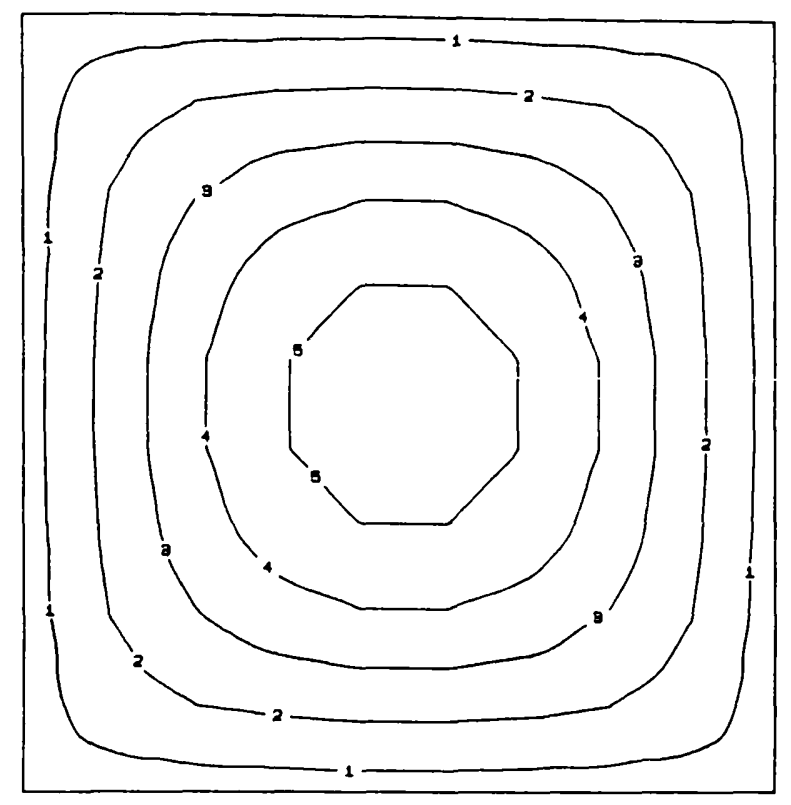

\begin{tabular}{|c|c|}
\hline \multicolumn{2}{|c|}{ EONTOLIR KEY } \\
\hline 1 & 0.15 \\
\hline 2 & Ø. 18 \\
\hline$\exists$ & 0.21 \\
\hline 4 & Ø. 24 \\
\hline 5 & Ø. 27 \\
\hline
\end{tabular}

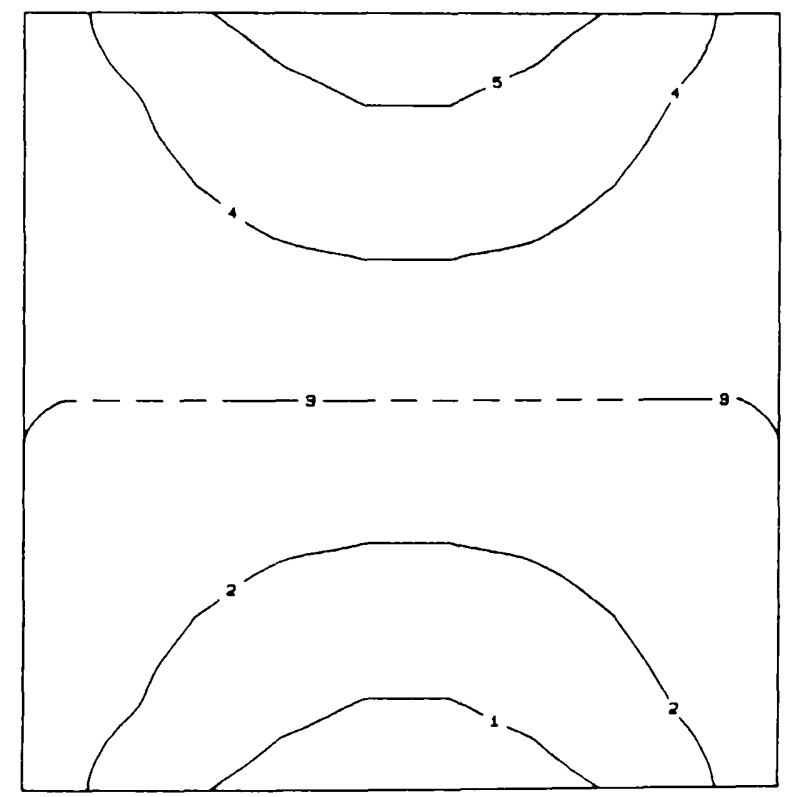

\begin{tabular}{|c|c|}
\hline & $\mathbf{B}_{\mathbf{x}}$ \\
\hline \multicolumn{2}{|c|}{ EONTOUR KEY } \\
\hline 1 & -0.16 \\
\hline 2 & $-\varnothing . \bar{\theta}$ \\
\hline 3 & $\oslash . \oslash \emptyset$ \\
\hline 4 & $\oslash . \oslash \theta$ \\
\hline 5 & Ø. 16 \\
\hline
\end{tabular}
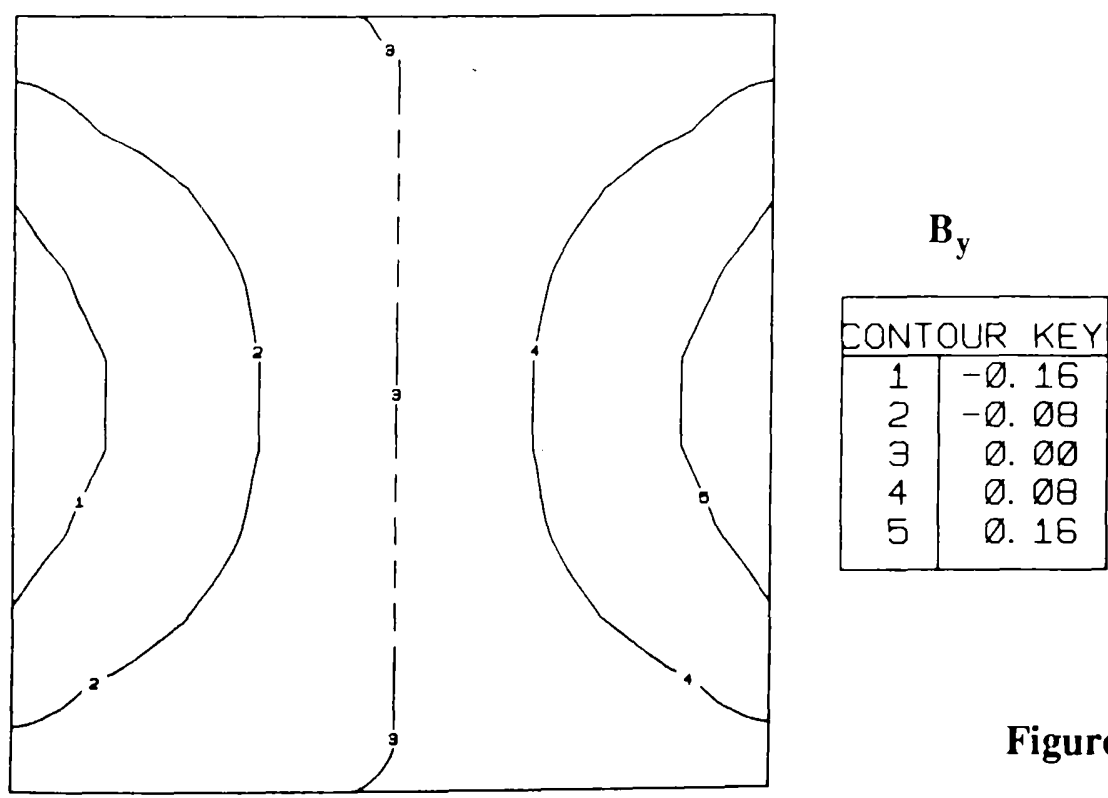

Figure 2.8 


\section{Chapter 3}

\section{D-Curved Boundaries}

All existing plasma containment devices possess a non-rectangular cross-section. These include the simple circle, the D-shape and the more elaborate multipinch. So it is essential to develop a code which will obtain solutions across an arbitrary cross-section.

There are two approaches to finding solutions to PDE's when arbitrary curved boundaries are involved. One approach is to use a co-ordinate system in which the co-ordinate curves conform to the boundary. In this case the difference formulae interior to the boundary can be immensely complicated and may give rise to singularity problems but Neumann type boundary conditions are very simple to implement. The second approach is to use Cartesians which ensure that the difference formulae interior to the boundary remain relatively simple but the boundary conditions are much more difficult to implement. The latter approach will be used here.

In what follows all the components of the magnetic field will be used and solutions using the stream function will not be considered. This is because going into $3 \mathrm{D}$ will involve all the components of $\mathbf{B}$ and so it will merely be a problem of extending the $2 \mathrm{D}$ Code. As in the rectangular boundary case the solutions are assumed to be independent in the $\mathrm{z}$ co-ordinate. 


\subsection{Boundary conditions}

Consider the solid to be in the form of an infinitely long cylinder possessing a non-rectangular cross-section and surrounded by a perfectly conducting shell ( figure 3.1 ). The system is driven as previously and the boundary conditions are obtained as before i.e. by demanding that $\mathbf{n} \cdot \mathbf{B}$ and $\mathbf{n} \times \mathbf{E}$ vanish at the boundary.

Consider figure 3.1. At the interface :

$$
\begin{aligned}
& \mathbf{B}_{n}=0 \\
& \mathbf{E}_{t}=0 \\
& E_{z}=\text { CONSTANT }=C
\end{aligned}
$$

or :

$$
\begin{aligned}
& B_{x} \cos \beta+B_{y} \sin \beta=0 \\
& E_{x} \sin \beta-E_{y} \cos \beta=0 \\
& E_{z}=C
\end{aligned}
$$

After substituting for the components of $\mathbf{E}$, using Ohm's Law, the following conditions \{ Appendix C \} are obtained

$$
\frac{\partial B_{z}}{\partial x}+T \frac{\partial B_{z}}{\partial y}=\frac{\alpha B_{y} B_{z}(\varepsilon-1)\left(1+T^{2}\right)}{B_{y}{ }^{2}\left(1+T^{2}\right)+B_{z}^{2}}
$$




$$
\begin{aligned}
& \frac{\partial B_{y}}{\partial x}-\frac{\partial B_{x}}{\partial y}=\frac{\alpha\left(\varepsilon B_{y}^{2}\left(1+T^{2}\right)+B_{z}^{2}\right)}{B_{y}^{2}\left(1+T^{2}\right)+B_{z}^{2}} \\
& B_{x}+T B_{y}=0
\end{aligned}
$$

where $T=\tan \beta$

The first condition expresses the normal derivative of $B_{z}$ and the second takes the form of the normal derivative of the tangential component.

\subsection{Implementation of the boundary conditions}

In the case of curved boundaries then, the derivatives of $B_{x}$ and $B_{y}$ at the interface are coupled and the method of using exterior boundary neighbours becomes immensely difficult as this introduces two unknowns. One way of overcoming such a problem involves taking forward or backward derivatives into the solid with respect to the boundary. This then gives difference formulae in terms of the unknown boundary values and the known values interior to the boundary which can be obtained using the evolution equations.

Consider point $\mathrm{p}$ ( figure 3.2 ). The derivatives, at $\mathrm{p}$, can be replaced by second order difference formulae which can be written in terms of values on and interior to the boundary, as :

$$
\frac{\partial U}{\partial x}=\frac{h_{1}^{2}\left(U_{R 1}-U_{P}\right)-h_{2}^{2}\left(U_{Q 1}-U_{P}\right)}{h_{1} h_{2}\left(h_{1}-h_{2}\right)}
$$




$$
\frac{\partial U}{\partial y}=\frac{g_{1}^{2}\left(U_{R 2}-U_{P}\right)-g_{2}^{2}\left(U_{Q 2}-U_{P}\right)}{g_{1} g_{2}\left(g_{1}-g_{2}\right)}
$$

Making such replacements in (3.1) and (3.2) and substituting for $B_{x}$ from (3.3) leads to :

$$
\begin{aligned}
& p B_{z}+q=\frac{\alpha B_{y} B_{z}(\varepsilon-1)\left(1+T^{2}\right)}{B_{y}{ }^{2}\left(1+T^{2}\right)+B_{z}{ }^{2}} \\
& a B_{y}+b=\frac{\alpha\left(\varepsilon B_{y}{ }^{2}\left(1+T^{2}\right)+B_{z}{ }^{2}\right)}{B_{y}{ }^{2}\left(1+T^{2}\right)+B_{z}{ }^{2}}
\end{aligned}
$$

where $B_{z}$ and $B_{y}$ are the unknown boundary values and

$$
\begin{aligned}
& a=\left(h_{1}-h_{2}\right)\left(g_{1}+g_{2}\right)\left[g_{1} g_{2}\left(h_{1}+h_{2}\right)+h_{1} h_{2} T\left(g_{2}-g_{1}\right)\right] \div D E N \\
& b=\left[g_{1} g_{2}\left(g_{1}-g_{2}\right)\left(h_{1}^{2} Y_{R 1}-h_{2}^{2} Y_{Q 1}\right)\right. \\
& \left.+h_{1} h_{2}\left(h_{1}-h_{2}\right)\left(g_{2}^{2} X_{Q 2}-g_{1}^{2} X_{R 2}\right)\right] \div D E N \\
& p=a
\end{aligned}
$$$$
q=\left[g_{1} g_{2}\left(g_{1}-g_{2}\right)\left(h_{1}^{2} Z_{R 1}-h_{2}^{2} Z_{Q 1}\right)\right.
$$ 
$\left.+h_{1} h_{2} T\left(h_{1}-h_{2}\right)\left(g_{1}^{2} Z_{R 2}-g_{2}^{2} Z_{Q 2}\right)\right] \div D E N$

$$
D E N=h_{1} h_{2} g_{1} g_{2}\left(h_{1}-h_{2}\right)\left(g_{1}-g_{2}\right)
$$

Now set $B_{y}=m B_{z}$ to give

$$
\begin{aligned}
& p B_{z}+q=\alpha \frac{m(\varepsilon-1)\left(1+T^{2}\right)}{\left(1+m^{2}\left(1+T^{2}\right)\right)} \\
& a m B_{z}+b=\alpha \frac{\left(1+\varepsilon m^{2}\left(1+T^{2}\right)\right)}{\left(1+m^{2}\left(1+T^{2}\right)\right)}
\end{aligned}
$$

Eliminating $B_{2}$ results in the remarkably simple expression

$$
m=\frac{b-\alpha}{q}
$$

This solution of $m$ then gives the components of $\mathbf{B}$ on the boundary. Such a choice will ensure that the boundary conditions are satisfied at each time-step. 


\subsection{Axial flux conservation}

For the rectangular cross section case, the $B z$ difference equation was written such that its sum, as defined by the total axial flux, produced a zero excess flux interior to the boundary. The summation was straight forward along the boundaries and simple expressions for the excess flux crossing the interface were obtained. In the case of curved boundaries the problem is made difficult due to the varying summation limits and a summation along the boundary is in general impossible. The following is a generalisation of the method used for the rectangular case to arbitrary cross-sections.

Consider the solution of the following PDE :

$$
\frac{\partial u}{\partial t}=g
$$

given the following global constraint :

$$
\int_{s} u d s=\text { CONSTANT }
$$

Then referring to figure 3.3 :

$$
\begin{aligned}
& u_{i, j}^{n+1}=u_{i, j}^{n}+d t g_{i, j}^{n} \\
& u_{b}^{n+1}=u_{b}^{n}+d t g_{b}^{n}
\end{aligned}
$$


The flux for the elements of area can be written as :

$$
\begin{aligned}
& \Phi_{l, m}^{n+1}=\frac{1}{4} A_{k}\left(u_{b}^{n+1}+u_{i, j}^{n+1}+u_{b+1}^{n+1}+u_{i, j+1}^{n+1}\right) \\
& \Phi_{l+1, m}^{n+1}=\frac{1}{4} A\left(u_{i, j}^{n+1}+u_{i+1, j}^{n+1}+u_{i, j+1}^{n+1}+u_{i+1, j+1}^{n+1}\right) \\
& \Phi_{l, m+1}^{n+1}=\frac{1}{4} A_{k+1}\left(u_{b+1}^{n+1}+u_{i, j+1}^{n+1}+u_{b+2}^{n+1}+u_{i, j+2}^{n+1}\right) \\
& \Phi_{l+1, m+1}^{n+1}=\frac{1}{4} A\left(u_{i, j+1}^{n+1}+u_{i+1, j+1}^{n+1}+u_{i, j+2}^{n+1}+u_{i+1, j+2}^{n+1}\right)
\end{aligned}
$$

Substituting for the advanced time step values gives :

$$
\begin{aligned}
& \Phi_{l, m}^{n+1}=\Phi_{l, m}^{n}+\frac{1}{4} A_{k} d t\left(g_{b}^{n}+g_{i, j}^{n}+g_{b+1}^{n}+g_{i, j+1}^{n}\right) \\
& \Phi_{l+1, m}^{n+1}=\Phi_{l+1, m}^{n}+\frac{1}{4} A d t\left(g_{i, j+1}^{n}+g_{i, j+1}^{n}+g_{i, j+1}^{n}+g_{i+1, j+1}^{n}\right) \\
& \Phi_{l, m+1}^{n+1}=\Phi_{l, m+1}^{n}+\frac{1}{4} A_{k} d t\left(g_{b+1}^{n}+g_{i, j+1}^{n}+g_{b+2}^{n}+g_{i, j+2}^{n}\right) \\
& \Phi_{l+1, m+1}^{n+1}=\Phi_{l+1, m+1}^{n}+\frac{1}{4} A d t\left(g_{i, j+1}^{n}+g_{i+1, j+1}^{n}+g_{i, j+2}^{n}+g_{i+1, j+2}^{n}\right)
\end{aligned}
$$


Now sum these four fluxes and consider only terms arising along the $b_{k+1}$ horizontal mesh line.

$$
\begin{gathered}
\Phi_{4}^{n+1}=\Phi_{4}^{n}+\frac{1}{4} A_{k} d t\left(g_{b+1}^{n}+g_{i, j+1}^{n}\right)+\frac{1}{4} A d t\left(g_{i, j+1}^{n}+g_{i+1, j+1}^{n}\right)+ \\
\frac{1}{4} A_{k+1} d t\left(g_{b+1}^{n}+g_{i, j+1}^{n}\right)+\frac{1}{4} A d t\left(g_{i, j+1}^{n}+g_{i+1, j+1}^{n}\right)
\end{gathered}
$$

Suppose the net flux after summation over points interior to the interior boundary neighbours along the mesh line being considered is :

$$
\frac{1}{4} d t A f^{n}
$$

The total excess flux obtained for the LHS of the boundary at the advanced time step will then be :

$$
A f^{n}+2 A\left(g_{i, j+1}^{n}+g_{i+1, j+1}^{n}\right)+\left(A_{k}+A_{k+1}\right)\left(g_{b+1}+g_{i, j+1}\right)
$$

If this is set equal to zero, similarly for the RHS, the axial flux will remain an invariant of motion.

Referring to figure 3.4 the above term can be written as :

$$
2 A\left(f+g_{1}+g_{2}\right)+\left(A_{1}+A_{0}\right)\left(g_{1}+g_{b}\right)
$$


Substituting for $g_{b}$ into the evolution equation then gives the updated boundary value :

$$
u_{b}^{n+1}=u_{b}^{n}-\frac{\Delta t}{A_{1}+A_{0}}\left(g_{1}^{n}\left(A_{1}+A_{0}+2 A\right)+2 A\left(f^{n}+g_{2}^{n}\right)\right)
$$

choosing such a value will ensure axial flux conservation.

The divergence-free constraint can be satisfied as in the previous chapter.

A code incorporating the above methods can be used to find solutions over a variety of well known cross-sections. 


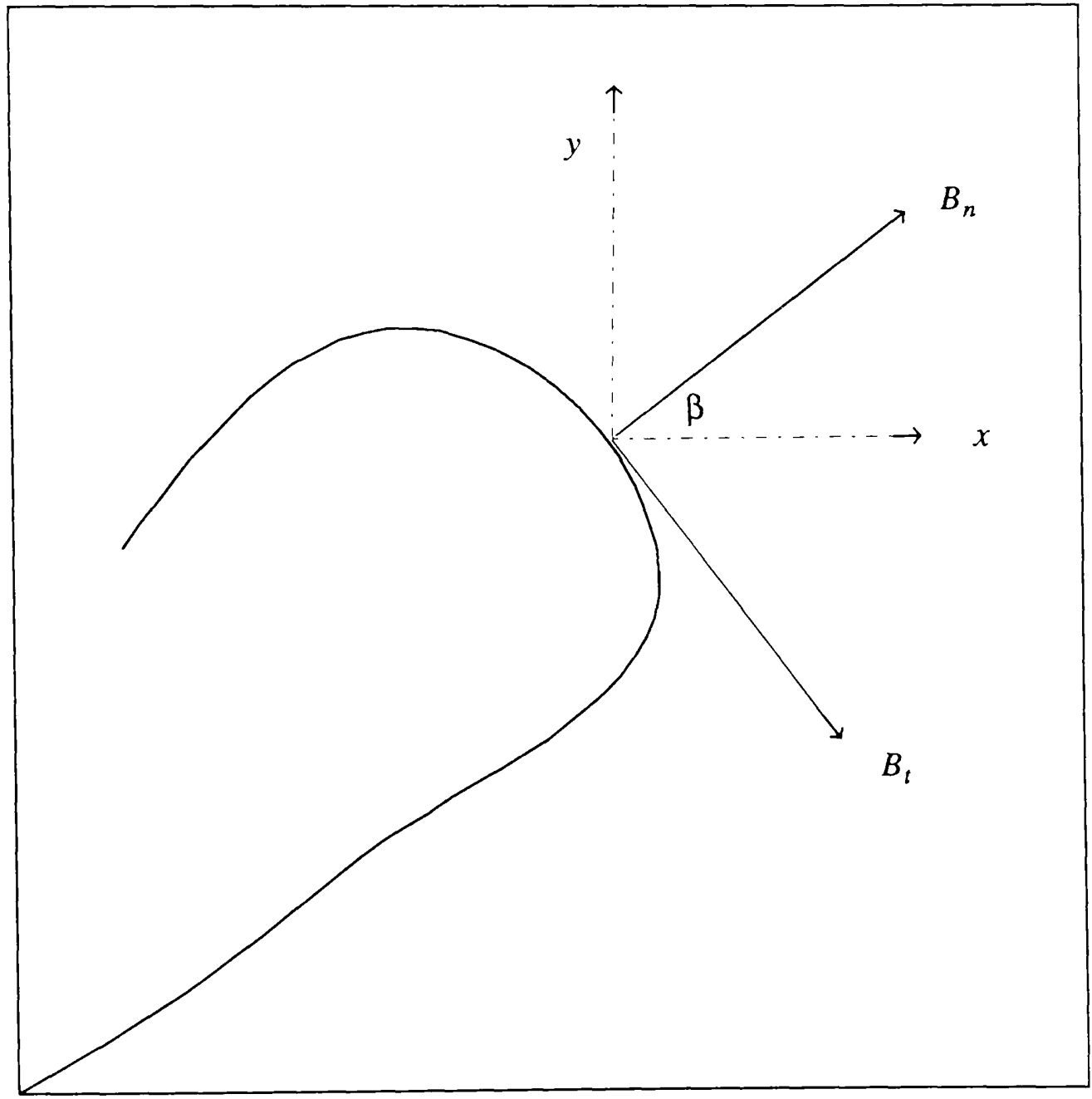

Figure 3.1

Section of arbitrary boundary showing how the direction of the normal and tangential components of $\mathbf{B}$ are defined. 


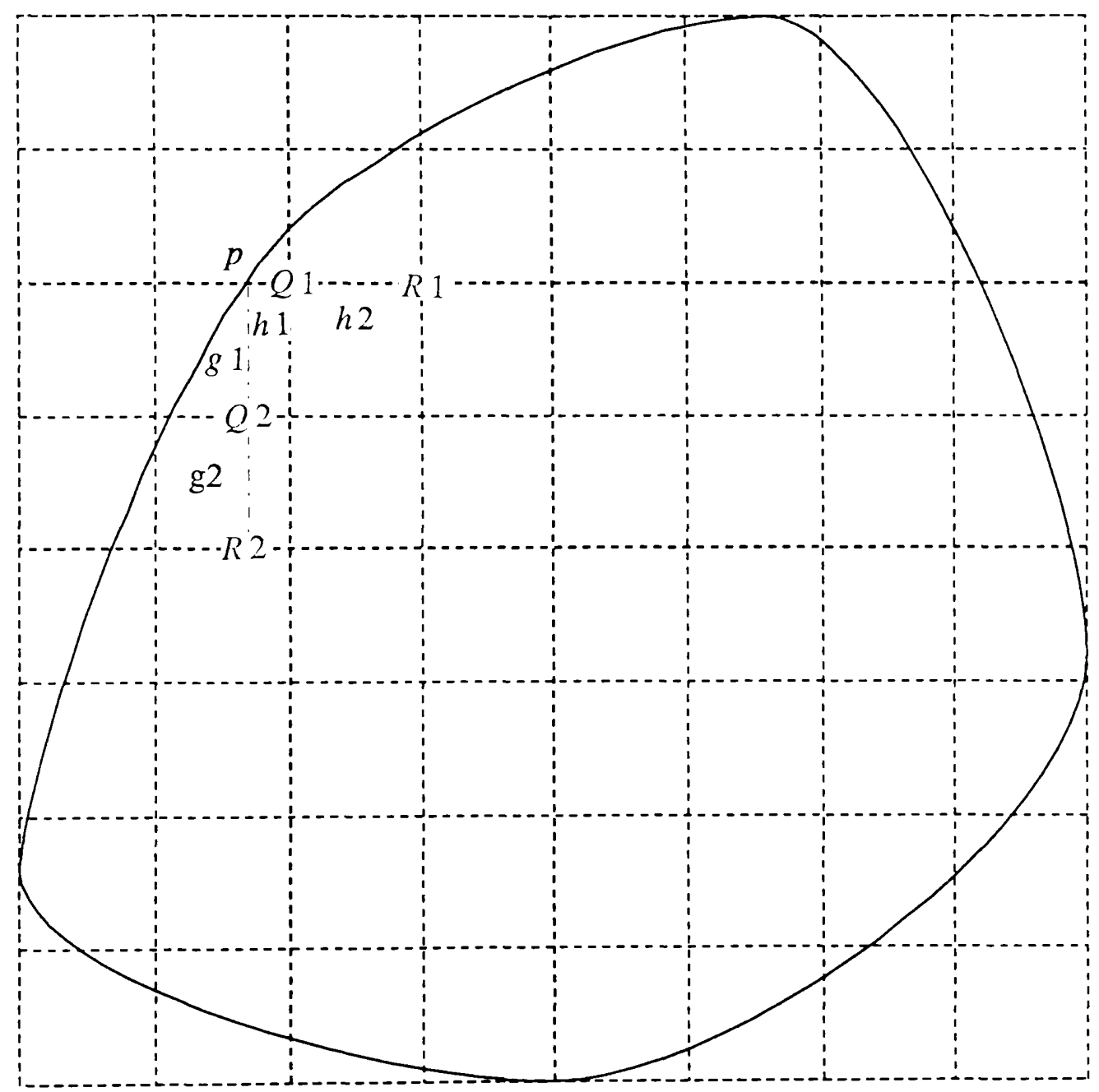

Figure 3.2

Derivatives at point $p$ are replaced by difference formulae involving values on and interior to the boundary. This is useful when the boundary conditions couple the derivatives of $B$ at the interface as it involves only one unknown-the boundary value. 


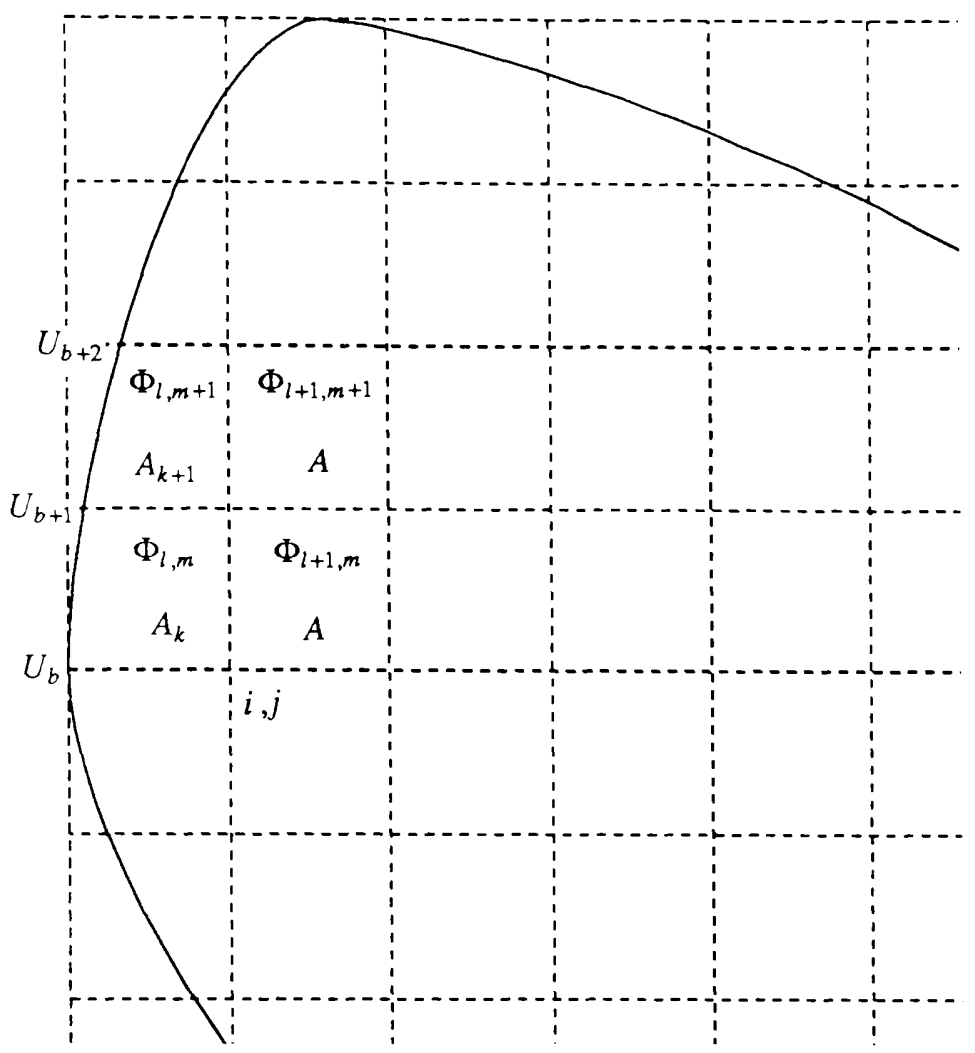

Figure 3.3

The conservative scheme used to ensure axial flux conservation in the rectangular case is generalised to arbitrary cross-sections.

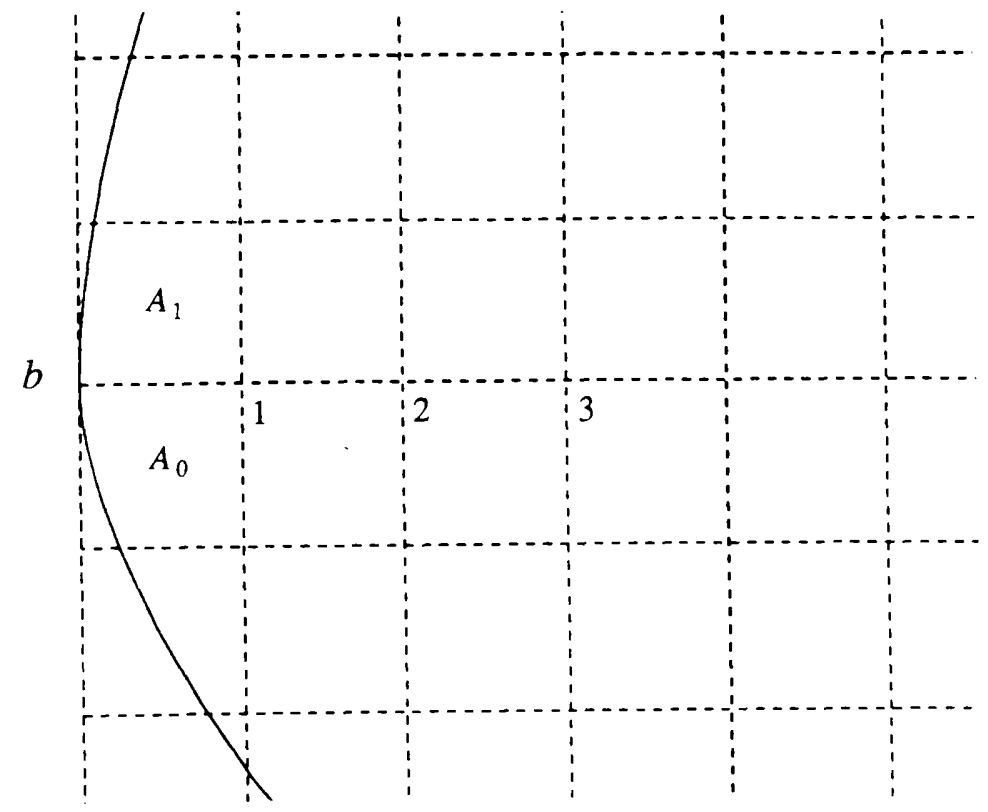

Figure 3.4

The unknown boundary value of $B_{2}$ at $b$ is updated in such a way that ensures flux conservation. 


\section{Chapter 4}

\section{Toroidal Effects}

The most promising type of plasma containment devices have toroidal geometries. It is therefore essential to consider solutions in toroids and to see what difference the curvature makes on solutions obtained in the rectangular case.

\subsection{D Solutions}

Consider the solid to be in the form of a toroid whose cross-section is rectangular. The solid is surrounded by a perfectly conducting shell and is driven by a constant toroidal electric field applied at the rim.

Solutions using the stream function and components will be sought when there is no $\phi$ dependence.

\subsubsection{Stream function method}

Referring to figure 4.1 the toroidal co-ordinate system, ( $\mathrm{x}, \mathrm{y}, \phi)$ can be defined, using the Cartesians ( $\mathrm{X}, \mathrm{Y}, \mathrm{Z}$ ), by the following transformations :

$$
X=x
$$




$$
Y=\left(R_{o}+y\right) \cos \phi=R \cos \phi
$$

$$
Z=\left(R_{o}+y\right) \sin \phi=R \sin \phi
$$

A simple calculation yields the following scale factors

$$
h_{x}=h_{y}=1 \quad \text { and } \quad h_{0}=R
$$

The divergence-free constraint can be written as

$$
\nabla \cdot \mathbf{B}=\frac{1}{R}\left\{\frac{\partial}{\partial x}\left(R B_{x}\right)+\frac{\partial}{\partial y}\left(R B_{y}\right)\right\}=0
$$

This suggests defining the stream function as :

$$
\begin{aligned}
& R B_{x}=\frac{\partial \psi}{\partial y} \\
& R B_{y}=-\frac{\partial \psi}{\partial x}
\end{aligned}
$$

In terms of $\psi$ and $B_{\phi}$ the evolution equations reduce to :

$$
\frac{\partial \psi}{\partial t}=\nabla^{2} \psi-\frac{1}{R} \frac{\partial \psi}{\partial x}-(1-\varepsilon) R F B_{\phi}+\alpha R
$$


$\frac{\partial B_{\diamond}}{\partial t}=\nabla^{2} B_{\diamond}+\frac{\partial}{\partial x}\left(\frac{B_{\emptyset}}{R}\right)+(1-\varepsilon)\left\{\frac{\partial}{\partial x}\left[\frac{F}{R} \frac{\partial \psi}{\partial x}\right)+\frac{\partial}{\partial y}\left(\frac{F}{R} \frac{\partial \psi}{\partial y}\right)\right\}$

where

$$
F=\frac{R\left\{B_{\diamond} \nabla^{2} \psi-\frac{\partial \psi}{\partial x} \frac{\partial B_{\circ}}{\partial x}-\frac{\partial \psi}{\partial y} \frac{\partial B_{\circ}}{\partial y}\right\}-2 B_{\phi} \frac{\partial \psi}{\partial x}}{(\nabla \psi)^{2}+\left(R B_{\phi}\right)^{2}}
$$

The $B_{\phi}$ evolution equation has been written in a conservative type form in order to ensure flux conservation.

\subsubsection{Boundary conditions and their implementation}

The boundary conditions for toroidal boundaries are altered due to the curvature as are the evolution equations. It has been shown that they can be written in terms of $\psi$ as :

$$
\begin{aligned}
& \frac{\partial}{\partial x}\left(R B_{\phi}\right)=\alpha R^{2}(1-\varepsilon) \frac{\psi_{; x} B_{\phi}}{\psi_{; x}^{2}+\left(R B_{\phi}\right)^{2}} \\
& \frac{\partial}{\partial x}\left[\frac{1}{R} \frac{\partial \psi}{\partial x}\right)=-\alpha \frac{\left(\varepsilon \psi_{; x}^{2}+\left(R B_{\phi}\right)^{2}\right)}{\psi_{; x}^{2}+\left(R B_{\phi}\right)^{2}}
\end{aligned}
$$

on ad, bc and : 


$$
\frac{\partial B_{\circ}}{\partial y}=\alpha R(1-\varepsilon) \frac{\psi_{; y} B_{\phi}}{\psi_{; y}^{2}+\left(R B_{\phi}\right)^{2}}
$$

$$
\frac{\partial^{2} \psi}{\partial y^{2}}=-\alpha R \frac{\left(\varepsilon \psi_{; y}^{2}+\left(R B_{0}\right)^{2}\right)}{\psi_{; y}^{2}+\left(R B_{\phi}\right)^{2}}
$$

on $\mathrm{ab}$ and $\mathrm{cd}$.

These can be implemented as previously by using central and forward or backward difference representations. Flux conservation is assured as in previous methods.

\subsubsection{Solution Using Components}

The evolution equations in terms of the components of $\mathbf{B}$ take on the form :

$$
\frac{\partial B_{x}}{\partial t}=\nabla^{*} B_{x}-\frac{B_{x}}{R^{2}}-(1-\varepsilon)\left\{B_{\phi} \frac{\partial F}{\partial y}+F \frac{\partial B_{\phi}}{\partial y}\right\}
$$

$$
\frac{\partial B_{y}}{\partial t}=\nabla^{*} B_{y}+\frac{(1-\varepsilon)}{R}\left\{F B_{\phi}+F B_{\phi} \frac{\partial F}{\partial x}+R F \frac{\partial B_{\circ}}{\partial x}\right\}
$$

$$
\frac{\partial B_{\circ}}{\partial t}=\nabla^{*} B_{\phi}+\frac{\partial}{\partial x}\left[\frac{B_{\phi}}{R}\right)(1-\varepsilon)\left\{\frac{\partial}{\partial x}\left(F B_{y}\right)-\frac{\partial}{\partial y}\left(F B_{x}\right)\right\}
$$

where $\nabla^{*} \equiv \frac{\partial^{2}}{\partial x^{2}}+\frac{\partial^{2}}{\partial y^{2}}+\frac{1}{R} \frac{\partial}{\partial x}$ 
To satisfy the divergence-free condition automatically, they can be written as :

$$
\frac{\partial B_{x}}{\partial t}=\frac{\partial}{\partial y}\left[\frac{\partial B_{x}}{\partial y}\right]-\frac{\partial^{2} B_{y}}{\partial y \partial x}-(1-\varepsilon) \frac{\partial}{\partial y}\left(F B_{0}\right)
$$

$$
\frac{\partial B_{y}}{\partial t}=\frac{1}{R} \frac{\partial}{\partial x}\left[R \frac{\partial B_{y}}{\partial x}\right]-\frac{1}{R} \frac{\partial}{\partial x}\left[R \frac{\partial B_{x}}{\partial y}\right]+\frac{(1-\varepsilon)}{R} \frac{\partial}{\partial x}\left(R F B_{\diamond}\right)
$$

$$
\frac{\partial B_{\diamond}}{\partial t}=\frac{\partial^{2} B_{\diamond}}{\partial x^{2}}+\frac{\partial^{2} B_{\diamond}}{\partial y^{2}}+\frac{\partial}{\partial x}\left[\frac{B_{\diamond}}{R}\right]-(1-\varepsilon)\left\{\frac{\partial}{\partial x}\left(F B_{y}\right)-\frac{\partial}{\partial y}\left(F B_{x}\right)\right\}
$$

\subsubsection{Boundary conditions}

In terms of components the boundary conditions take on the form :

$$
\begin{aligned}
& \frac{\partial B_{y}}{\partial x}=\alpha \frac{\left(\varepsilon B_{y}^{2}+B_{o}^{2}\right)}{\left(B_{y}^{2}+B_{\phi}^{2}\right)} \\
& \frac{\partial}{\partial x}\left(R B_{\phi}\right)=\alpha R(\varepsilon-1) \frac{B_{y} B_{\phi}}{\left(B_{y}{ }^{2}+B_{\phi}^{2}\right)} \\
& B_{x}=0
\end{aligned}
$$


on ad, bc and :

$$
\begin{aligned}
& \frac{\partial B_{x}}{\partial y}=-\alpha \frac{\left(\varepsilon B_{x}^{2}+B_{\phi}^{2}\right)}{\left(B_{x}^{2}+B_{\phi}^{2}\right)} \\
& \frac{\partial B_{\phi}}{\partial y}=\alpha(1-\varepsilon) \frac{B_{x} B_{\phi}}{\left(B_{x}^{2}+B_{\phi}^{2}\right)} \\
& B_{y}=0
\end{aligned}
$$

on $\mathrm{ab}$ and $\mathrm{cd}$.

These can be implemented using central difference formulae and axial flux conservation is achieved as previously.

The evolution equations have been written such that $\nabla \cdot \mathbf{B}=0$ is satisfied automatically. As stated previously this will be true provided points adjacent to the point being considered are calculated using the evolution equations. This is not the case when calculating updated values at interior boundary neighbours as the boundary condition $\mathbf{n} \cdot \mathbf{B}=0$ is used to assign values at the boundary. It has been shown that such a problem does not arise when considering Cartesian co-ordinates but in toroidal co-ordinates a non vanishing term arises (Appendix D \}. The problem can be overcome by using the divergence-free constraint itself as in section 2.1.6. 


\subsection{D Solution}

Consider a toroidal container whose cross-section is rectangular and is surrounded by a perfectly conducting shell. The system is driven by a constant toroidal electric field applied throughout the rim as a first case and across poloidal gaps in the second. The evolution equations can be written as :

$$
\begin{aligned}
\frac{\partial B_{x}}{\partial t} & =\nabla^{*} B_{x}-\frac{B_{x}}{R^{2}}-\frac{2}{R^{2}} \frac{\partial B_{\phi}}{\partial \phi} \\
& -(1-\varepsilon)\left\{B_{\phi} \frac{\partial F}{\partial y}+F \frac{\partial B_{\phi}}{\partial y}-\frac{B_{y}}{R} \frac{\partial F}{\partial \phi}-\frac{F}{R} \frac{\partial B_{y}}{\partial \phi}\right\}
\end{aligned}
$$

$$
\begin{aligned}
\frac{\partial B_{y}}{\partial t}=\nabla^{*} B_{y} \\
+\frac{(1-\varepsilon)}{R}\left\{F B_{\phi}+F B_{\phi} \frac{\partial F}{\partial x}+R F \frac{\partial B_{\phi}}{\partial x}-B_{x} \frac{\partial F}{\partial \phi}-F \frac{\partial B_{x}}{\partial \phi}\right\} \\
\frac{\partial B_{\phi}}{\partial t}=\frac{\partial^{2} B_{\phi}}{\partial x^{2}}+\frac{\partial^{2} B_{\phi}}{\partial y^{2}}+\frac{\partial}{\partial x}\left(\frac{B_{\phi}}{R}\right)-\frac{\partial}{\partial x}\left[\frac{1}{R} \frac{\partial B_{x}}{\partial \phi}\right)-\frac{1}{R} \frac{\partial^{2} B_{y}}{\partial y \partial \phi} \\
-(1-\varepsilon)\left\{\frac{\partial}{\partial x}\left(F B_{y}\right)-\frac{\partial}{\partial y}\left(F B_{x}\right)\right\}
\end{aligned}
$$


where $\nabla^{*} \equiv \frac{\partial^{2}}{\partial x^{2}}+\frac{\partial^{2}}{\partial y^{2}}+\frac{1}{R^{2}} \frac{\partial^{2}}{\partial \phi^{2}}+\frac{1}{R} \frac{\partial}{\partial x}$

Alternatively, to satisfy $\nabla \cdot \mathbf{B}=0$ automatically, they can be written as :

$$
\begin{aligned}
\frac{\partial B_{x}}{\partial t} & =\frac{\partial}{\partial y}\left[\frac{\partial B_{x}}{\partial y}\right]-\frac{\partial^{2} B_{y}}{\partial y \partial x}+\frac{1}{R^{2}} \frac{\partial}{\partial \phi}\left(\frac{\partial B_{x}}{\partial \phi}\right]-\frac{1}{R^{2}} \frac{\partial^{2}\left(R B_{0}\right)}{\partial \phi \partial x} \\
& -(1-\varepsilon)\left\{\frac{\partial}{\partial y}\left(F B_{\phi}\right)-\frac{\partial}{\partial \phi}\left(F B_{y}\right)\right\} \\
\frac{\partial B_{y}}{\partial t} & =\frac{1}{R^{2}} \frac{\partial}{\partial \phi}\left[\frac{\partial B_{y}}{\partial \phi}\right]-\frac{1}{R} \frac{\partial^{2} B_{\phi}}{\partial \phi \partial y}+\frac{1}{R} \frac{\partial}{\partial x}\left[R \frac{\partial B_{y}}{\partial x}\right]-\frac{1}{R} \frac{\partial^{2}\left(R B_{x}\right)}{\partial x \partial y} \\
& -\frac{(1-\varepsilon)}{R}\left\{\frac{\partial}{\partial y}\left(F B_{x}\right)-\frac{\partial}{\partial \phi}\left(R F B_{\phi}\right)\right\}
\end{aligned}
$$

$$
\begin{aligned}
\frac{\partial B_{\phi}}{\partial t} & =\frac{\partial}{\partial x}\left[\frac{1}{R} \frac{\partial}{\partial x}\left(R B_{\phi}\right)\right]+\frac{\partial}{\partial y}\left[\frac{\partial B_{\phi}}{\partial y}\right]-\frac{\partial}{\partial x}\left[\frac{1}{R} \frac{\partial B_{x}}{\partial \phi}\right]-\frac{1}{R} \frac{\partial^{2} B_{y}}{\partial y \partial \phi} \\
& -(1-\varepsilon)\left\{\frac{\partial}{\partial x}\left(F B_{y}\right)-\frac{\partial}{\partial y}\left(F B_{x}\right)\right\}
\end{aligned}
$$

The problem of axial flux conservation can be overcome as in previous sections. 
The boundary conditions are no different to the $2 \mathrm{D}$ case and are implemented as before.

At interior boundary neighbours the magnetic field is not divergence-free and the problem can be overcome as in the previous section.

In the next chapter we present some of the interesting results obtained using the suite of codes written. 


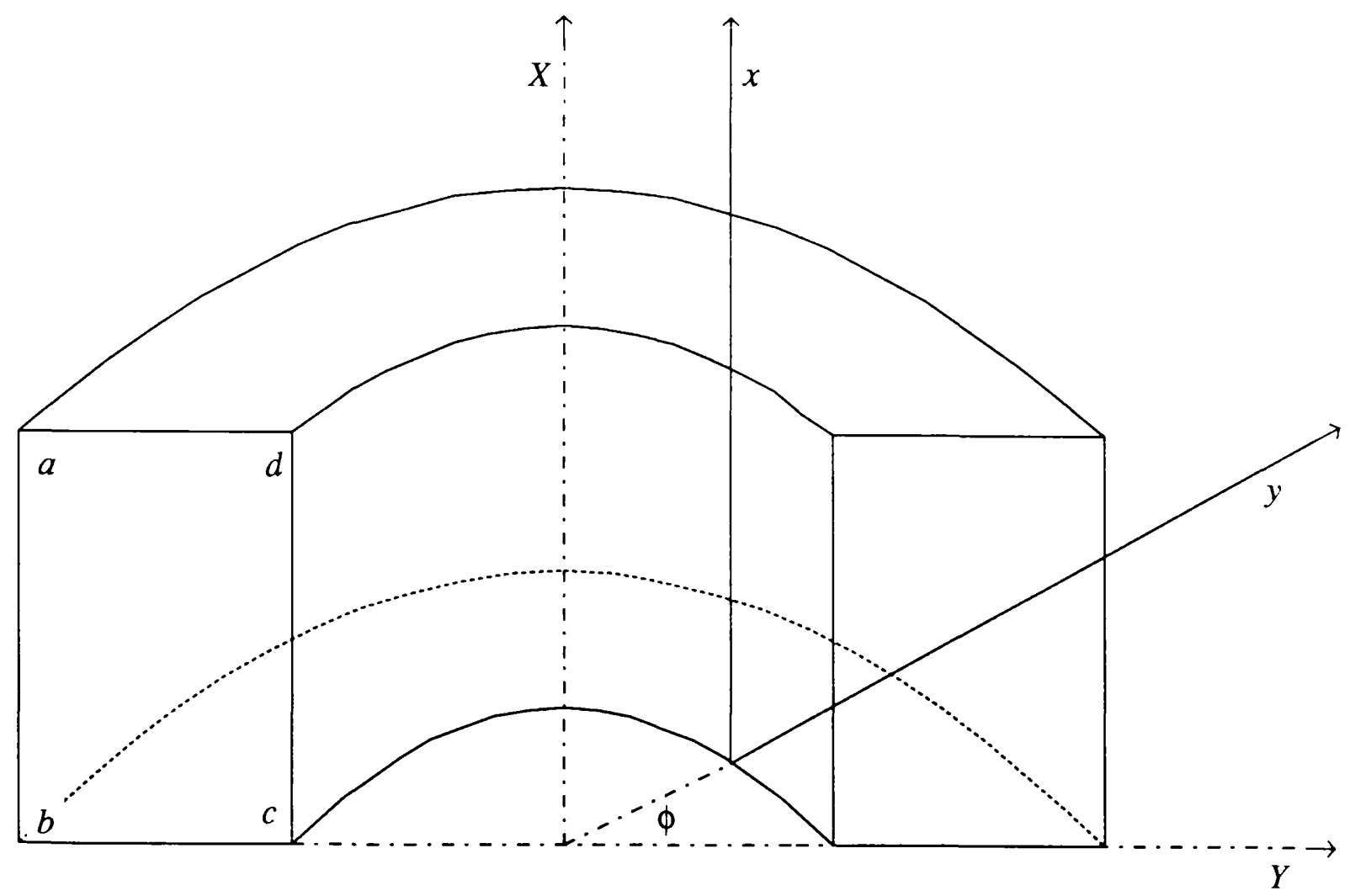

Figure 4.1

Co-ordinate system used for toroidal calculations. 


\section{Chapter 5}

\section{Results and Conclusions}

In this chapter we compare results produced by the present theory with experimental data, Taylor's theory and simulations carried out by others.

As was mentioned in in chapter (2) a suitable $\varepsilon$ must be chosen. This is the smallest possible $\varepsilon$ and is therefore determined by the accuracy of the numerical scheme. Figure 5.1 shows the time development of $|\mathbf{j} \times \mathbf{B}|_{\max }$ for diminishing values of epsilon. In each run the same initial configuration is chosen which is far from force-free i.e. $|\mathbf{j} \times \mathbf{B}|_{\max }(t=0)=513$.3. The relaxation time i.e. the time taken to reach a stationary state has been normalised to unity and the results are presented just after $|\mathbf{j} \times \mathbf{B}|_{\max }$ falls below unity. The results correspond to a square crosssection represented by $10 \times 10$ mesh points. The two time scales associated with the parallel and perpendicular conductivities are clearly visible. In each case there is a rapid equilibration to a force-free field, $|\mathbf{j} \times \mathbf{B}| \rightarrow O(\varepsilon)$, after which there is a much slower evolution through a sequence of force-free equilibria. In each case the final state is force-free to $O(\varepsilon)$.

Before presenting any results, a few important points should be highlighted about the value of $\mathbf{j} \times \mathbf{B}$ at a boundary. This is important as we have considered boundary conditions which do not allow tangential electric fields and yet we have an externally applied tangential driving field. Having a tangential electric field is inconsistent with the force-free constraint and we must therefore understand the effect the inconsistency has on the constraint. Consider a rectangular cross-section and a boundary where $B_{x}=0$ as in chapter (2). On this boundary : 


$$
\frac{\partial B_{z}}{\partial x}=\frac{\alpha(\varepsilon-1) B_{y} B_{z}}{B_{y}{ }^{2}+B_{z}{ }^{2}} \quad, \quad \frac{\partial B_{y}}{\partial x}=\frac{\alpha\left(\varepsilon B_{y}{ }^{2}+B_{z}{ }^{2}\right)}{B_{y}{ }^{2}+B_{z}{ }^{2}}
$$

and

$$
\mathbf{j} \times \mathbf{B}=\left(-B_{z} \frac{\partial B_{z}}{\partial x}-B_{y} \frac{\partial B_{y}}{\partial x},-B_{z} \frac{\partial B_{z}}{\partial y}, B_{y} \frac{\partial B_{z}}{\partial x}\right)
$$

Substitution then gives :

$$
\mathbf{j} \times \mathbf{B}=\left(-\alpha \varepsilon B_{y} B_{z},-B_{z} \frac{\partial B_{z}}{\partial y}, B_{y} \frac{\partial B_{z}}{\partial y}\right)
$$

Because $B_{z}=B_{z}(\psi)$ and $\psi$ is constant on the boundary, all the above terms are $O(\varepsilon)$. The force-free constraint is thus satisfied, to the required order, at an interface. This is assuming that currents are allowed to flow at the boundary. If $\mathbf{n} \times \mathbf{j}=0$, then :

$$
\mathbf{j} \times \mathbf{B}=\left(-\alpha B_{y} \quad,-B_{z} \frac{\partial B_{z}}{\partial y}, B_{y} \frac{\partial B_{z}}{\partial y}\right)
$$

From the first component it is clear that the driving term must be chosen sufficiently small in order to ensure that the force-free constraint remains satisfied, to the required order, at an interface.

The inconsistency introduced by the driving field is therefore seen to have little effect on the force-free nature of the evolution. This is essentially because the 
force-free condition needs to be zero only to order $\varepsilon$ and having a suitably chosen driving field does not violate this condition. For high values of driving field $|\mathbf{j} \times \mathbf{B}|_{\max }$, at the boundary, is found to drift from $O(\varepsilon)$ as expected.

The inconsistency has important consequences when considering force-free solutions to the MHD equations directly. Since the solutions must be exactly force-free, rather than to a certain order, no solutions can be obtained. The problem can only be overcome by relaxing the force-free constraint or by using a different set of boundary conditions.

We next present some results obtained for the $2 \mathrm{D}$ case.

\section{D-Solutions}

Consider a square cross-section represented by $10 \times 10$ mesh points. The value of $\varepsilon$ is chosen to be $10^{-2}$, unless stated, for every result. The results are for the case when there are no surface currents.

As stated in (1.5), experimental data is sometimes represented by $F-\theta$ diagrams, where :

$$
F=\frac{B_{z o}}{\left\langle B_{z}\right\rangle} \quad \text { and } \quad \theta=\frac{I_{z}}{\left\langle B_{z}\right\rangle Q}
$$

$I_{z}$ is the total axial current, $B_{z 0}$ is the value of the axial field at the boundary, $\left\langle B_{z}\right\rangle$ is the average axial field across the cross-section and $a$ is just the total length of the boundary. The $\theta$ parameter is defined such that it reduces to the one which is usually considered for circular cross-sections involving the value of the 
poloidal field at the boundary. It should be noted that $F<0$ indicates a field reversal at the boundary.

Since there are no experimental devices possessing square cross-sections a quantitative comparison with experiment cannot be made. But results can be compared with Taylor's theory and qualitative features of experimental data. Figure 5.2 shows the locus of relaxed states obtained by varying the axial flux and by considering three different values of driving field ( $\alpha=0.1,0.3$ and 0.5 ) as indicated by the three different symbols. The result predicted by Taylor's theory [5.1] is also shown. As can be seen all relaxed states lie on a single universal curve which is in agreement with Taylor's theory. This universal nature is also found experimentally ( figure 1.1 ) [1.11] albeit for circular cross-section devices. Another important result predicted by the experimental $F-\theta$ diagram is the point at which the magnetic field direction at the boundary reverses. Field reversal is predicted, by the present theory, to take place at $\theta \approx 2.0$ which can be compared with that predicted by Taylor's [5.1] theory of $\theta=1.2$. The results can also be compared with a recent simulation performed by Kirby [5.2] shown in figure 5.2b. This result agrees well with the present theory though the results are for circular cross-sections. The discrepancy with experimental data has been attributed [5.2] to the unrealistic approximation of constant resistivity. Choosing a structured resistivity, consistent with experimental data, gives a much more accurate profile as is also shown in figure $5.2 \mathrm{~b}$. The present theory is, of course, only, valid for a constant resistivity and so results predicted by a structured resistivity cannot be generated.

The relaxed profiles corresponding to $F<0$ are those arising in Reversed Field Pinches ( RFP ) [1.2] and the others arise in Tokamaks [1.2]. Since RFP states are obtained, the generation of field reversal can thus be attributed purely to a nonlinear diffusive process. 
In Taylor's theory the solution to $\nabla \times \mathbf{B}=\mu \mathbf{B}$ gives the relaxed profile for minimum $\mu$ which corresponds to minimum energy. There is in fact a simple relation between $\mu$ and $\theta$. For rectangular cross-sections, of dimension $a b$, it is easy to show that

$$
\theta=\frac{\mu}{2} \frac{a b}{a+b}
$$

The pinch parameter is thus proportional to $\mu$. This then implies a universal relation for a $\theta$ versus $\mu$ and thus $F$ versus $\mu$ plot. In the present theory $\mu$ is not a constant, over the cross-section $S$, and can be defined as an average $\langle\mu\rangle$, where

$$
<\mu>=\frac{1}{a b} \int_{S} \frac{\nabla \times \mathbf{B} \cdot \mathbf{B}}{B^{2}} d S
$$

Typical profiles of $\mu$ are shown in figure $5.2 \mathrm{c}$ and should be compared to those obtained by Kirby [5.2] and experiment as shown in figure $5.2 \mathrm{~d}$.

Figures 5.3 and 5.4 show a plot of $F$ versus $\langle\mu>$ and $\theta$ versus $\langle\mu>$ together with the results predicted by Taylor's theory. Once again universal profiles are generated. It should be noted that $\theta$ varies linearly with $\langle\mu\rangle$ only for small $\theta$. It can also be seen that there is an upper limit on $\langle\mu\rangle$. This is also the case in Taylor's theory [5.1] where $\mu_{\max }=6.8$ and $\theta_{\max }=1.7$. The present theory does not predict an upper limit on $\theta$.

Another pair of universal curves can be obtained by plotting $\left\langle j_{z}>/ \alpha\right.$ versus $\theta$ or $\mu$ as in figures 5.5 and 5.6. They show that the toroidal current, at constant $\theta$ or $\langle\mu\rangle$, is proportional to the externally applied driving field. They also predict a limit on the toroidal current for a fixed value of $\alpha$. 
It can be seen from most plots that there appears to be a region along which no data points have been obtained. Why this is so can be seen by plotting $\left\langle j_{z}>/ \alpha\right.$ versus $\Phi / \alpha$, ( $\Phi=$ axial flux ), as in Figure 5.7. For a fixed driving field, the toroidal current initially rises for an increase in axial flux, reaches a maximum and then begins to decrease. The toroidal flux then reaches a critical value at which there is a sudden drop in toroidal current. This then reveals equilibrium states which are inaccessible by the plasma. In other words there exists a value of toroidal flux, for a fixed $\alpha$, at which the final states are unstable and which may result in a sudden drop or sudden rise in the toroidal current. The plot also shows the existence of states which have a different axial flux but the same amount of toroidal current. It can also be concluded that about the critical value of flux the are two modes at which the system can operate. The first corresponding to the low current and the second to the high one. This lower and upper stable current operation is also found in Tokamak experiments [5.3] where a disruptive instability causes a rapid drop in the toroidal current. Figure $5.7 \mathrm{~b}$ shows the time development of the axial current, $F$ and $\theta$ for a case where the current actually rises. Initially, the driving field gives rise to an axial current which increases until it reaches a constant value. It remains constant and then rapidly rises. It should be noted that we have been considering the toroidal current rather than the total current. This is because it is the toroidal component that yields universal profiles. Although it appears that it is only the toroidal current which may rapidly rise or fall it is in fact true that the total current behaves in the same way. In figure $5.7 \mathrm{c}$ we have shown how the total current varies with the axial flux. The plot is virtually identical to figure 5.7 except that here there is a contribution from the poloidal current. The poloidal current is, of course, not large enough to cause a vertical shift which could remove the 'sudden drop' region.

An important parameter in instability theory is the $q$ value. We initially considered an average defined by 


$$
<q>=\int_{s} \frac{\left(B_{x}^{2}+B_{y}{ }^{2}\right)}{B_{z}} d s
$$

We have placed the axial component as the denominator because both $B_{x}$ and $B_{y}$ being zero at the corners would cause singularity problems. A plot of $<q>$ versus $\Phi / \alpha$ is found to give a universal curve as is shown in figure $5.7 \mathrm{~d}$. We next defined it the traditional way as

$$
\frac{1}{\langle q\rangle}=\int_{s} \frac{\left(B_{x}{ }^{2}+B_{y}{ }^{2}\right)}{r B_{z}} d s
$$

where $r^{2}=x^{2}+y^{2}$. This definition also gives a universal curve as is shown in figure $5.7 \mathrm{e}$.

The rapid rise or fall of the toroidal current is feature of toroidal discharges that is not predicted by Taylor's theory. Here $j_{z}=\mu B_{z}$ and so $I_{z}=\mu \Phi$. The axial current, at fixed axial flux, is thus proportional to $\mu$ which is assumed to be continuous. In the present theory $\mu$ is not a constant. The axial current is then, after integrating by parts,

$$
\begin{aligned}
I_{z} & =\left[\mu \Delta \Phi-\int\left[\Delta \Phi \frac{d \mu}{d s}\right) d s\right]_{s=0}^{s=A} \\
& =\left\langle\mu>\Phi-\left[\int\left[\Delta \Phi \frac{d \mu}{d s}\right)\right]_{s=0}^{s=A}\right.
\end{aligned}
$$

This result, for non-constant $\mu$, is clearly different from Taylor's case. 
Another pair of interesting curves are obtained by plotting the plasma magnetic energy $E$ versus $\Phi$ and $<j_{2}>$ versus $E$ as in figures 5.9 and 5.10. From figure 5.9 it can be concluded that the energy is not a continuous function of axial flux. About the critical value of axial flux there exist different states having the same energy i.e. there exist degenerate states.

It can also be seen from the universal curves that if a value of one plotted parameter is given then the equilibrium state is completely defined. From Figure 5.7 it can be concluded that if the toroidal flux and the driving field are given then the final state is uniquely determined. These two quantities are prescribed as an initial condition and so the final state depends only on the initial values of $\Phi$ and $\alpha$. In Taylor's theory the final state of a plasma is uniquely determined once the toroidal flux and the global helicity are given. But the helicity, at constant toroidal flux, is proportional to the volt-seconds stored in the discharge [5.4]. So once the voltseconds, which is the driving term, and the toroidal flux are given the final state can be determined. The present theory agrees well with this prediction.

In figures 5.10 and 5.11 we show the time development of $F$ and $\theta$ for which the relaxed state corresponds to a tokamak and RFP profile. The corresponding equilibrium profiles are shown in figures 5.12 and 5.13. These should be compared with those shown in figure 1.1. The time development of $F$ and $\theta$ as obtained by Kirby [5.2] are shown in figure $5.11 \mathrm{~b}$.

The results presented so far are those for a square cross-section. For a $1 \times 2$ rectangle, similar results are obtained. Figure 5.14 shows the $F-\theta$ plot together with the result predicted by Taylor's theory. As can be seen, the profile is very similar to the square case and there is only a slight difference in the field reversal values. This insignificant dependence agrees well with Taylor's theory. 
When considering the model where surface currents are allowed to flow, there is a considerable difference in the results. Figure 5.15 shows the $F-\theta$ profile for a square cross-section. Once again, a universal curve is produced and for small $\theta$ the profile is very similar to that predicted by Taylor's theory. But the important difference is that no reversed field configurations are generated. This model is very similar to the force-free paramagnetic model ( FFPM ) $[5.5,1.11]$, the results of which are shown in figure 5.16 .

In the first model the derivative boundary conditions were obtained by demanding that $\mathbf{n} \times \mathbf{E}$ vanish and Ohm's Law was used to achieve this. No constraint was placed on the currents at the boundary. Consider Ohm's Law :

$$
\mathbf{E}=a F \mathbf{B}+b \mathbf{j}
$$

then, for force-free fields

$$
\mathbf{j} \times \mathbf{E}=a F \mathbf{j} \times \mathbf{B} \approx 0 \quad \Rightarrow \quad \mathbf{E} \propto \mathbf{j}
$$

It then follows that if $\mathbf{n} \times \mathbf{E}$ vanishes at an interface then so does $\mathbf{n} \times \mathbf{j}$. We would then expect equilibrium profiles to be similar to the case where we impose $\mathbf{n} \times \mathbf{j}=0$ directly rather than $\mathbf{n} \times \mathbf{E}=0$. This is clearly not the case. The reason why this is not so can be seen by noting that $\mathbf{j} \times \mathbf{B}$ is zero only to order $\varepsilon$. But this term contains the contribution from the driving field. Therefore, a change in the driving field may only cause variations in $\mathbf{n} \times \mathbf{E}$ and $\mathbf{n} \times \mathbf{j}$ of order $\varepsilon$ but the global solution changes considerably.

In the current-free case a constant $j_{z}$ was used as the driver and $E_{y}$ and $E_{z}$ were able to exist ( order $\varepsilon$ ). In the other case a constant $E_{z}$ was the driver and $j_{y}$ and $j_{z}$ were able to exist ( again order $\varepsilon$ ). It is also possible to have $j_{y}$ vanish and have 
a constant $E_{z}$ as the driver. The boundary conditions, where $B_{x}$ vanishes, then turn out to be :

$$
\frac{\partial B_{y}}{\partial x}=\frac{\alpha\left(B_{y}{ }^{2}+B_{z}{ }^{2}\right)}{B_{y}{ }^{2}+\varepsilon B_{z}{ }^{2}} \quad \text { and } \quad \frac{\partial B_{z}}{\partial x}=0 \quad \text { where } \quad \alpha=\sigma_{\perp} C
$$

This model is found to give unacceptable results. No universal profiles are generated for instance. Results for this model will thus not be presented. It does, however, highlight the fact that the boundary conditions imposed on $\mathbf{B}$, arising from Ohm's Law, play a critical role in defining equilibrium profiles. This was also realised after considering the current and current-free surface models. If this is so, then it has important implications for the well known method [5.6] of obtaining axisymmetric equilibria which involves finding solutions to the Grad-Shafranov equation. There, the boundary condition $\mathbf{n} \cdot \mathbf{B}=0$ implies that $\psi$ be constant on the boundary and no account of Ohm's law is taken. Ohm's Law can be written as

$$
\mathbf{E}+\mathbf{V} \times \mathbf{B}=\eta \mathbf{j}
$$

In ideal $\mathrm{MHD}, \mathbf{E}$ at a perfectly conducting boundary is not coupled to $\mathbf{B}$, provided $\mathbf{n} \cdot \mathbf{V}=0$. Now, for finite resistivity problems, we must ensure that $\mathbf{n} \times \mathbf{E}=0$. This means that, if we have $\mathbf{n} \cdot \mathbf{V}=0$, we must ensure that $\mathbf{n} \times \mathbf{j}=0$. This constrains the normal derivatives of the tangential components $B_{z}$ and $B_{y}$, where $B_{x}=0$, which means that there is an additional constraint on $\psi$ at the boundary. It should also be noted that the Grad-Shafranov equation is solved after a current profile is prescribed. This assumes that such a profile is dynamically accessible. We have shown that some states are not possible to relax to and so care must be taken when prescribing a current profile. 
Another interesting feature of relaxed states predicted by Taylor's theory is that when toroidal effects are taken into account the curvature has very little effect on the $F-\theta$ profile [5.7].

The $F-\theta$ parameters can be defined in toroidal co-ordinates as :

$$
F=\frac{\left(R B_{\emptyset}\right)_{\mathrm{o}}}{\left\langle R B_{\phi}\right\rangle} \quad \text { and } \quad \theta=\frac{\int R j_{\phi} d s}{\left\langle R B_{\phi}\right\rangle C}
$$

The coefficient $R$ has appeared in these definitions due to $R B_{\phi}$ having a functional dependence on $\psi$ rather than just the toroidal component as in the cylindrical case. In the limit of large-aspect-ratio, these definitions reduce to the cylindrical case as is required.

The predicted field reversal values for a unit and infinite aspect ratio device are 1.24 and 1.11 respectively. The insignificant dependence on the curvature is also found experimentally where many devices of differing aspect ratio yield very similar $F-\theta$ profiles. The results predicted by the present theory are presented in figure 5.17. Once again there is very good agreement with Taylor's theory.

It should be noted that it is the $F-\theta$ profile which varies insignificantly. The profiles of $j_{z}, \mathbf{B}$ etc are of course significantly different. Figure 5.18 shows three $<j_{z}>$ versus $\Phi$ profiles for the aspect ratios being considered. It should be noted that the critical flux value, at which there may be a rapid fall or rise in axial current, is lowest for the smallest aspect ratio but the critical value is higher for aspect ratio 4.0 than 10.0 . The critical flux value thus increases with aspect ratio, reaches a maximum and then begins to fall. There is therefore a critical aspect ratio at which the critical flux value is maximum. Figure $5.18 \mathrm{~b}$ shows a plot of critical flux $\Phi_{C}$ versus aspect-ratio. The critical aspect-ratio can seen to be $\approx 3.0$. This is 
the most desirable value to build a device since at this aspect-ratio, the device can be operated at highest toroidal current before reaching the critical flux value.

Upto now we have concentrated on understanding equilibrium profiles and have mentioned very little about the global evolution of the plasma. The nature of the evolution will for obvious reasons depend on the initial configuration. We consider an initial state which consist of several island formations. Consider a $1 \times 2$ rectangular cross-section represented by $10 \times 20$ mesh points. The initial configuration, figure 5.19 , consists of four islands which represent four helices all of which have the same handedness. As can be seen, the helices whose axis are the closest reconnect first to give just two islands. These in turn reconnect to give a simple single island as the final relaxed state. In ideal MHD the four initial islands would of course retain their structure and reconnection would not take place.

As a second run, consider a $1 \times 3$ rectangle and single island structure as an initial condition ( figure $5.19 \mathrm{~b}$ ). The axial electric field inside the plasma is opposite to that of the driving field. Reconnection gives rise to multiple island formation which subsequently form a single island as a final equilibrium state.

\section{D-Solutions}

In $3 \mathrm{D}$ it is informative to view the structure of magnetic field lines. The equations of the field lines can be obtained using $\mathbf{d r} \times \mathbf{B}=0$.

$$
\mathbf{d r} \times \mathbf{B}=0 \quad \Rightarrow \quad \frac{d x}{B_{x}}=\frac{d y}{B_{y}}=\frac{d z}{B_{z}}
$$


The line element is given by $d l^{2}=d x^{2}+d y^{2}+d z^{2}$.

From these two it follows that :

$$
\frac{d x}{d l}=\frac{B_{x}}{B} \quad, \quad \frac{d y}{d l}=\frac{B_{y}}{B} \quad \text { and } \quad \frac{d z}{d l}=\frac{B_{z}}{B}
$$

These equations can be integrated, to give the co-ordinates $(x, y, z)$ of $\mathbf{B}$, using the fourth order predictor-corrector Adams-Moulton method [5.8]. This requires three known values to get started which can be obtained using the Runge-Kutta method.

As a test run consider a $1 \times 1 \times 2$ rectangular cylinder represented by $10 \times 10 \times 20$ mesh points. An initial configuration is chosen to be far from the relaxed state ( figure 5.20 (i), $\mathrm{T}=0$ ). $\mathrm{T}$ is proportional to the number of time steps. Here, the handedness of the initial he lical structure is chosen to be opposite to that of the relaxed state. The initial state is also given an axial dependence as the driving field is applied throughout the rim.

Figures 5.20 (i), 5.20 (ii) and 5.20 (iii) show the time development of the magnetic field lines. During the first few time steps the initial configuration rapidly looses its axial dependence. The system then becomes very active towards the boundaries ( $\mathrm{T}=100$ ). The handedness of the helices is still the same as that of the initial state. The activity seems to die away after this stage $(T=110)$ but the system soon becomes very active again ( $T=170$ ). At $T=200$ the helices have begun to change handedness. This seems to have first originated at the ends of the cylinder. The helices continue to change handedness until reaching the central region of the cylinder ( $\mathrm{T}=240$ ). Here reconnection has given rise to field reversal. The field reversal begins to disappear as the configuration approaches an axisymmetric state ( 
$\mathrm{T}=245$ ). As can be seen, the final relaxed state is a very simple axisymmetric profile ( $\mathrm{T}=500$ ). The final state is in fact an RFP configuration and the field reversal has not been shown. The code is therefore able to handle highly active phenomena and possible reconnection.

In Taylor's theory, solutions to $\nabla \times \mathbf{B}=\mu \mathbf{B}$ gives the relaxed profile. Of all the eigenvalues allowed, the minimum is to be chosen as this corresponds to minimum energy. The general solutions to this equation have been obtained by Chandrasekhar and Kendal [5.9]. For a large-aspect-ratio toroidal device, they can be written [5.4] as :

$$
\begin{gathered}
B_{r}^{m k}=\frac{-1}{\left(\mu^{2}-k^{2}\right)^{1 / 2}}\left\{k J_{m}^{\prime}(y)+\frac{m \mu}{y} J_{m}(y)\right\} \sin (m \phi+k z) \\
B_{\phi}^{m k}=\frac{-1}{\left(\mu^{2}-k^{2}\right)^{1 / 2}}\left\{k J_{m}^{\prime}(y)+\frac{m k}{y} J_{m}(y)\right\} \cos (m \phi+k z) \\
B_{z}^{m k}=J_{m}(y) \cos (m \phi+k z)
\end{gathered}
$$

where $y=r\left(\mu^{2}-k^{2}\right)^{1 / 2}$

The above solution is subject to the boundary condition $B_{r}(r=a)=0$ and must correspond to a given toroidal flux and total helicity. It should be noted that the $m=0, k=0$ term ( axisymmetric solution ) satisfies the boundary condition for all $\mu$ and gives rise to a finite toroidal flux. All other terms contribute zero toroidal flux and satisfy the boundary condition for discrete $\mu$. There are then two distinct types of solution. The first is the axisymmetric $(m=0, k=0)$ state which exists 
for a $\mu$ defined by the toroidal flux and total helicity. The second solution consists of the axisymmetric state, to give the required toroidal flux, plus contributions from the helical components. In this case the role of $\mu$ is to determine the amplitude of the helical deformation. It has been shown [5.4] that a minimum $\mu$ arises for $m=1, k a=1.25$ and is given by $\mu a=3.11$. It then follows that for $\mu a<3.11$, an axisymmetric profile is the relaxed state. Once this value is reached, the state will become helically deformed since the axisymmetric states give rise to a higher $\mu$. In this helical state, $\mu$ is independent of the total helicity for a given toroidal flux. At this critical value of $\mu$ the toroidal current reaches a limiting value. Any further increase in volt-seconds increases the helical deformation rather than the toroidal current. This remarkable feature of toroidal discharges is also found experimentally [1.11]. Figure 5.21 shows the results of two discharges where the system is driven to a high $\theta$. The high $\theta$ soon drops to a stable $\theta$ and remains there during the rest of the discharge.

The present theory can now be used to make comparisons with this remarkable result. For a square cross-section cylinder it can be shown [5.4] that the minimum value of $\mu$ corresponding to the onset of he.lical deformation is $5.6(\theta=1.4)$. The present theory disagrees with Taylor's theory by approximately a factor of two. A helical deformation is thus expected at $\theta \approx 3.0$.

Consider a $1 \times 1 \times 2$ rectangular cylinder represented by $10 \times 10 \times 20$ mesh points. An initial configuration which has an axial dependence is chosen, as in the test run case. Figures 5.22 (i) and 5.22 (ii) show the relaxed profiles obtained for an increase in driving field. As can be seen there is no helical deformation at the predicted $\theta$, in fact the toroidal current increases indefinitely. A plot of $|\mathbf{j} \times \mathbf{B}|_{\max }$ versus $\alpha$ (figure 5.23 ) shows that, for high $\theta$, the force-free constraint is violated. A higher resolution may thus be required to observe the possibility of current limitation and helical deformation. In figure 5.24 the same cylinder is 
represented by $20 \times 20 \times 40$ mesh points. Here, the force-free constraint is satisfied to the required order. Once again there is no helical deformation. There is the possibility that the system is rapidly seeking out an axisymmetric state and thus is unable to reach its lowest energy state. One way of overcoming this problem is to consider an axially dependent driving field. The results are shown in figures 5.25 (i) and 5.25 (ii). As can be seen, there is no current limitation and in fact there is little axial dependence. A second method is to consider a configuration which the axial flux has an axial dependence. This then forces the system to seek an axially dependent relaxed profile. It can be seen from figures 5.26 (i) and 5.26 (ii) that once again the results are disappointing. Figure 5.27 gives a high resolution result ( $20 \times 20 \times 40$ mesh points ) for the case where the axial flux is given an axial dependence. Figure 5.28 gives the the high resolution equilibrium profile for a $1 \times 1 \times 2 \pi$ rectangle. Once again there is no current limitation.

The non-appearance of a helical deformation could be due to a variety of reasons. The most obvious one is that theory developed for the $2 \mathrm{D}$ case is not extendable to $3 \mathrm{D}$ since the equivalence of anisotropic diffusion and resistive force-free evolution was proven only for the $2 \mathrm{D}$ case. A second reason could be due to the geometry being considered. It may be possible, but very unlikely, that the inclusion of toroidal effects could yield better results. A more likely reason could be the simple rectangular cross-section being considered. A circular cross-section may give more promising results.

Hitherto we have considered only rectangular cross-sections. If we consider solutions over a circular cross-section and which have only a radial dependence, the equilibrium state described by (1.14) can be written in simplified form as $\mathbf{j} \cdot \mathbf{B}=\alpha B_{z}$. This should be compared with $\mathbf{j} \cdot \mathbf{B}=\mu \mathbf{B} \cdot \mathbf{B}$ from Taylor's theory. The above constraint and the force-free constraint can be written as : 


$$
\begin{gathered}
j_{\theta} B_{z}+j_{z} B_{\theta}=\alpha B_{z} \\
j_{\theta} B_{z}-j_{z} B_{\theta}=0
\end{gathered}
$$

For the boundary conditions being considered, $B_{\theta}$ vanishes at the boundary and $j_{z}=\alpha$. It is therefore clear that the above two equations can only be satisfied, simultaneously, if $\alpha=0$. This leads to trivial equilibrium profiles. Now consider relaxing the force-free constraint and suppose that it zero only to order $\varepsilon$. Also, suppose that $\alpha$ is also of order $\varepsilon$. The equations, by choice, can then be written as :

$$
\begin{gathered}
j_{\theta} B_{z}+j_{z} B_{\theta}=\varepsilon \alpha B_{z} \\
j_{\theta} B_{z}-j_{z} B_{\theta}=-\varepsilon \alpha B_{\theta}
\end{gathered}
$$

This pair give the correct value of $j_{z}$ at the boundary. It is easy to show that they predict that $j_{z}=\varepsilon \alpha$ and $j_{\theta}=0$. In other words, we can obtain solutions where $B_{\theta}$ is proportional to the radial distance and is order $\varepsilon$ on the boundary, and where $B_{z}$ is everywhere constant. Although this solution satisfies both boundary conditions, to $\operatorname{order} \varepsilon$, it is very similar to the solution when $\alpha$ is set to zero for the exact forcefree case.

We have obtained solutions over a circular cross-section and this is exactly what we get. It should be noted that there is considerable difference to the square crosssection case. We have also considered an elliptical cross-section. For this case we get a numerical instability which grows with time. 


\section{Conclusions}

In the introduction we considered the evolution of a resistive plasma subject to force-free constraint. The evolution was considered in 2D which allowed the use of a stream function $\psi$. A diffusion equation was derivable, having no explicit velocity dependence, in terms of $\psi$ and $B_{z}$. Any solutions of this evolution equation was subject to the force-free constraint. We next considered the evolution of magnetic fields in an anisotropic static conductor and found that the resulting evolution equation was identical to that describing the diffusion of magnetic field lines in a resistive plasma. Describing the anisotropic diffusion process is a much simpler mathematical problem and it was therefore considered in subsequent chapters.

In the second chapter we considered the numerical solution of the evolution equations using the explicit finite difference method. We found that the boundary conditions on $\psi$ could be implemented in a variety of ways and we considered two. We also found two methods of implementing the axial flux constraint. The first involved writing the difference equation in a conservative type form and the second involved a simple scaling. All the above methods were implemented and the codes were found to give very good agreement. We next considered solving the problem using all the components of $\mathbf{B}$ with the hope that the methods would be extendable to $3 \mathrm{D}$. We found two methods of implementing the divergence-free constraint. The first involved using $\nabla \cdot \mathbf{B}=0$. Here, an evolution equation was compromised in favour of the constraint and the updating was done using $\nabla \cdot \mathbf{B}=0$ itself. The second method used the symmetry properties of mixed derivatives. The difference equations were chosen such that the constraint was satisfied automatically. The above methods were implemented and the codes once again gave very good agreement. The methods were extendable to $3 \mathrm{D}$ and two codes were written incorporating the two methods of ensuring $\nabla \cdot \mathbf{B}=0$. Initially we considered a model which allowed tangential currents to flow at a boundary and it was this model which gave good 
agreement between the codes. We next insisted on the vanishing of the surface currents and found that all codes, except one, failed. This was where the divergence-free constraint was used to ensure that the constraint remains satisfied. This highlighted the importance of considering as many approaches as possible in obtaining numerical solutions.

In the third chapter we considered obtaining solutions of arbitrary cross-sections. Law

We found that Ohm's/gave a boundary condition for the normal derivative of $B_{z}$ and a boundary condition which coupled the derivatives of $B_{x}$ and $B_{y}$. We found that by taking forward/backward difference approximations into the solid we could obtain a remarkably simple equation which would give the unknown boundary values of $\mathbf{B}$. The flux-conservation scheme employed in the rectangular crosssection case was found to be extendable to arbitrary cross-sections.

In chapter 4 we were able to generalise the rectangular cross-section codes to incorporate toroidal effects. Once again a stream function and also all the components of B was used for obtaining the required solutions. The methods were extendable to 3D.

Altogether then, the suite of codes totaled approximately 20,000 lines of source. The variety of geometries used justified the use of explicit schemes. The implicit schemes would have just taken too long to incorporate in each code due to the number of codes written. The explicit nature did however restrict the size of time step used. All codes were run on a CRAY XMP/416 which has four processors ( 250 Mflops each ) and which supports vector processing. A single processor was used for all runs as otherwise each code had to be multitasked which would have been time consuming. All codes have been vectorised with a typical increase in speed of a factor of two or three. For the square cross-section case, represented by $10 \times 10$ mesh points, we used a mesh spacing of $1.0 / 9.0$ and time step $\Delta t=2.5^{-3}$. 
The compilation ( $=1,500$ lines of source ) took 14.3 secs. and the execution ( CPU ) time being only 3.6 secs. For $20 \times 20$ mesh points, the execution time increased to 40.6 secs.

In the final chapter we presented a variety of results. We initially observed the two time scales associated with the two very different conductivities. By considering the value of $\mathbf{j} \times \mathbf{B}$ at a boundary, the inconsistency, introduced by the driving field was found to have little effect on the force-free evolution of the plasma provided its magnitude was chosen with care.

We next obtained a selection of $F-\theta$ co-ordinates, for the $2 \mathrm{D}$ square cross-section case, for a variety of axial fluxes and three different values of driving field. All the points were found to lie on a single curve. This was in agreement with Taylor's theory and experimental data. The field reversal value was found to be $\theta \approx 2.0$. This was nearly a factor of two out compared with Taylor's theory which predicts a value of $\theta \approx 1.1$. The profile was compared with that of another simulation and better agreement was found. The discrepancy was attributed to the unrealistic approximation of constant resistivity. A variety of other universal curves were generated and from these it could be concluded that the final relaxed state is completely defined once the axial flux and driving term are specified. In Taylor's theory, the driving term is proportional to the global helicity, at constant axial flux, and the final state is completely defined once the global helicity and axial flux are prescribed. The present theory thus agrees well with Taylor's theory on this point.

Another interesting profile was obtained for a axial current versus axial flux plot. We found that at a certain value of axial flux there exist states which are inaccessible by the plasma. About this critical value there are two modes at which the plasma can be driven, one at high current and one at low current. These modes are also found to exist experimentally. The existence of degenerate states were also 
found by plotting magnetic energy curves.

By considering a $1 \times 2$ rectangle, we were able to show that the corresponding $F \theta$ profile was only slightly different to the square cross-section case. This also agrees well with Taylor's theory.

We initially considered a model shere surface currents were allowed to flow and this gave field reversed states. When no boundary currents were allowed to flow, no field reversal was obtained and the $F-\theta$ profile was very similar to that predicted by the force-free paramagnetic model.

Once toroidal effects were taken into account we obtained three $F-\theta$ profiles for aspect-ratio 2.0, 4.0 and 10.0. The curvature was seen to have very little effect on the profile, once again agreeing with Taylor's theory and experiment. The critical flux value was found to be smallest for the smallest aspect-ratio but was higher for 4.0 than 10.0 . The critical value was therefore found to increase with aspect-ratio, reach a maximum and then fall to a finite value. The maximum occurred at an aspect-ratio of $\approx 3.0$. This is the most suitable value to build toroidal devices since this gives the highest current before reaching the critical flux value, beyond which the maximum current is much lower.

The results generated by the $3 \mathrm{D}$ code were found to be very disappointing for high $\theta$ as only axisymmetric solutions were generated. Although the 3D code should generate axisymmetric profiles, it should also show the onset of helical deformation at a critical $\theta$. This was not observed. A full 3D toroidal code has also been developed. Here, the divergence-free constraint was satisfied using the symmetry properties of mixed derivatives. This method fails when considering a current free surface model and so no results were presented. 
We finally considered solutions over a circular cross-section. The only solutions we could obtain were very trivial which did not possess any real structure. When an elliptical cross-section was considered, the solution was found to become unstable. The instability was found to grow in time and so was explosive in nature.

\section{Future work}

If we use the MHD equations, solutions to the force-free problem could only be obtained by considering non-ideal boundary conditions or by relaxing the forcefree constraint by, for example, including a pressure term. It would be interesting to see if such changes yielded results different to those presented. The inclusion of a structured resistivity would eventually have to be considered as experimentally it is found not to be constant.

The 3D solutions did not predict helically deformed states and so it must be decided if the equivalence that was proven is extendable to 3D. What could be done is to consider a non-rectangular cross-section and see if results are any promising. This means that the curved boundary code has to be considered and the numerical instability that arises has first got to be removed. Toroidal effects could also be taken into account then. 


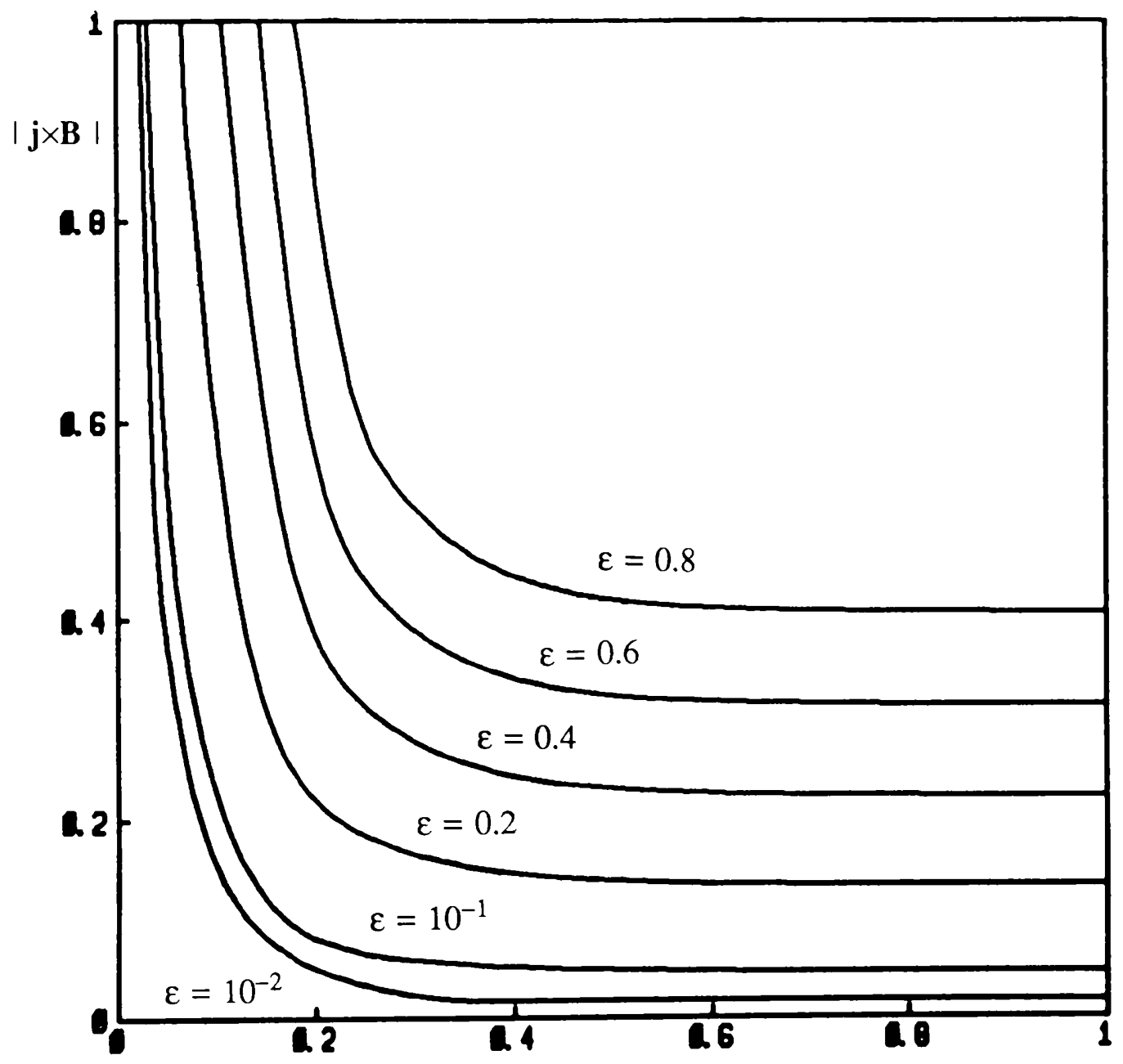

normalised time

Figure 5.1

Time development of $|\mathbf{j} \times \mathbf{B}|_{\max }$ for varying values of $\varepsilon$. The time taken to reach a relaxed state has been normalised to unity. By noting the rapid drop in gradients the two time scales associated with the conductivities parallel and perpendicular to the magnetic field are clearly visible. 


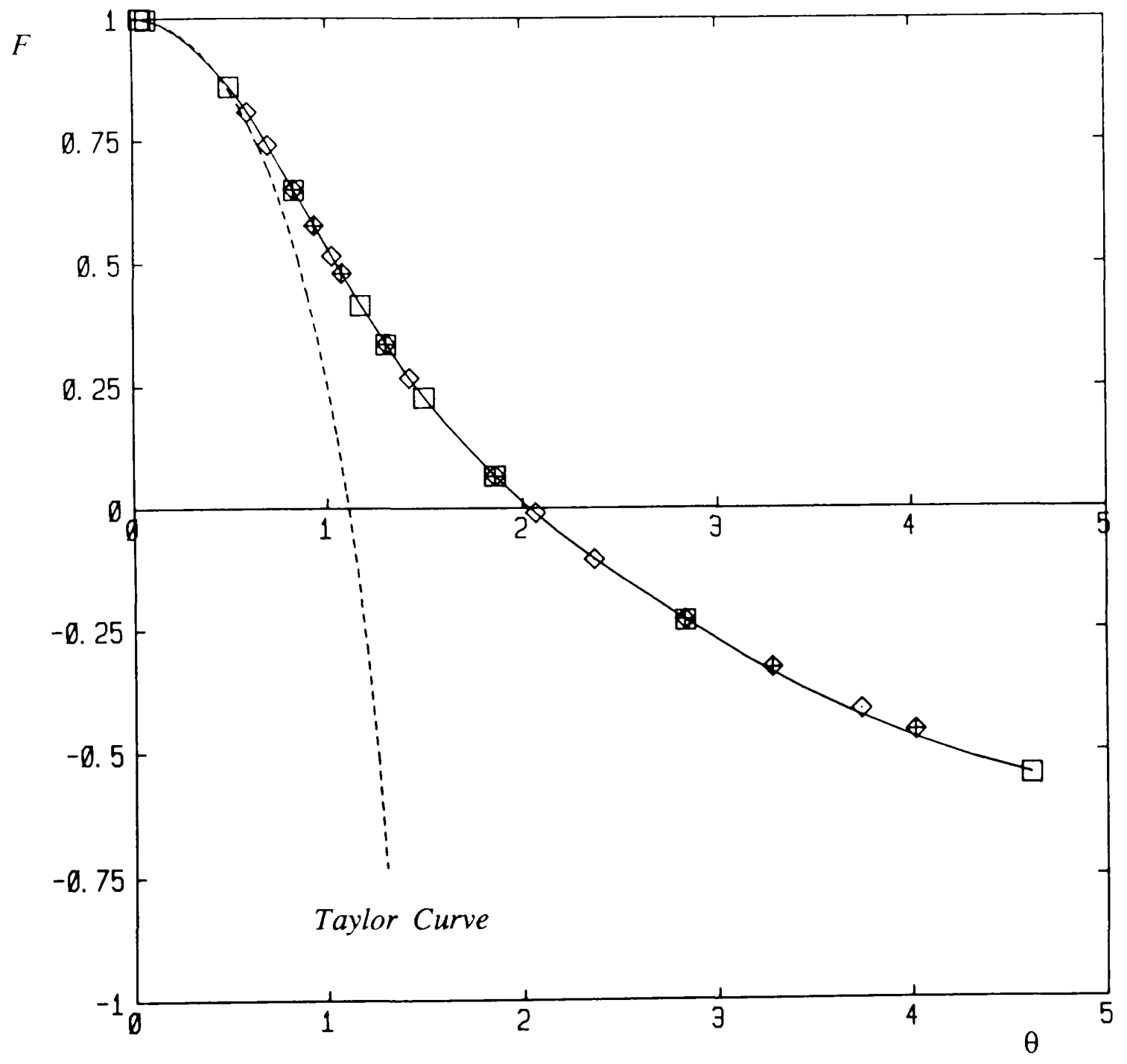

Figure 5.2

Universal $F$ versus $\theta$ profile obtained by varying the axial flux and using three different values of driving field ( $\alpha=0.1,0.3$, and 0.5 ). All points are clearly seen to lie on a single curve. Also shown is the result predicted by Taylor's theory. 


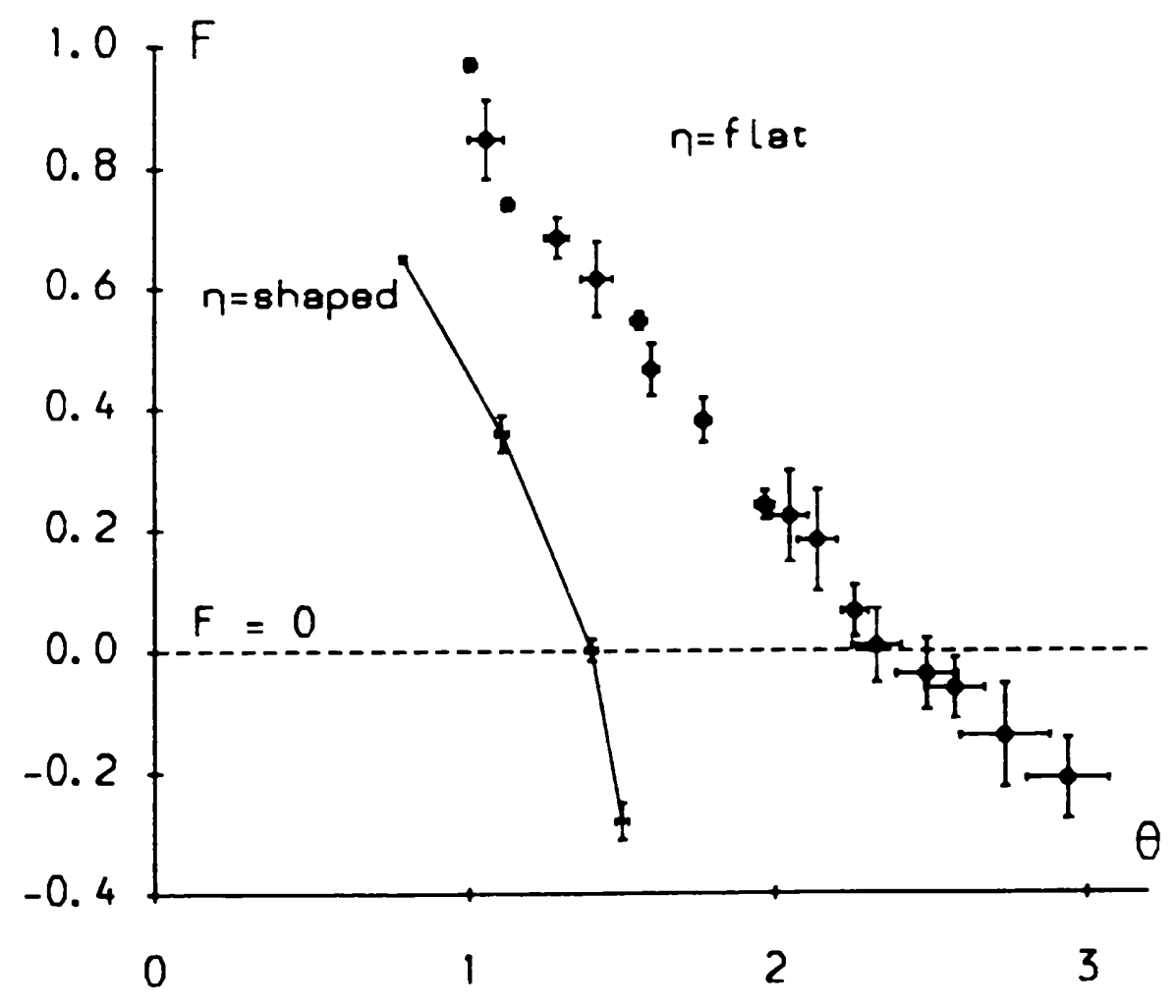

figure 5.2b

Universal $F$ versus $\theta$ profile ( from Kirby, 1988 ) generated using the MHD equations for a circular cross-section. Choosing a structured resistivity ( $\eta=$ shaped ) can be seen to give a profile in better agreement with experiment. 
Three typical profiles of $\mu=\mathbf{j} \cdot \mathbf{B} / B^{2}$ across the center of a square cross-section.

Profile of $\mu$ obtained by Kirby ( 1988 ) together with an experimental result. The results are for a circular cross-section. 


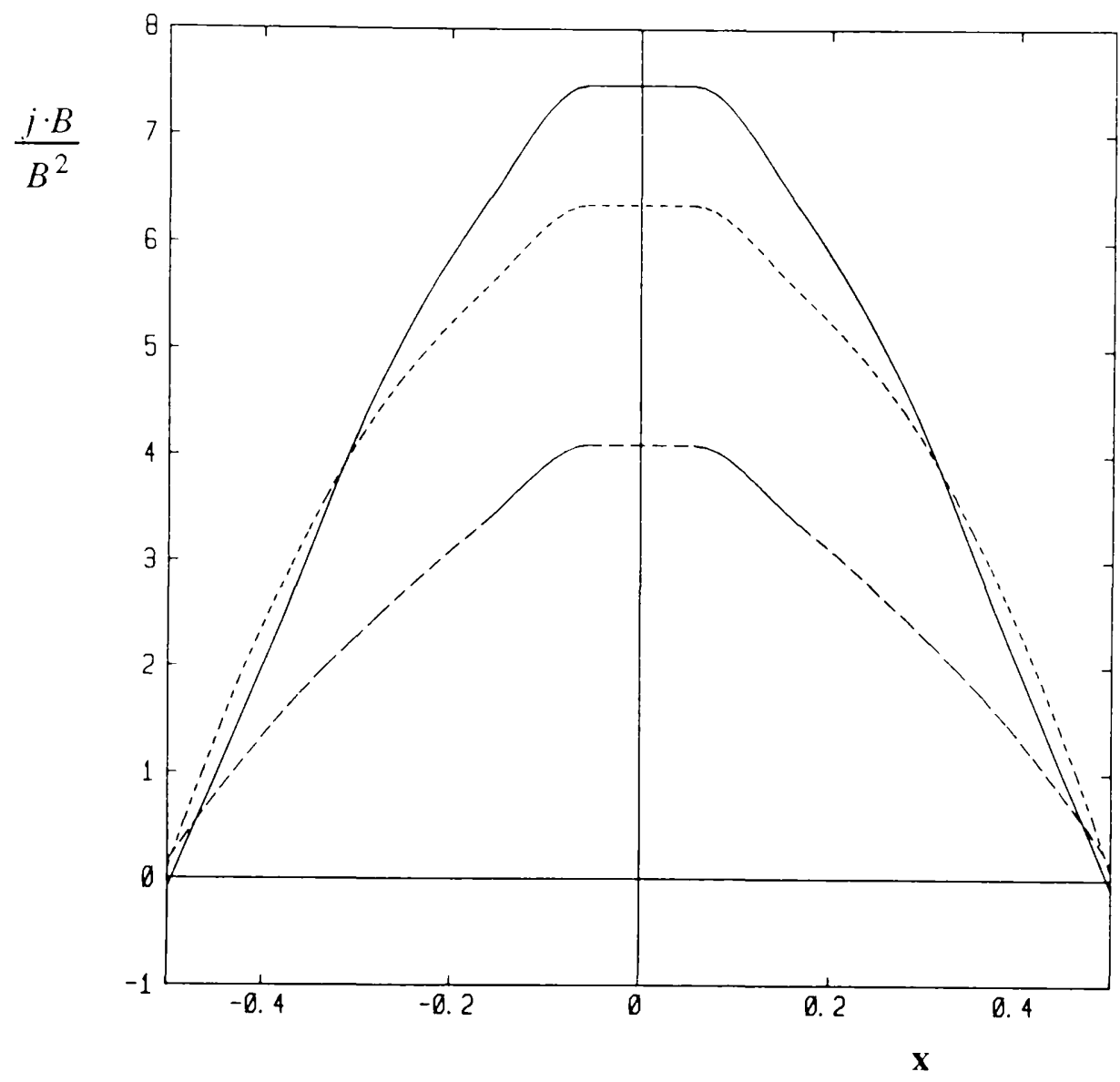

Figure 5.2c

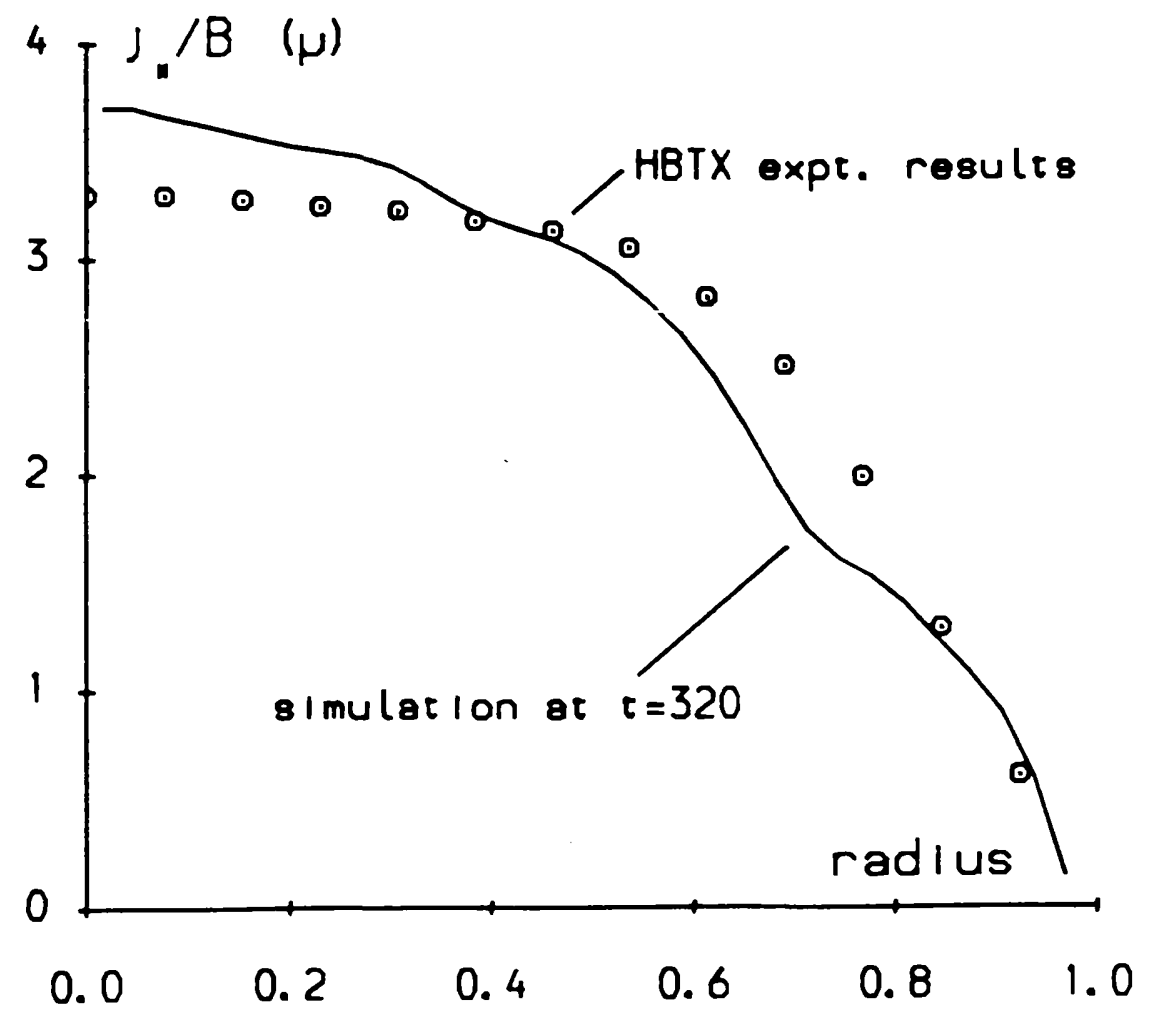




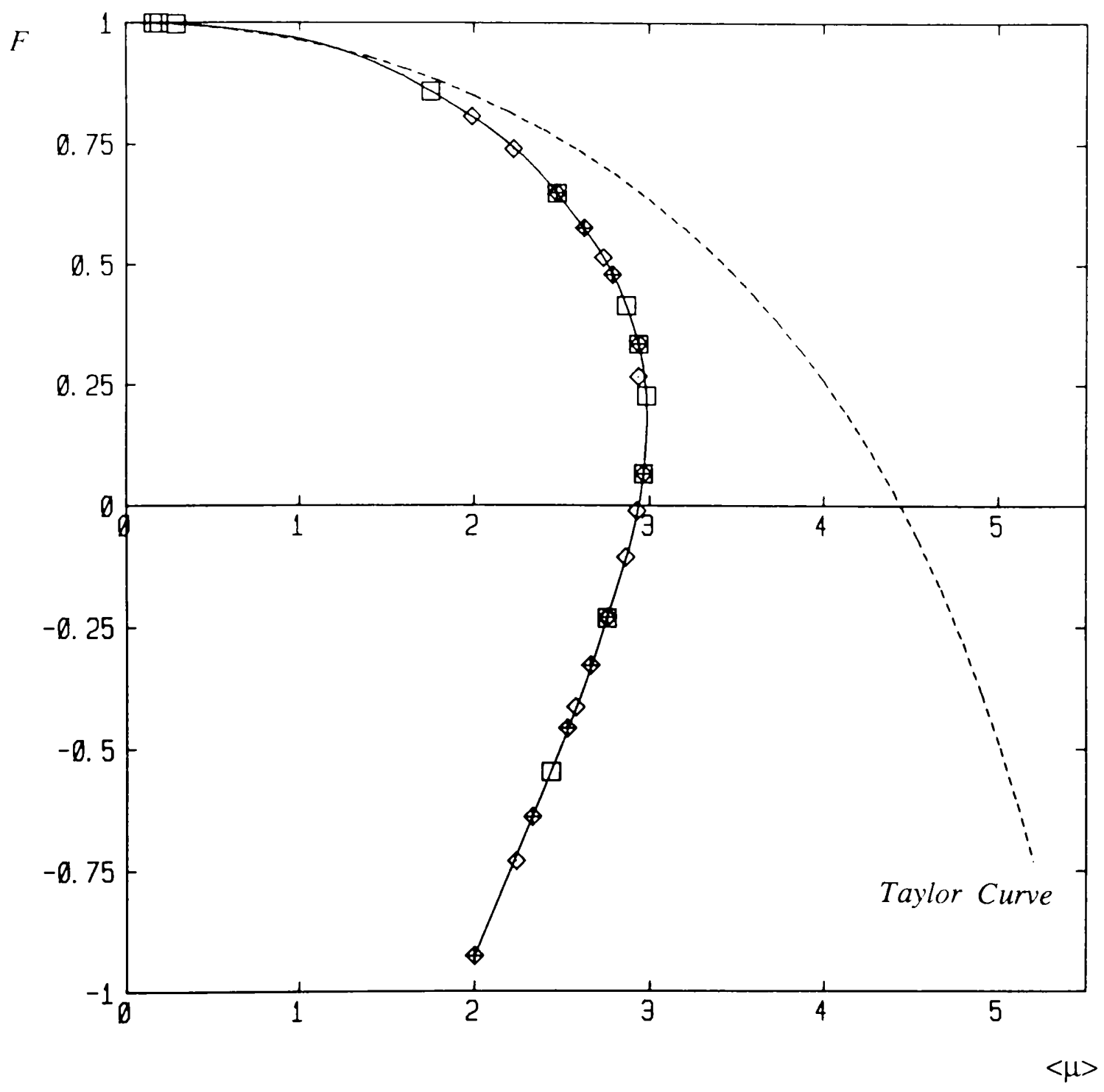

Figure 5.3

Universal $F$ versus $\langle\mu\rangle$ profile together with the result predicted by Taylor's theory. 


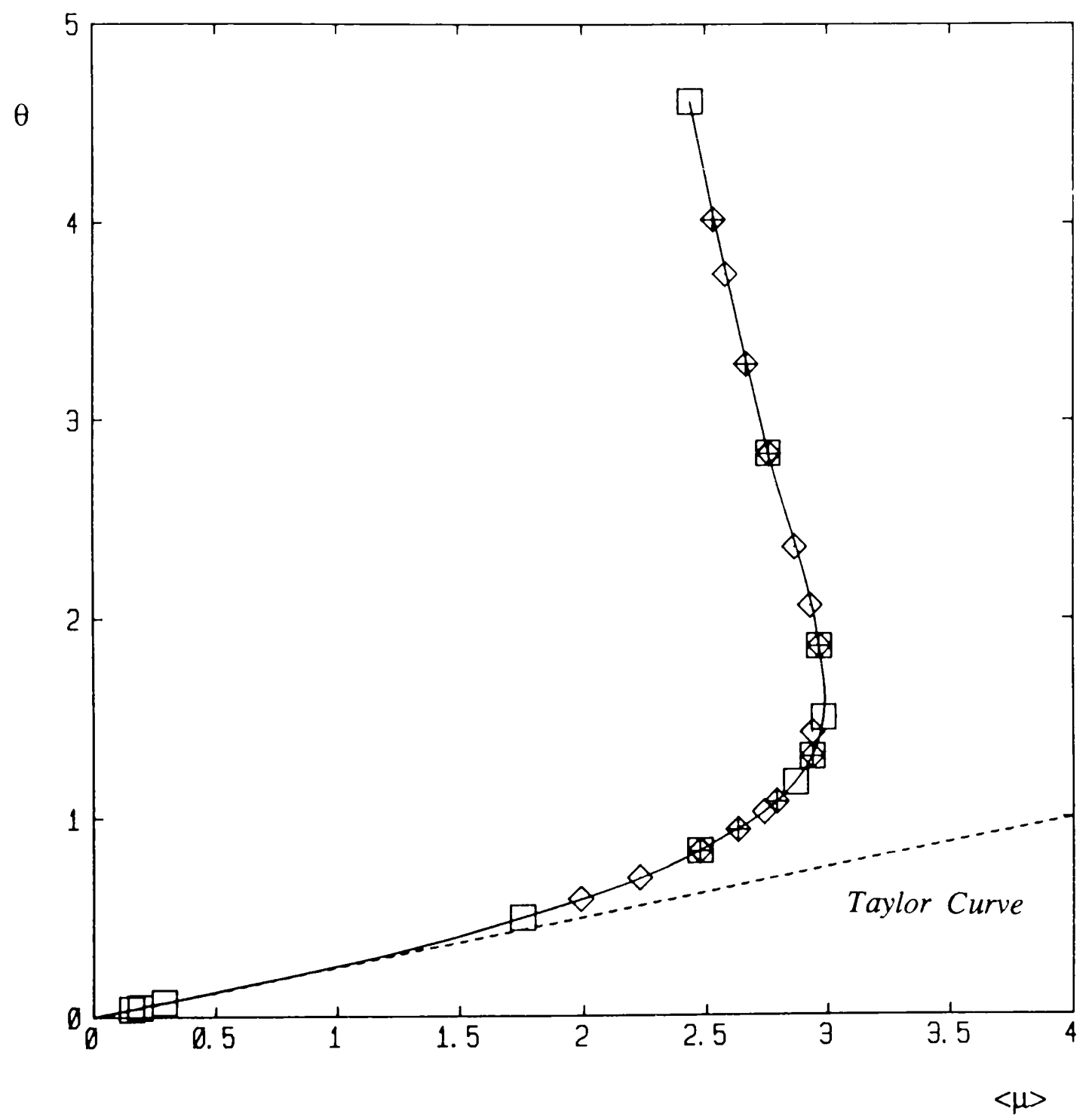

Figure 5.4

Universal $\theta$ versus $\langle\mu\rangle$ profile reveals linear relationship only for small $\theta$. 


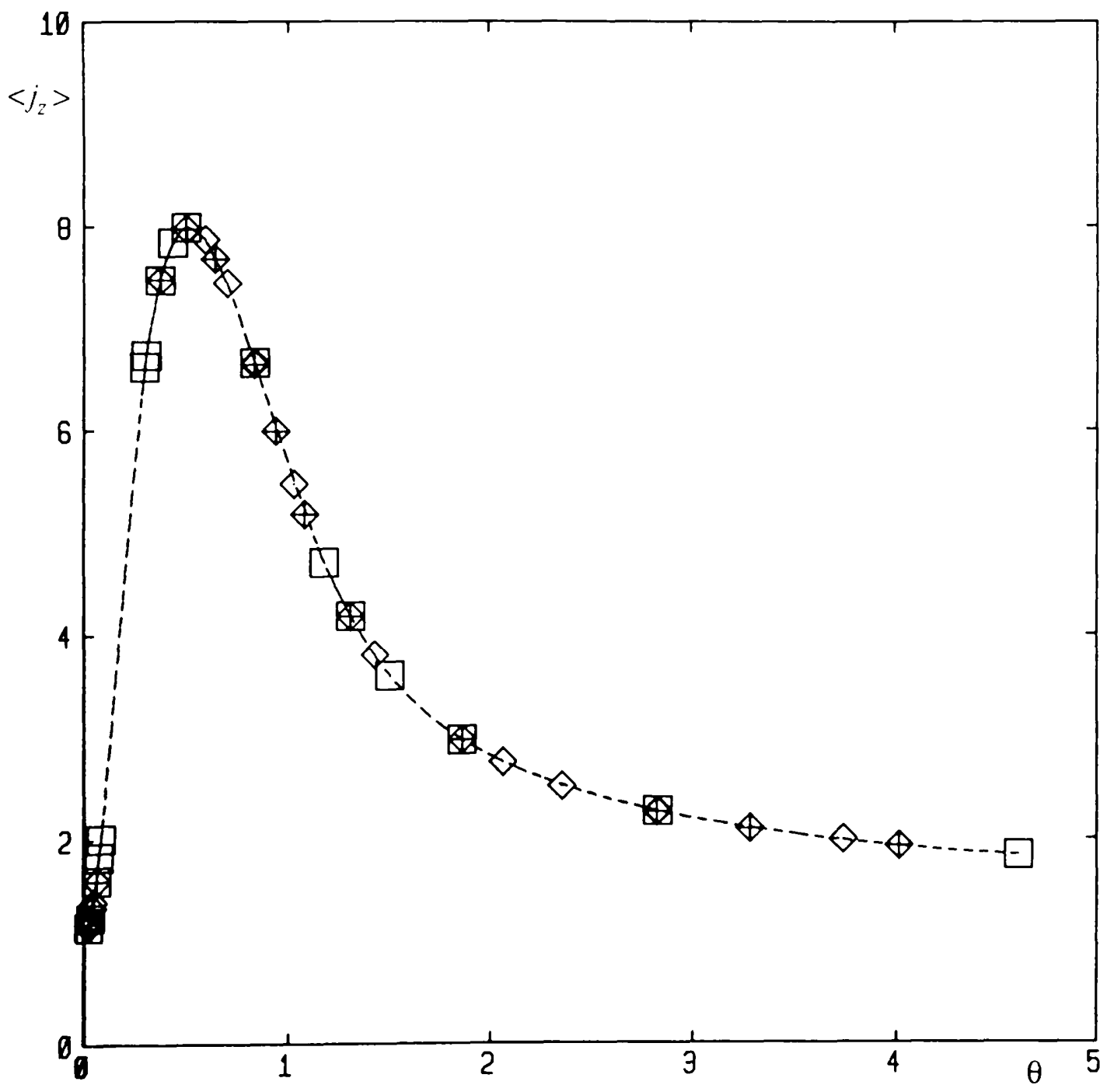

Figure 5.5

Universal profile showing the toroidal current is proportional to the driving field. A limit on the toroidal current, for fixed $\alpha$, can also be seen. 


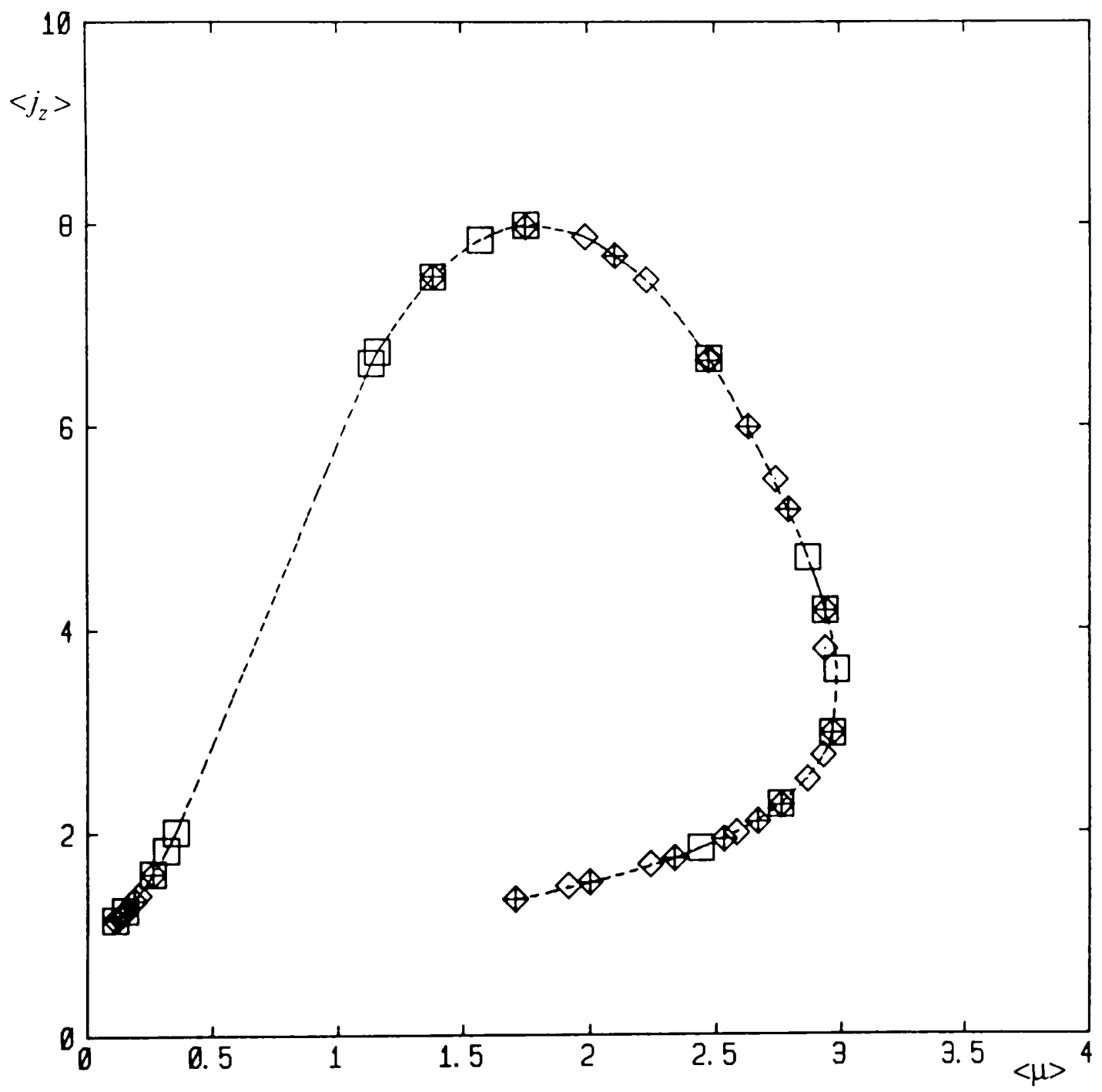

Figure 5.6

A plot of $\left\langle j_{z}>/ \alpha\right.$ versus $\langle\mu>$ reveals upper limits on both the current density and $<\mu>$. 


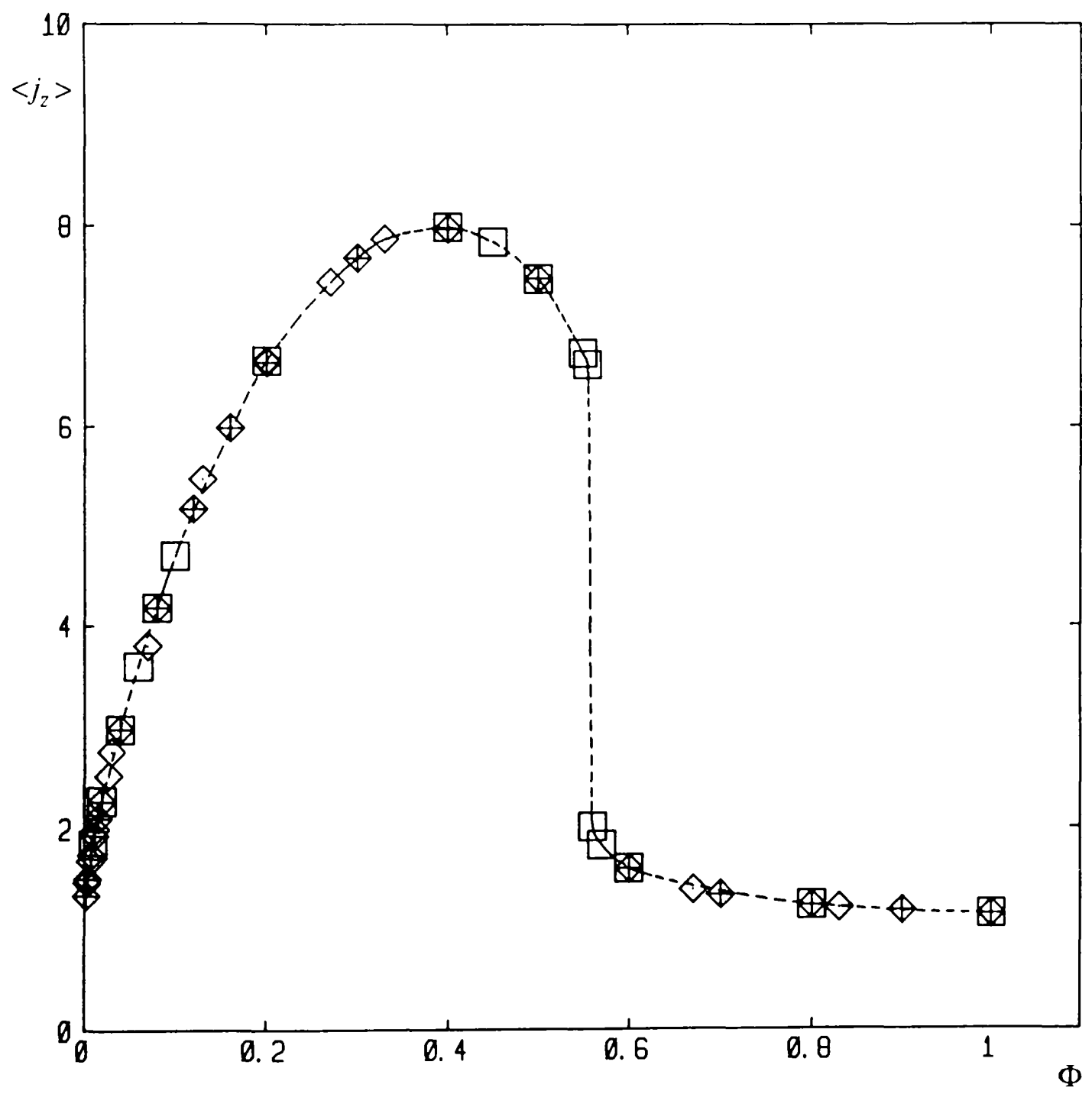

Figure 5.7

At a critical axial flux there exist inaccessible states. About this value the system can be driven at high or low current mode. 
Time development of $j_{2}, \theta$ and $F$ for a case where there is a rapid rise in toroidal current. 
$\left\langle j_{2}>\right.$ 0. $6-$

อ. $5-$

e 4 -

อ. $3-$

อ. 2

D. 1

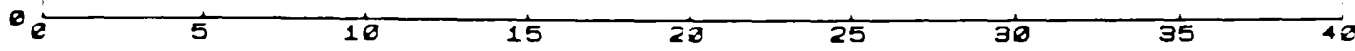

time steps
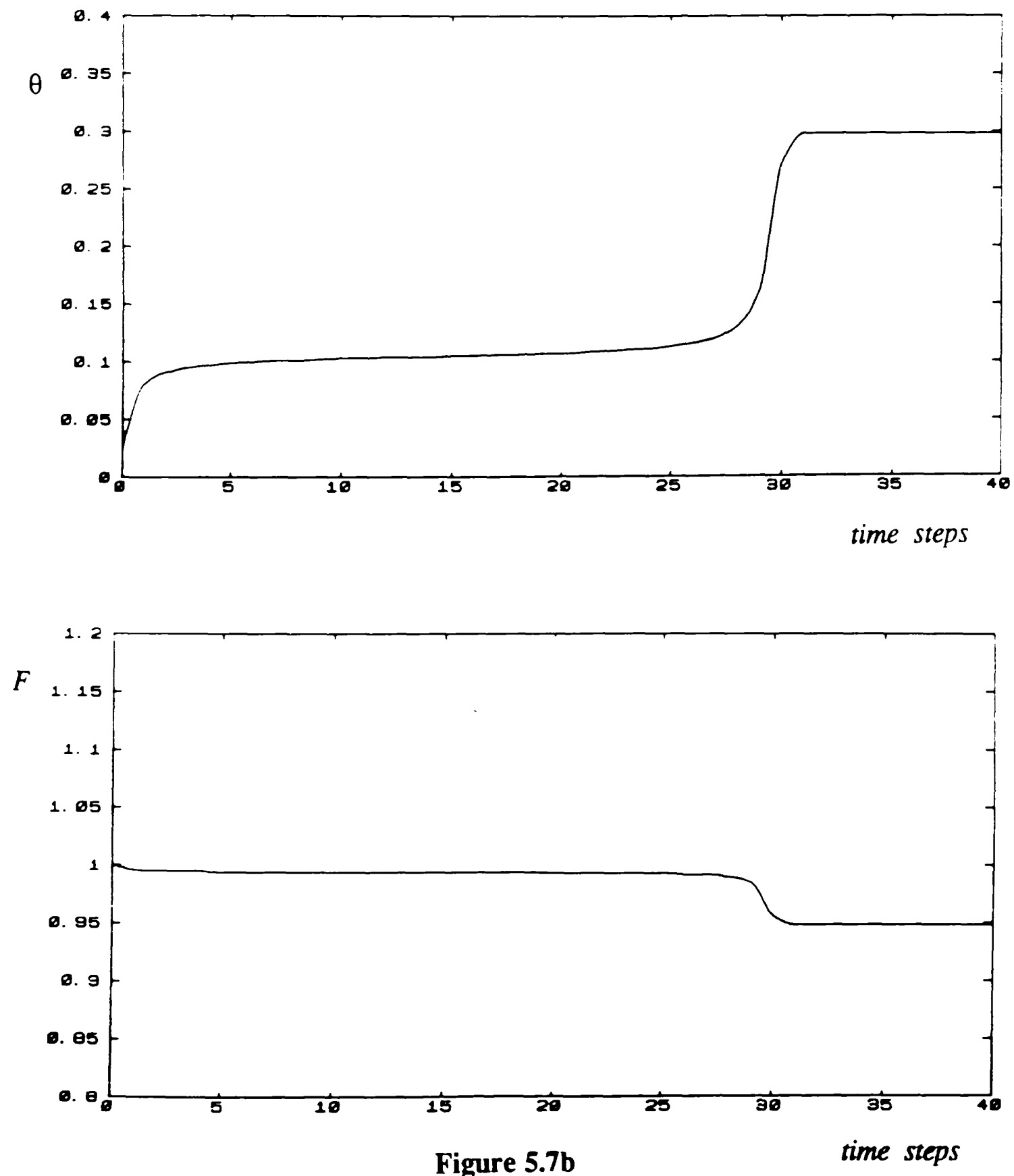


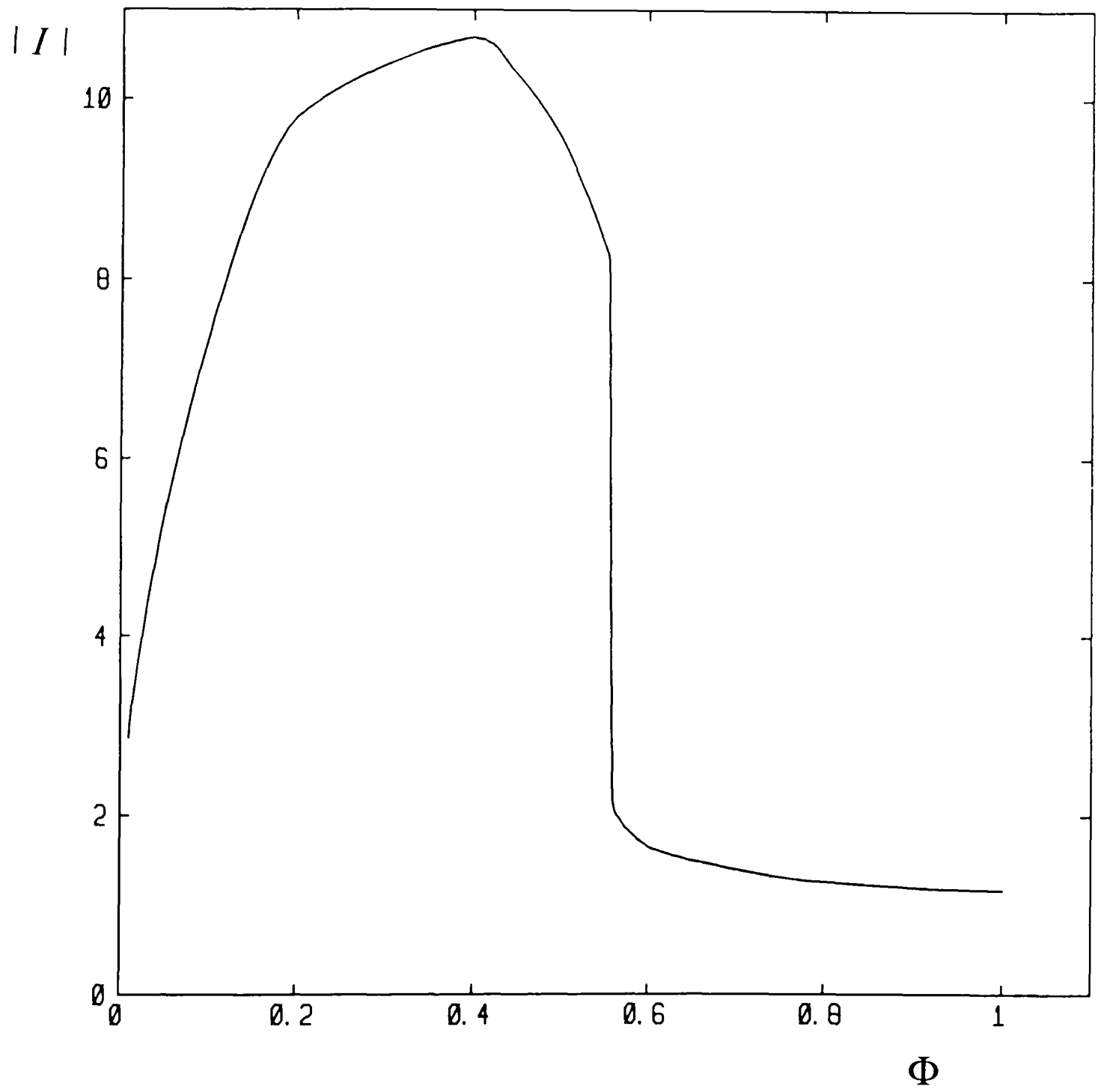

Figure 5.7c

A plot of total current against axial flux reveals that it is not only the axial current but the total current that may rise or fall significantly. 


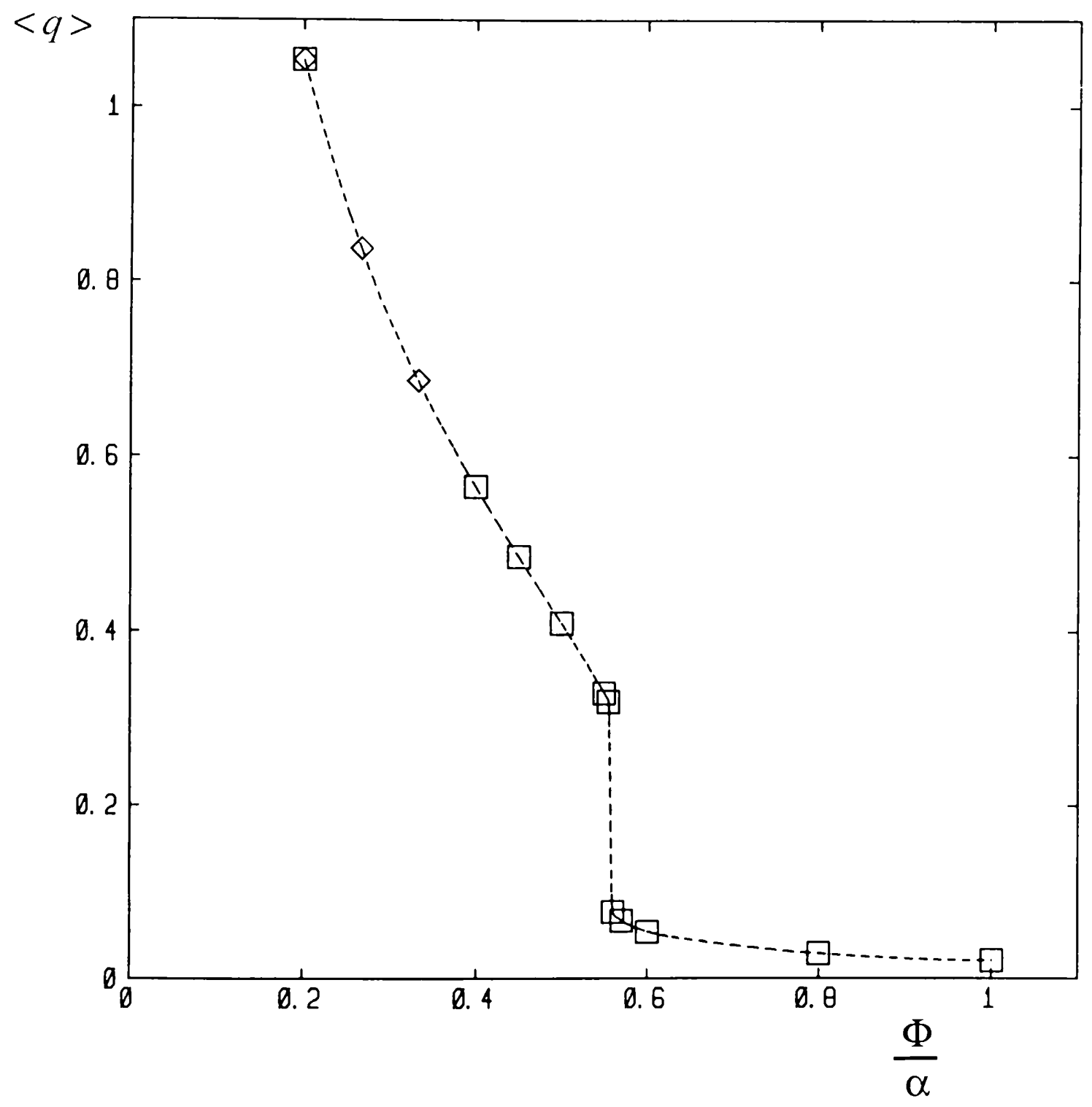

Figure 5.7d

One choice of definition of the safety factor $q$ reveals a universal profile. 


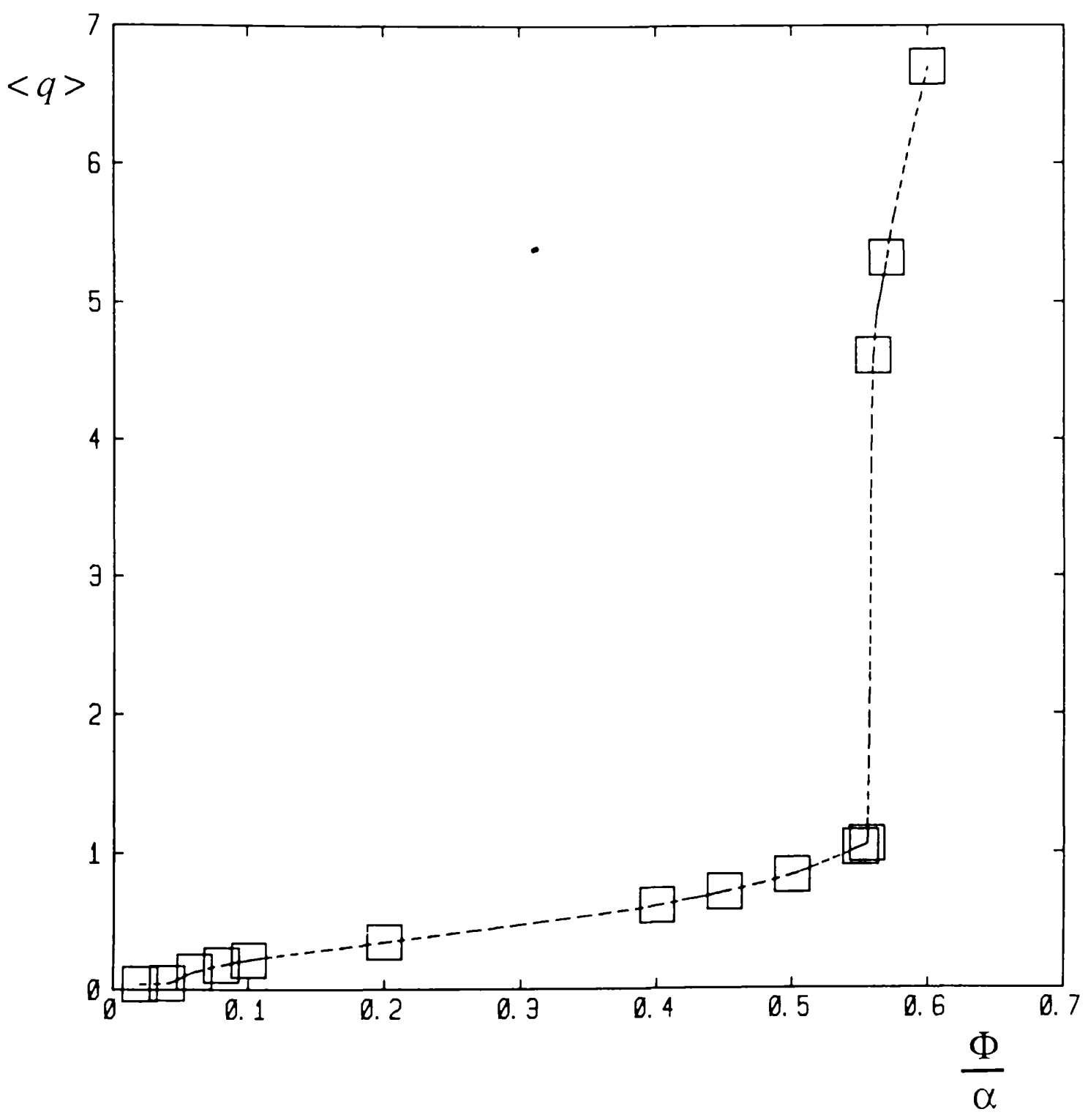

Figure 5.7e

Second choice of definition of the safety factor $q$ reveals a universal profile although only one set of data is shown. 


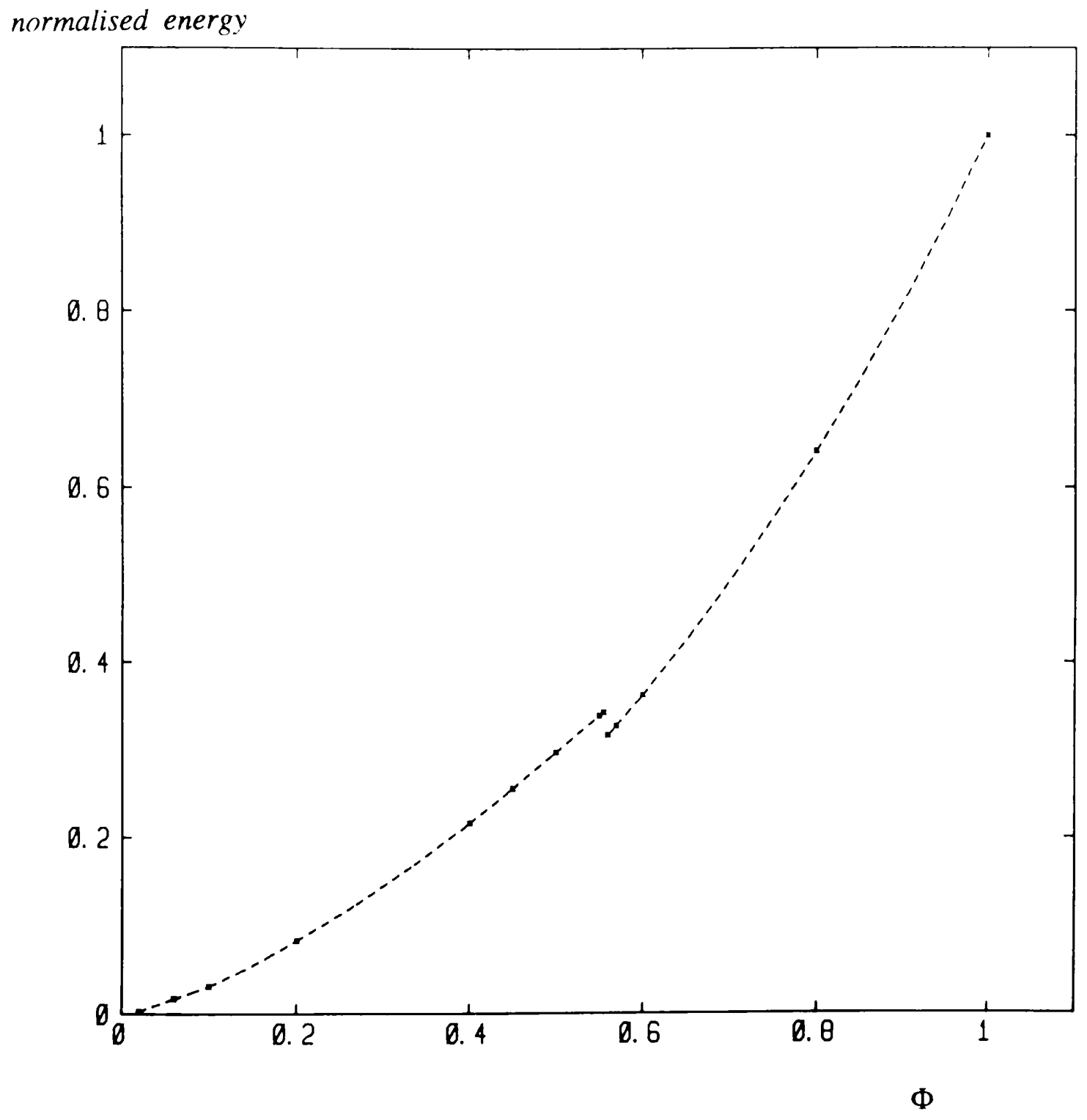

Figure 5.8

Energy curve showing the existence of degenerate states and discontinuous relationship with axial flux. 


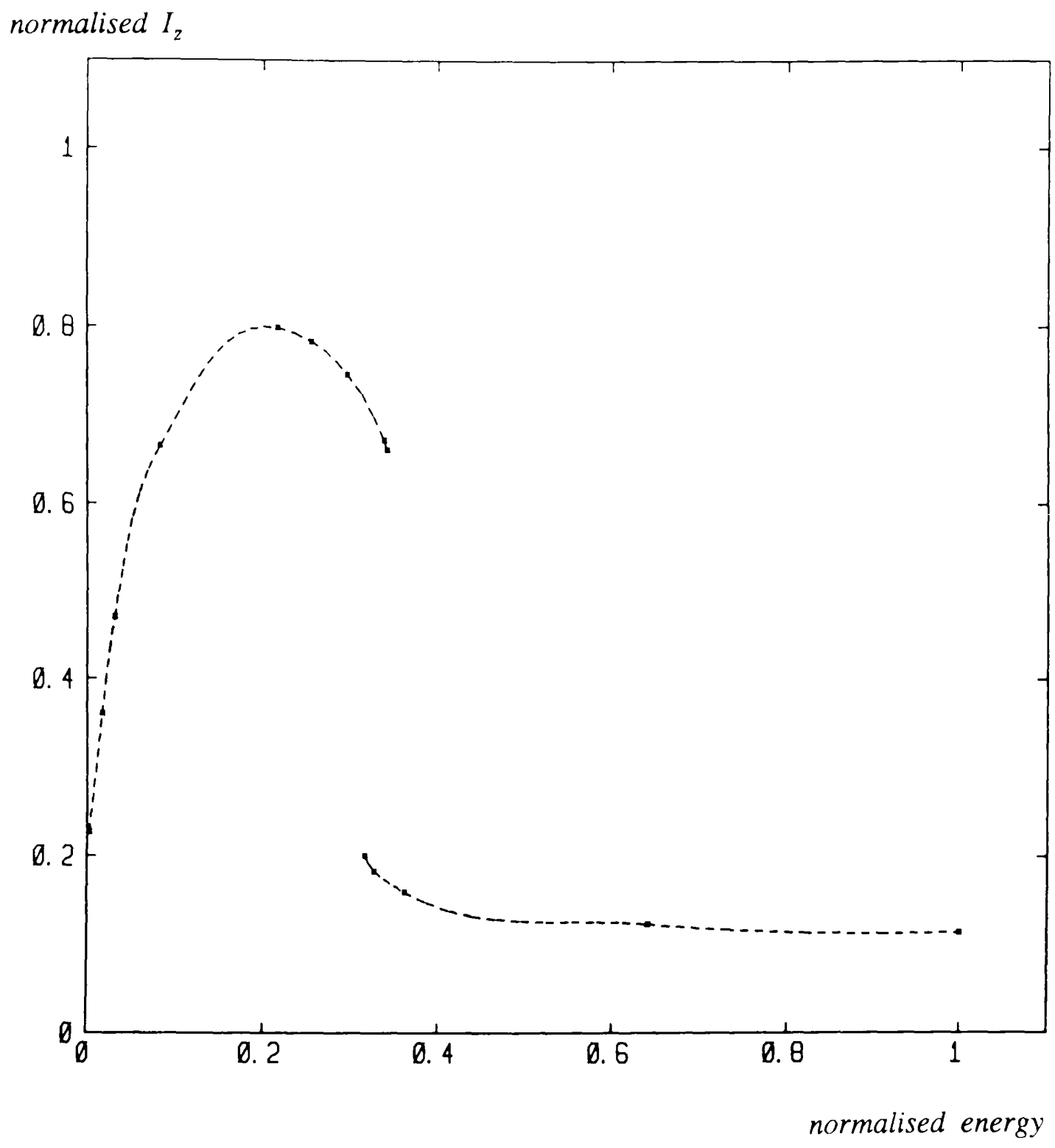

Figure 5.9

Toroidal current curve showing the existence of degenerate states and discontinuous relationship with magnetic energy. 


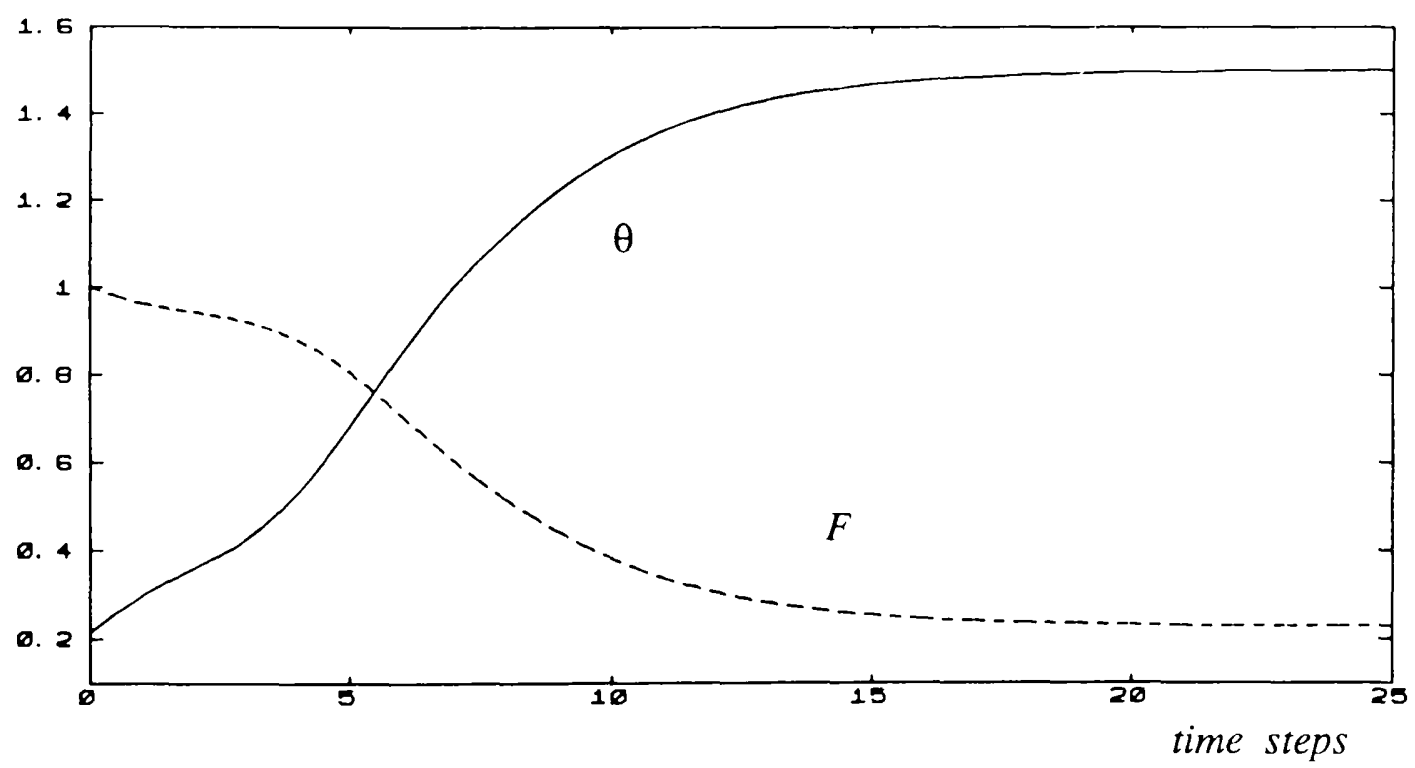

Figure 5.10

Time development of $F$ and $\theta$ for the case where the final state has no field reversal.

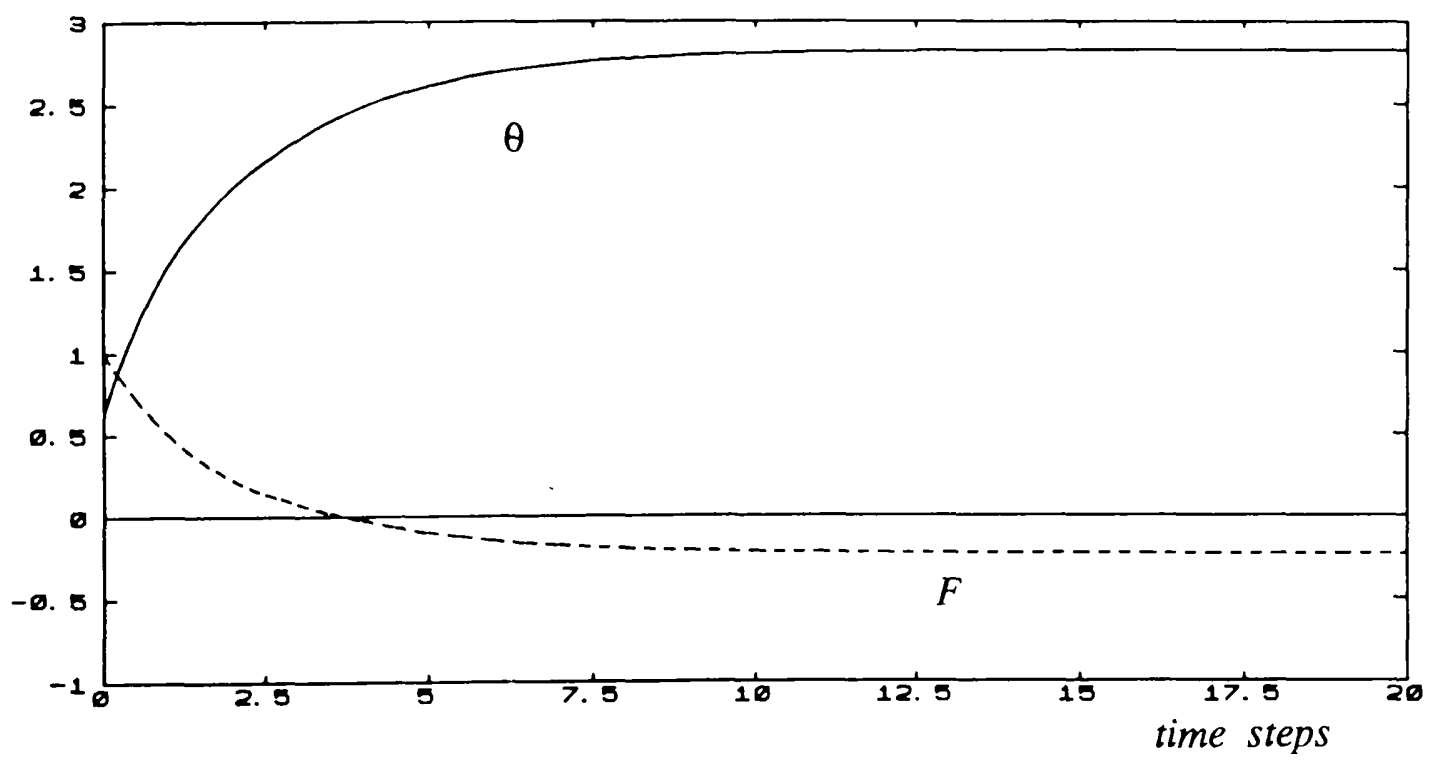

Figure 5.11

Time development of $F$ and $\theta$ for the case where the final state has field reversal. 

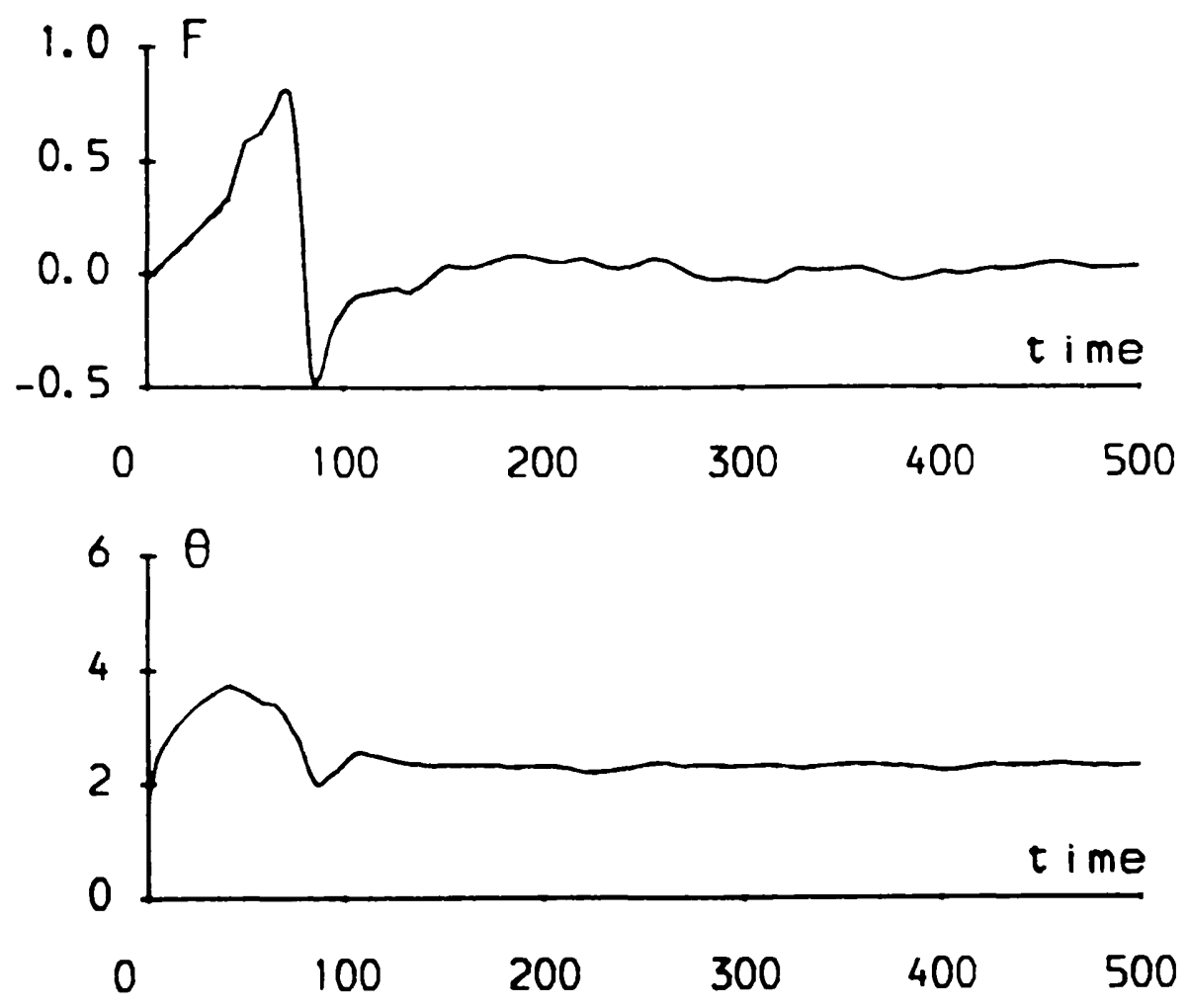

Figure 5.11b

Time development of $F$ and $\theta$ for the case where the final state has field reversal obtained by Kirby ( 1988 ). 


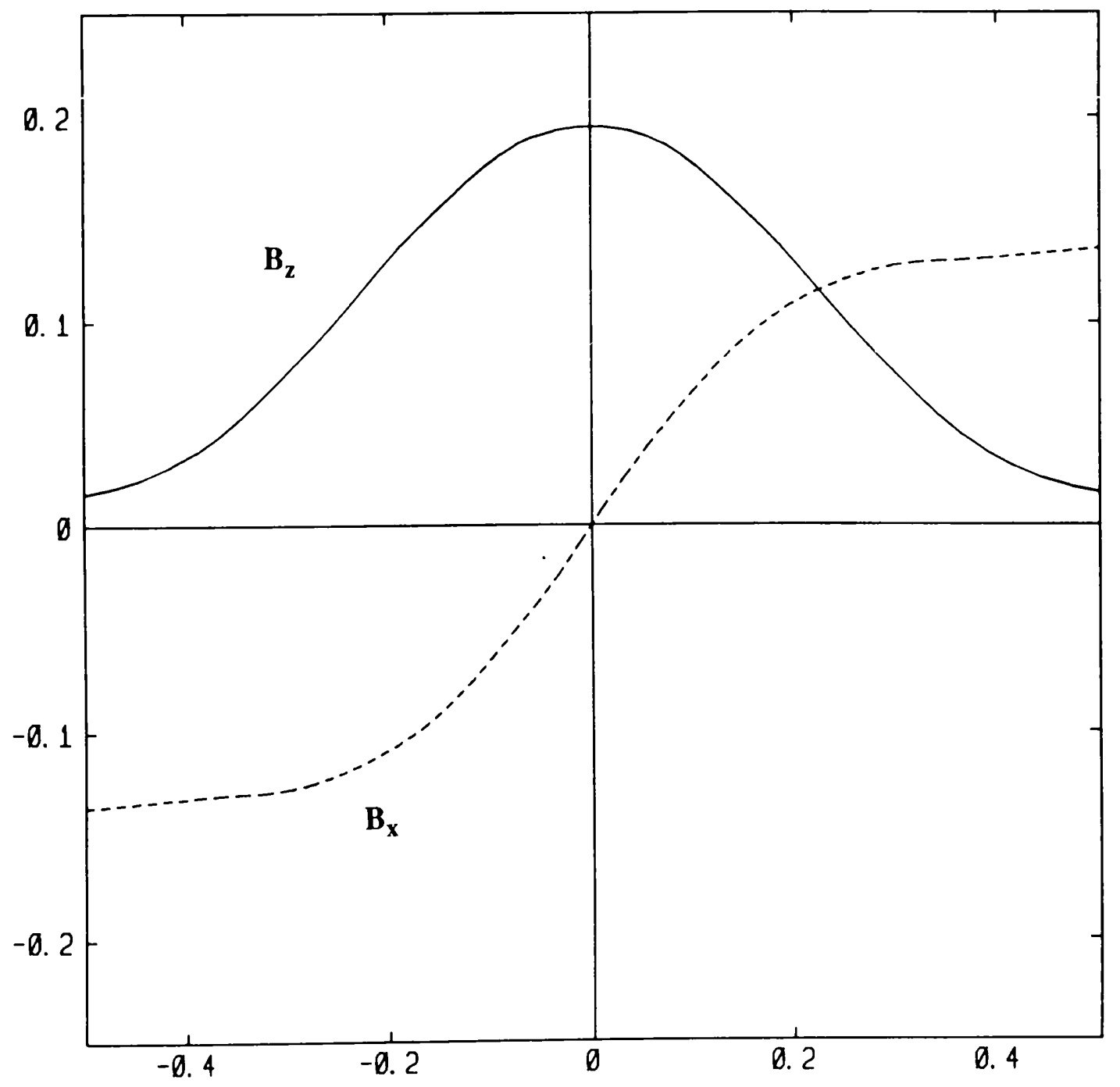

Figure 5.12

Typical profiles of $B_{z}$ and $B_{x}$ for the square cross-section case and where there is no field reversal. 


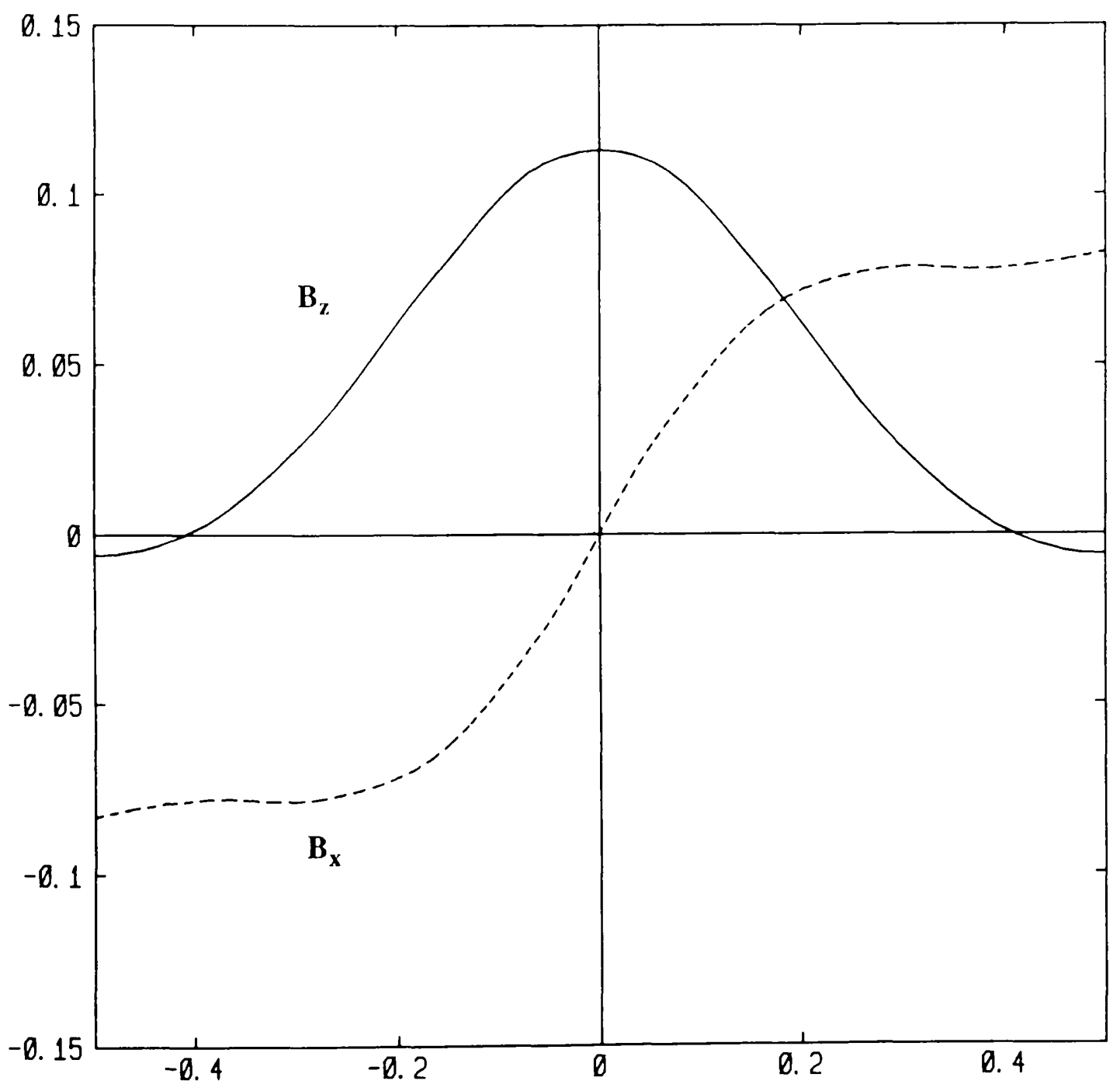

Figure 5.13

Typical profiles of $B_{z}$ and $B_{x}$ for the square cross-section case and where there is field reversal. 


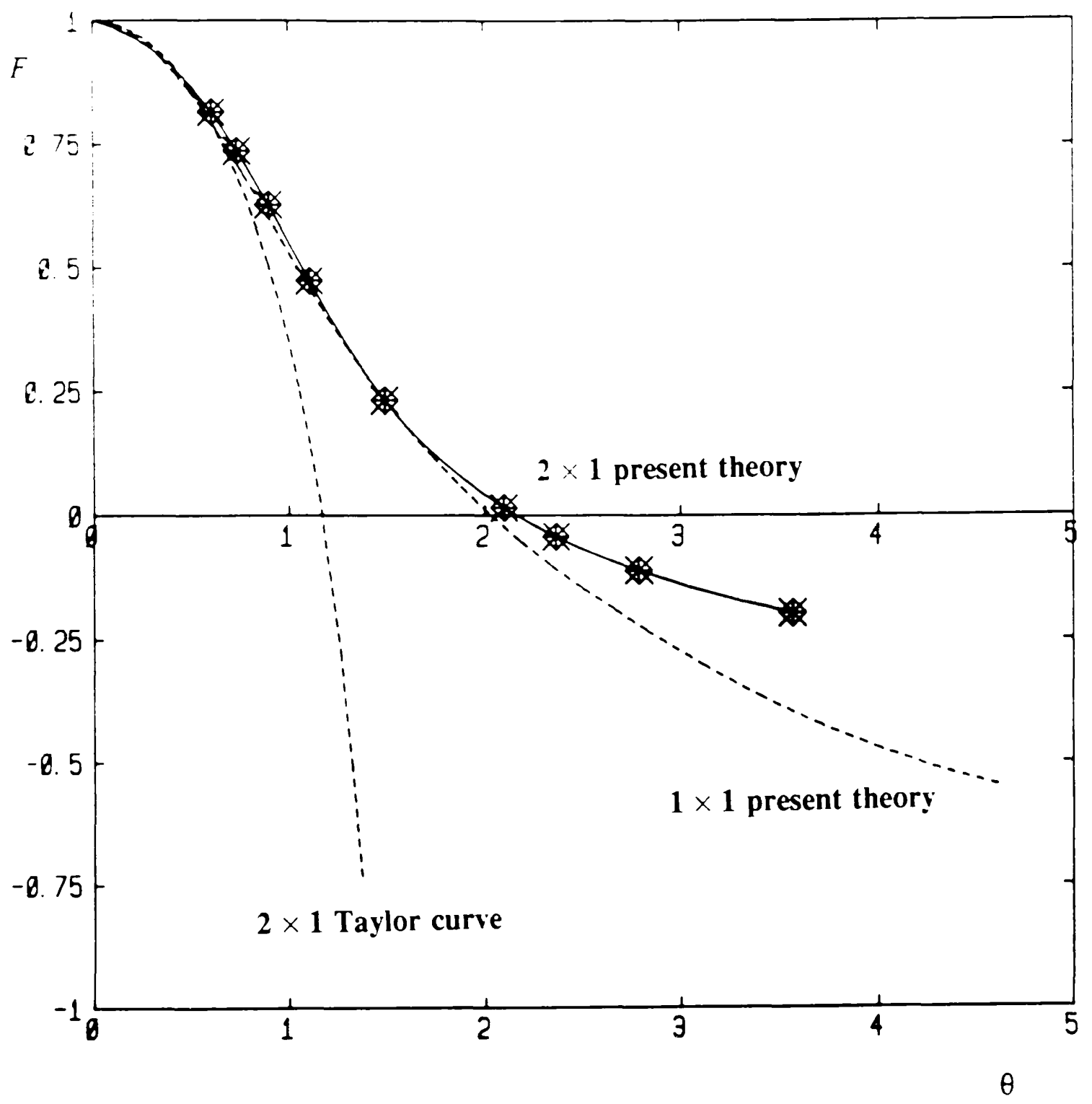

Figure 5.14

$F$ versus $\theta$ profile for a $1 \times 2$ rectangle indicates that the dimensions of the rectangle has little effect on the field reversal value. Also shown is the result predicted by Taylor's theory. 


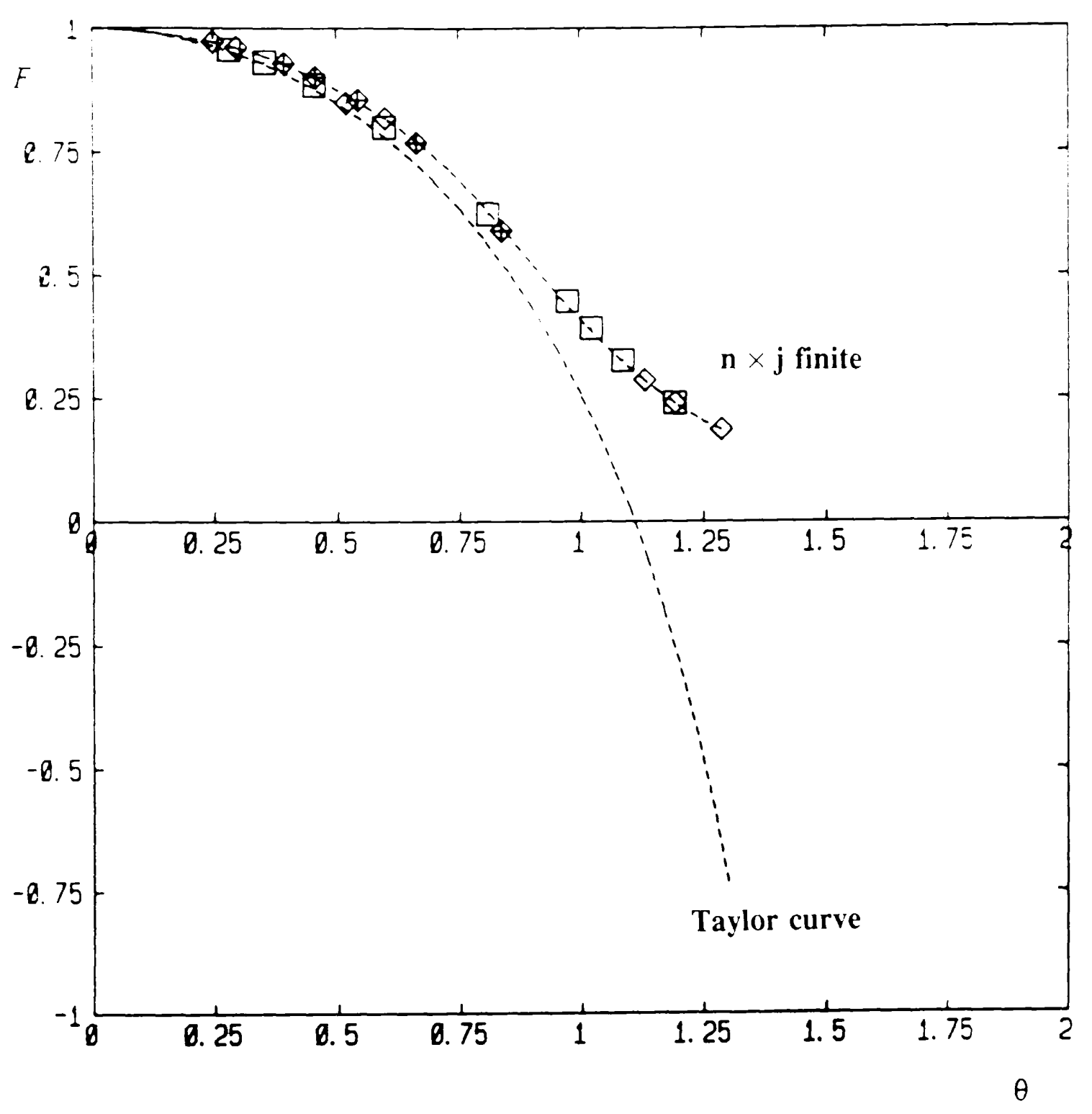

Figure 5.15

$F$ versus $\theta$ profile for a square cross-section and where tangential currents at the boundary are allowed to flow. No field reversed states are obtained. 

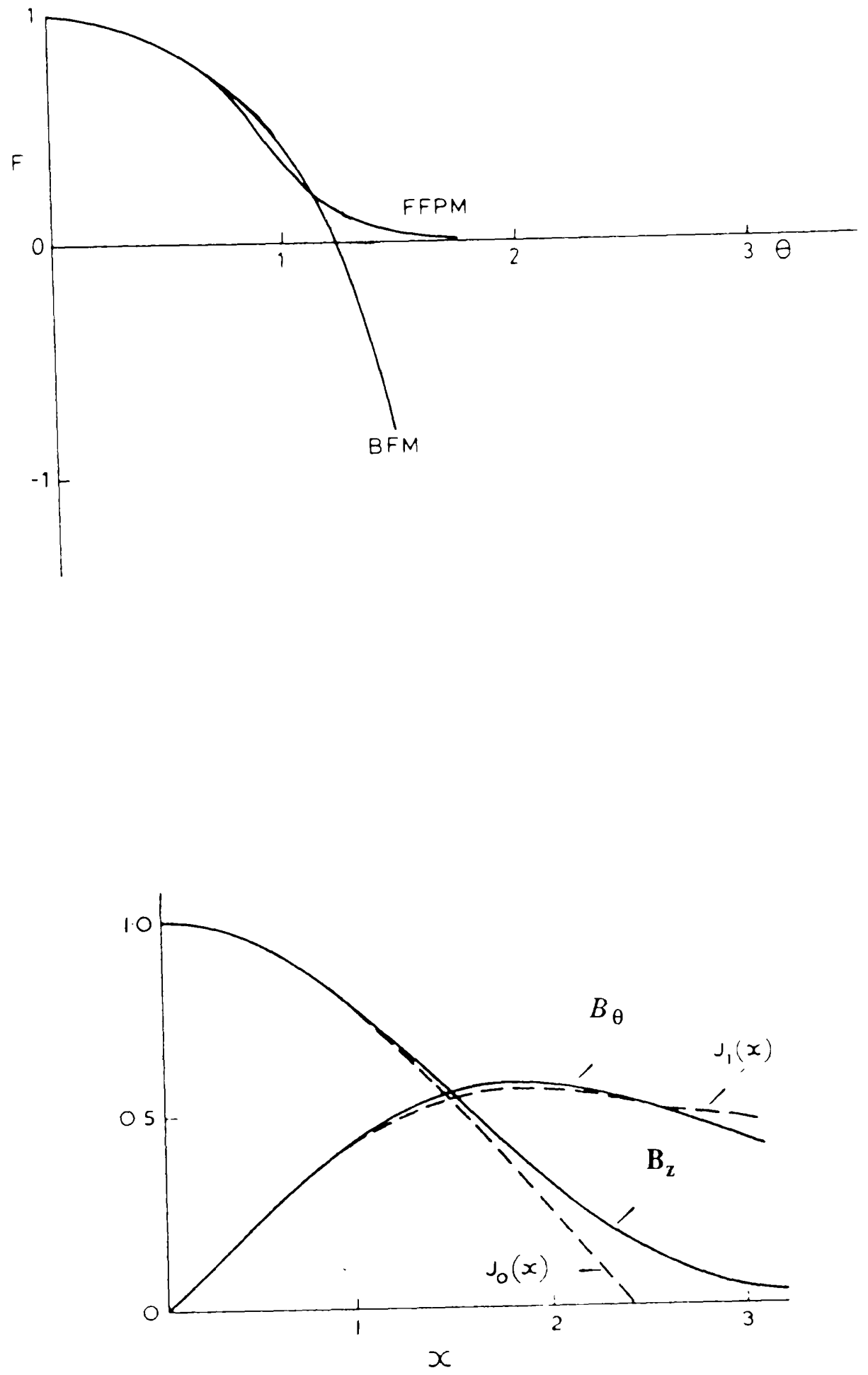

Figure 5.16

$F$ versus $\theta$ profile predicted by the FFPM and the BFM together with typical profiles of $B_{z}$ and $B_{\theta}$ (from Bodin and Newton, 1980 and Whiteman, 1965 ). 


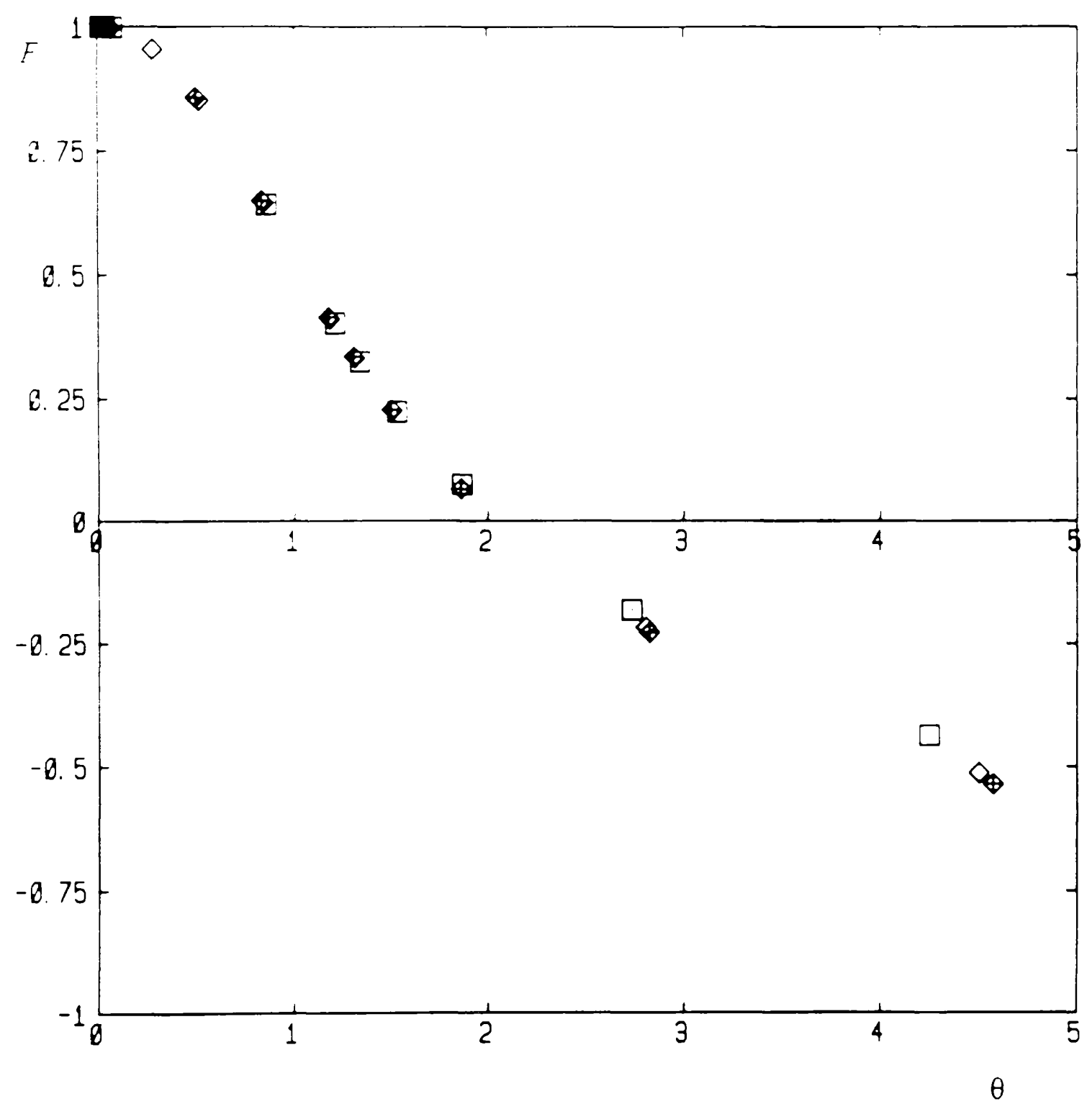

Figure 5.17

$F$ versus $\theta$ profiles for three different values of aspect-ratio ( 2.0, 4.0 and 10.0) shows an insignificant dependence on curvature. 


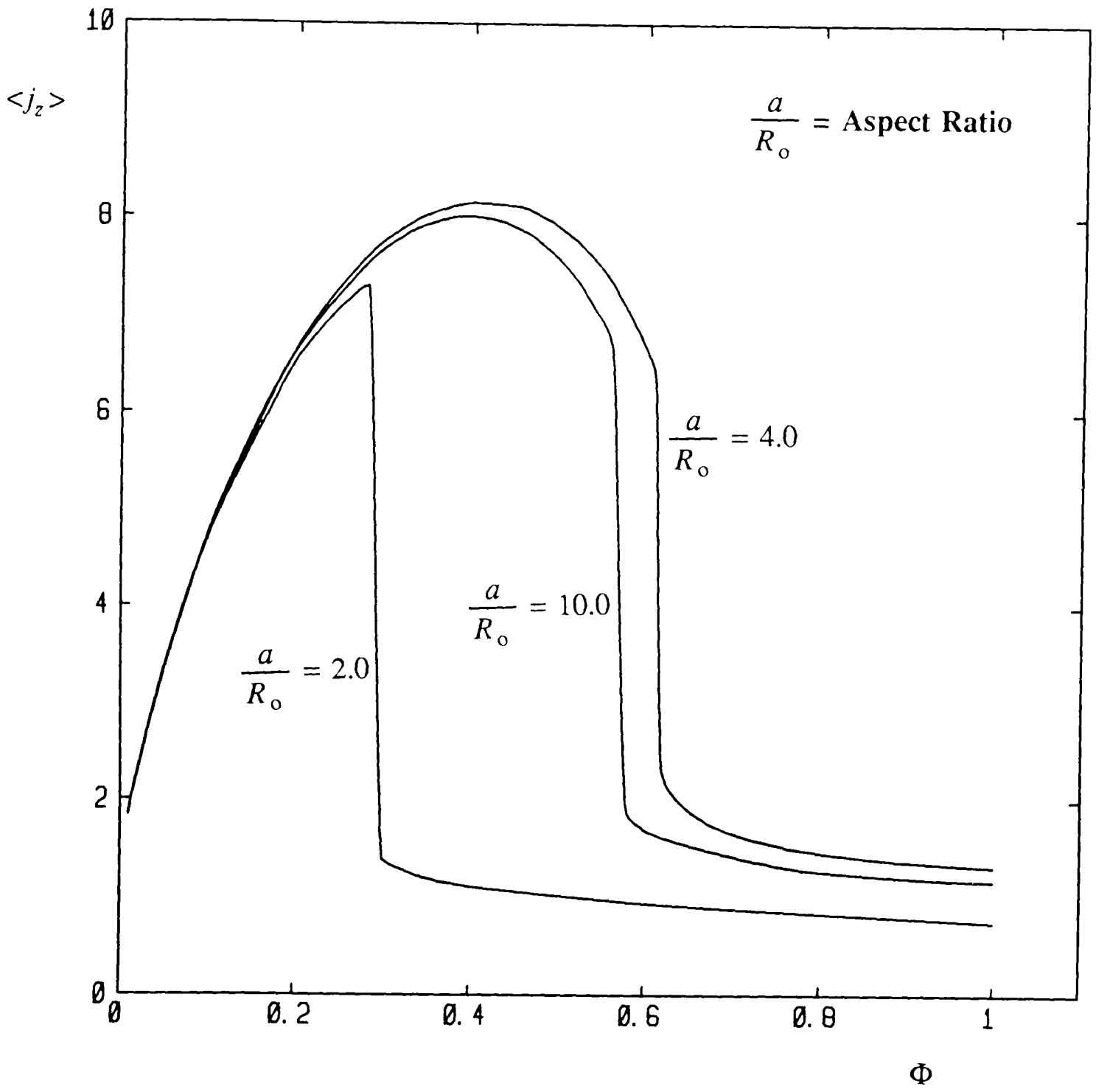

Figure 5.18

A plot of $\left\langle j_{z}\right\rangle$ versus $\Phi$ shows that the critical flux value is higher for aspect-ratio 4.0 than 2.0 but lies in between the two for aspect-ratio 10.0 . 


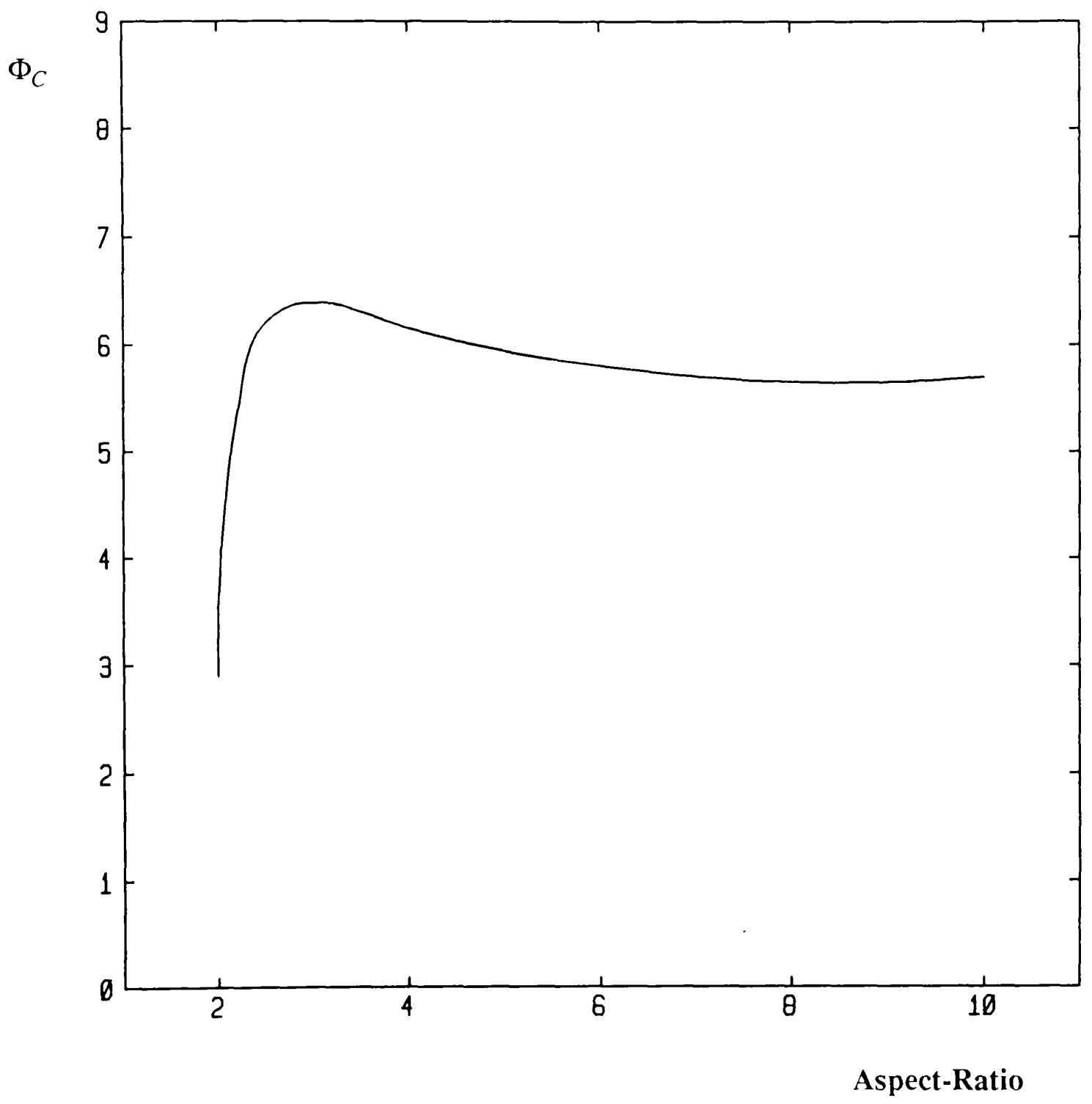

Figure 5.18b

A plot of critical axial flux $\Phi_{C}$ versus aspect-ratio shows that the critical flux increases, reaches a maximum and then decreases. The critical flux is seen to peek at aspect-ratio $\approx 3.0$. 

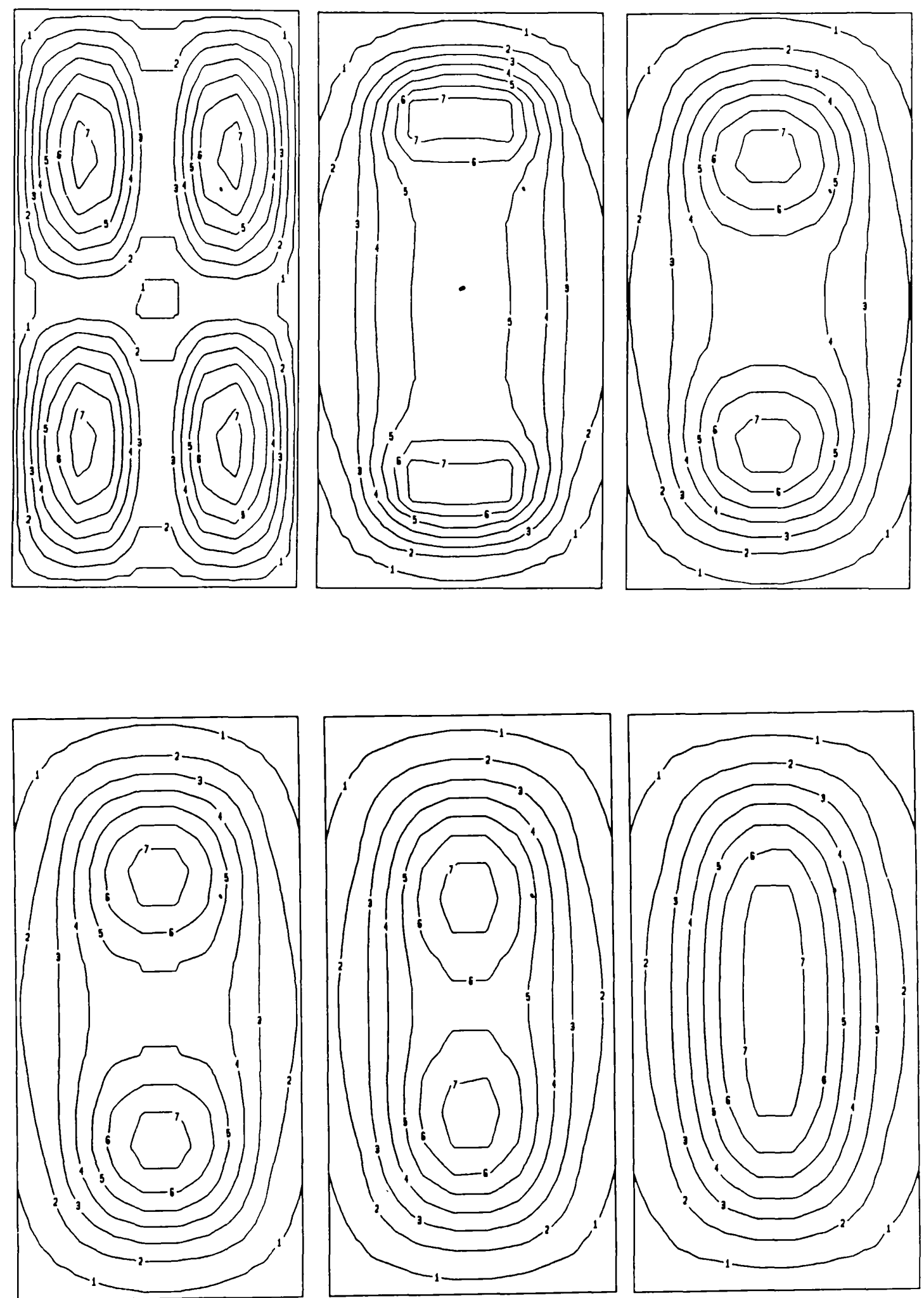

Figure 5.19

Time development of $B_{z}$ in a $1 \times 2$ rectangular cylinder. An initial configuration consisting of four helices evolves in such a way that the two nearest islands reconnect first to give just the two. These in turn reconnect to give just a single island as the final relaxed state. 

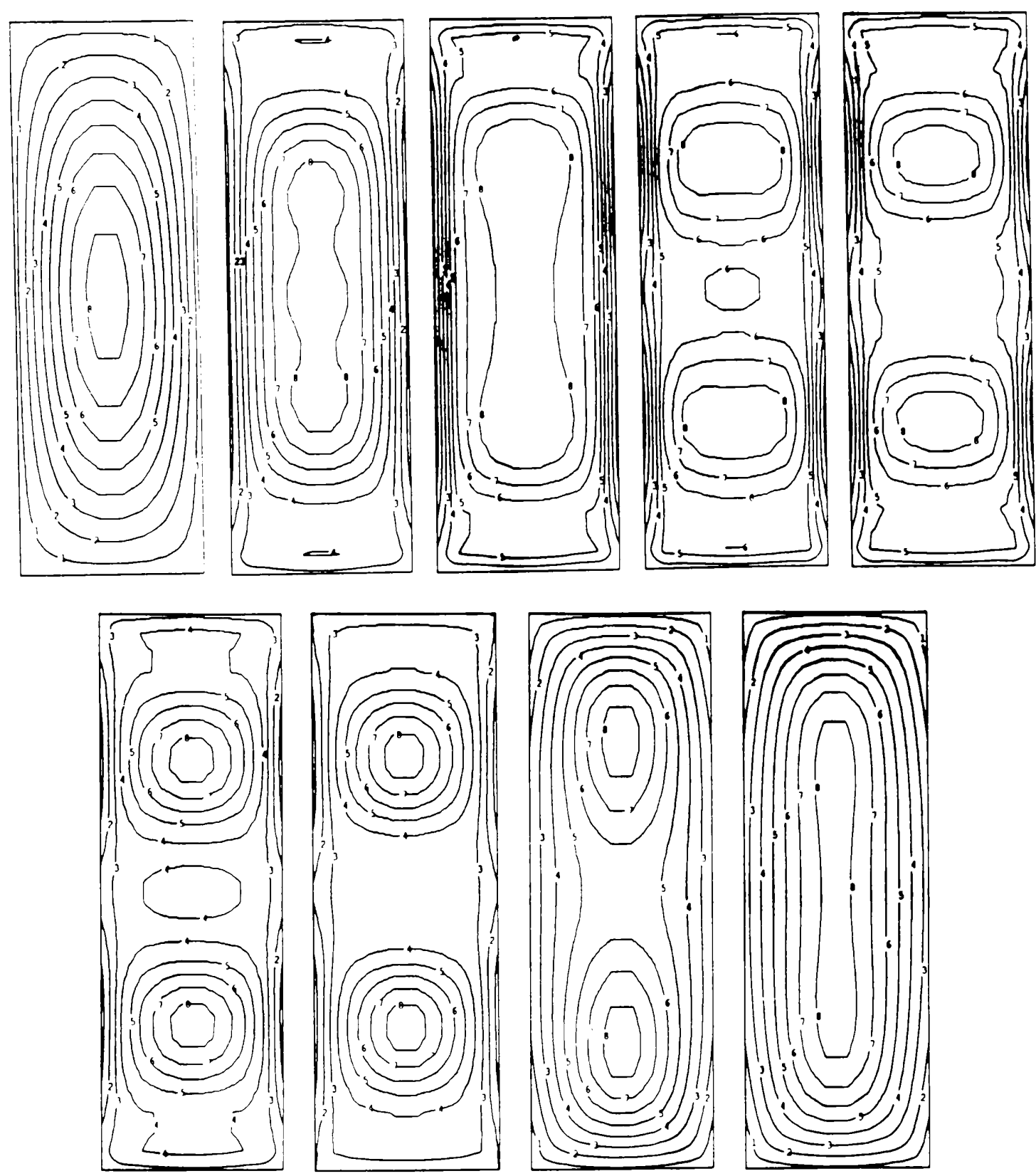

Figure 5.19b

The time development of $B_{z}$ in a $1 \times 3$ rectangular cylinder. The driving field is applied opposite to that already existing in the plasma. As the field in the plasma changes direction a series of reconnections follow. The initial single island reconnects to form multiple islands which subsequently go on to form just the single island as the relaxed state. 
A test run showing the evolution of the magnetic field lines in a $1 \times 1 \times 2$ rectangular cylinder. A helical type structure whose handedness is opposite to that of the relaxed state is chosen as the initial configuration. The evolution shows how the code is able to handle the complex evolution which takes place. The final state can seen to be an axisymmetric profile. 


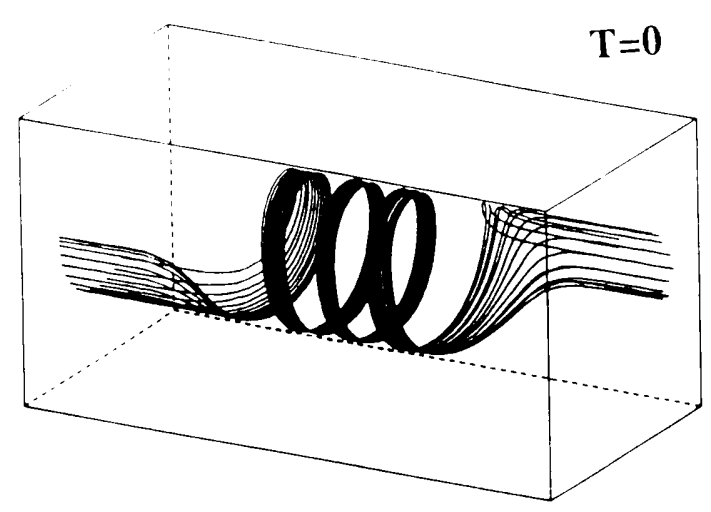

$\mathrm{T}=10$
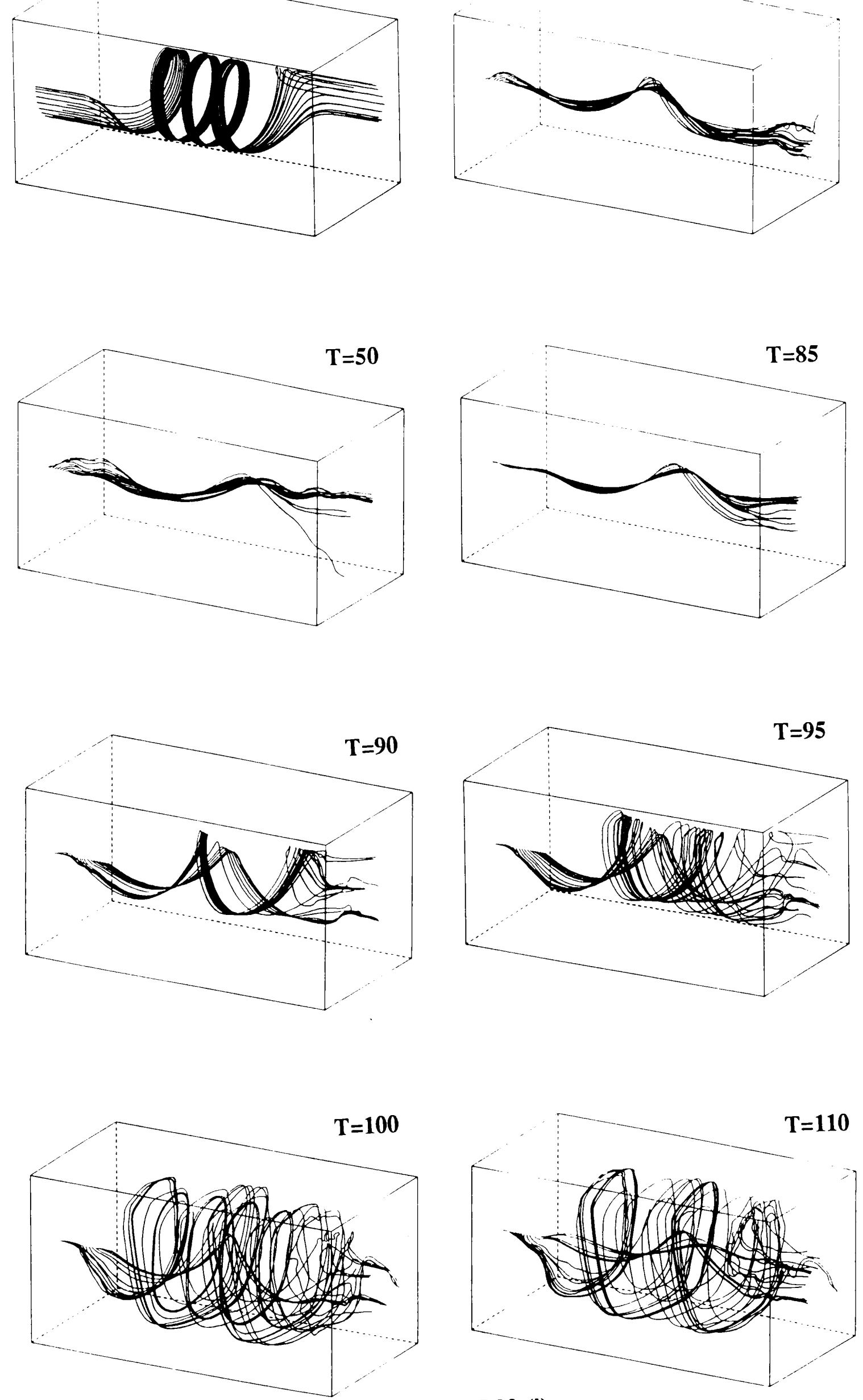

Figure 5.20 (i) 

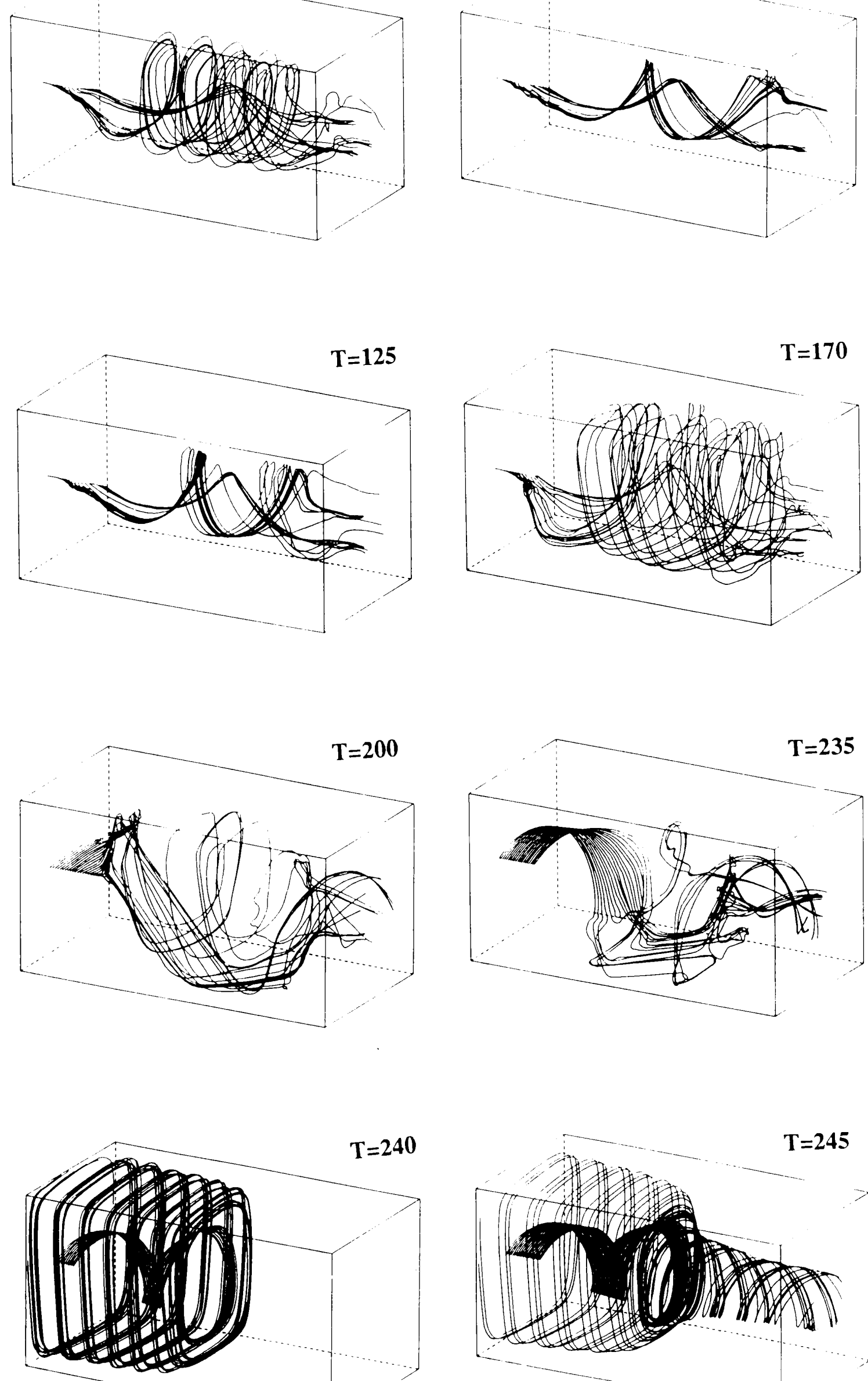

$\mathrm{T}=240$

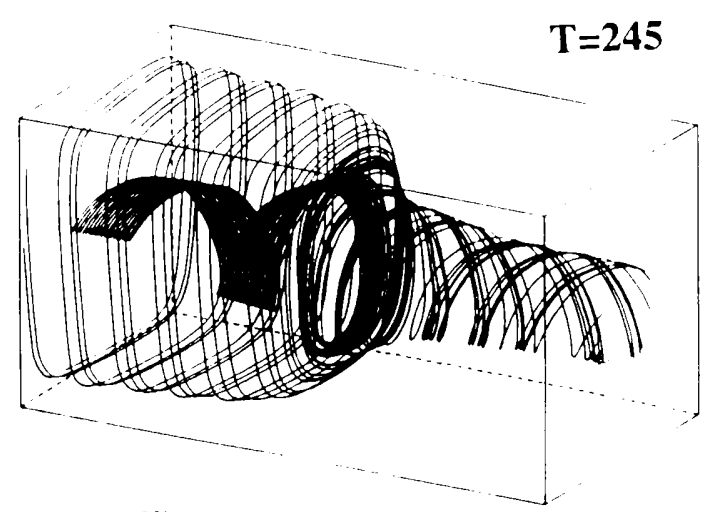

Figure 5.20 (ii) 


$$
m m
$$




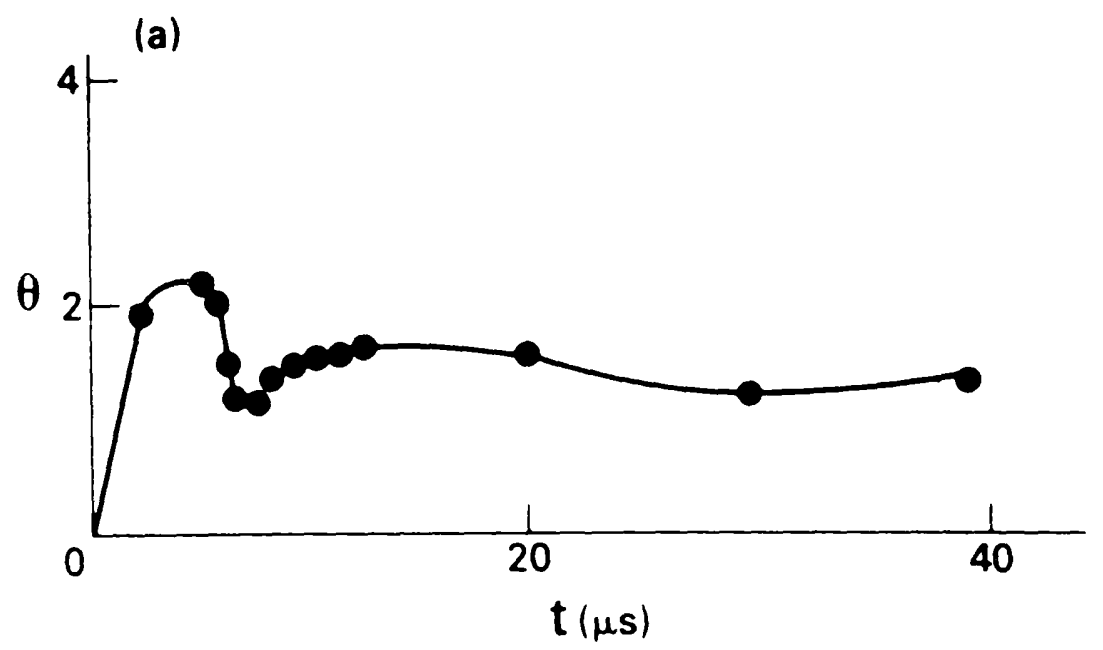

(b)

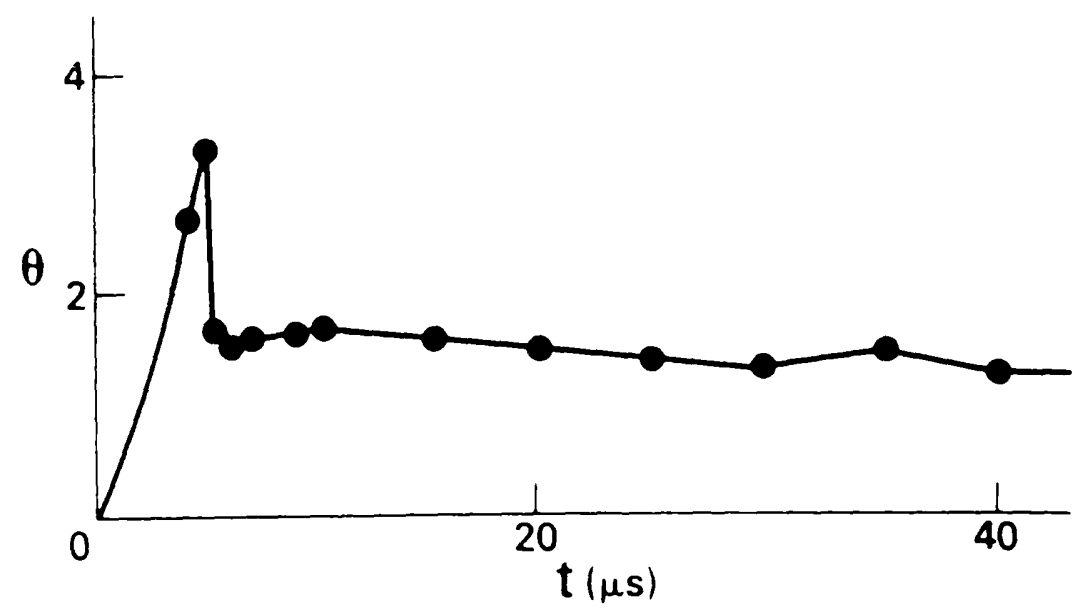

Figure 5.21

Plots showing limitation on $\theta$ (from Bodin and Newton, 1980 ). 
Equilibrium profiles fo increasing values of driving field to see the possibility of helical deformation. As can be seen, the toroidal current increases indefinitely with no sign of deformation. 

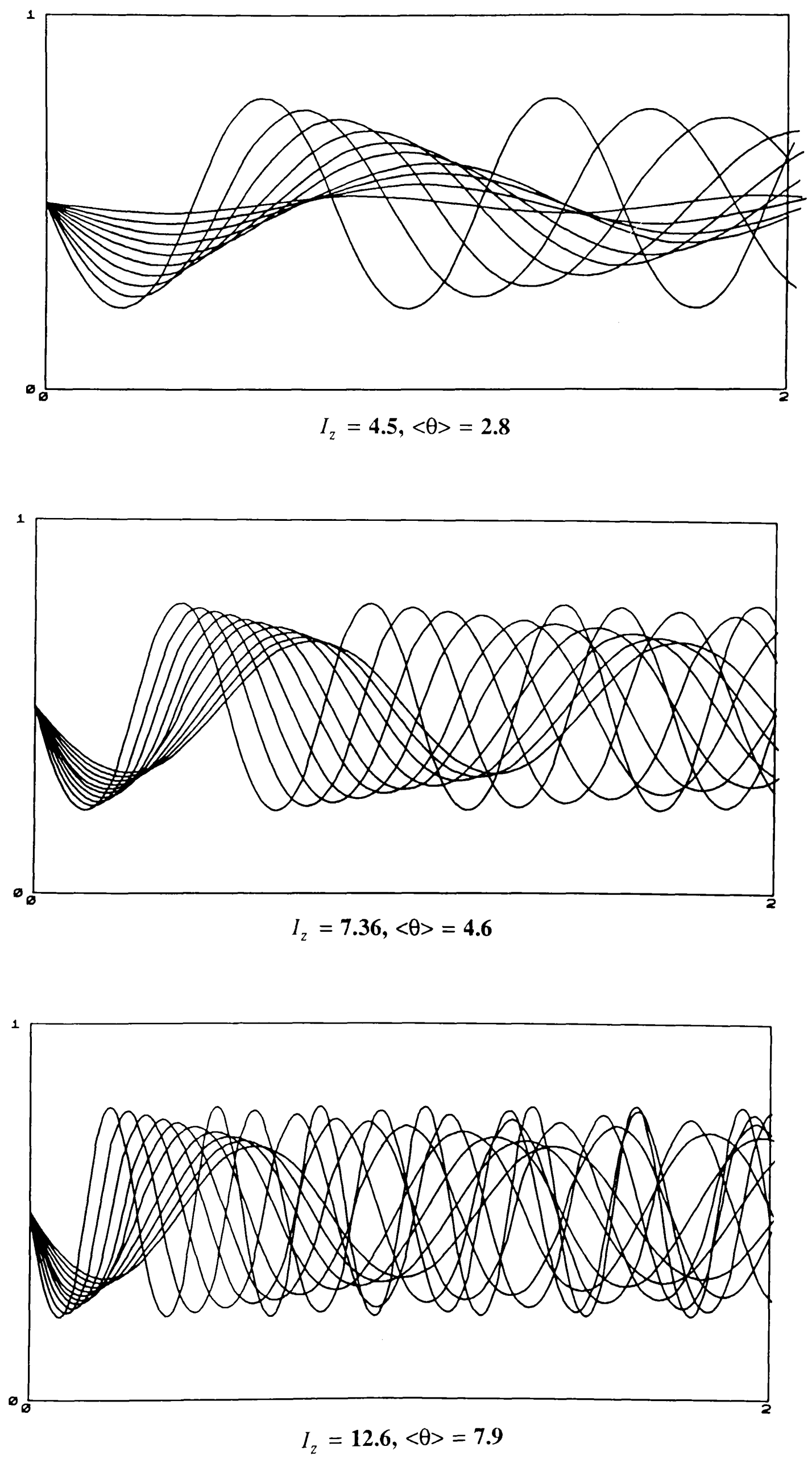

Figure 5.22 (i) 

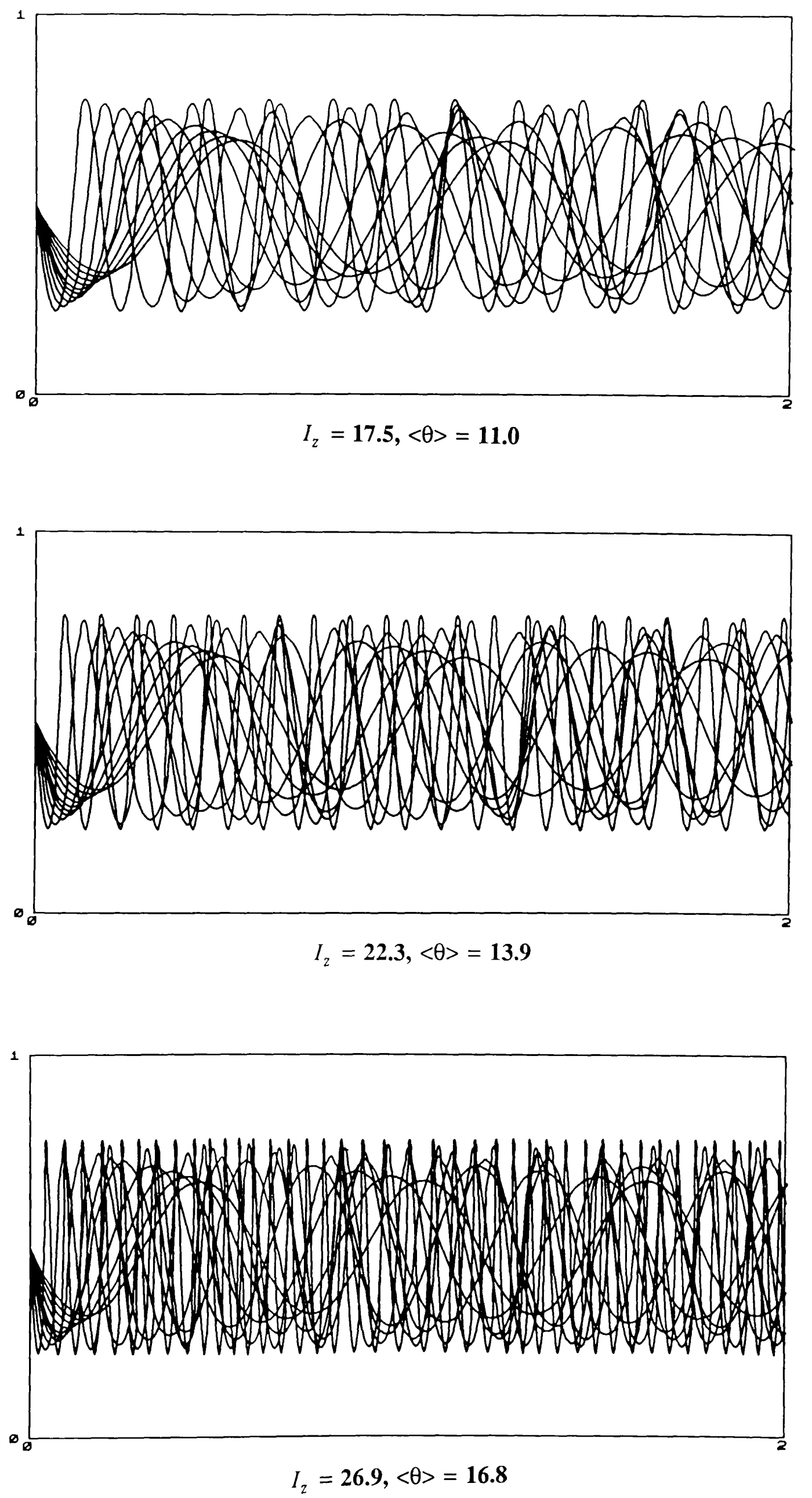

Figure 5.22 (ii) 


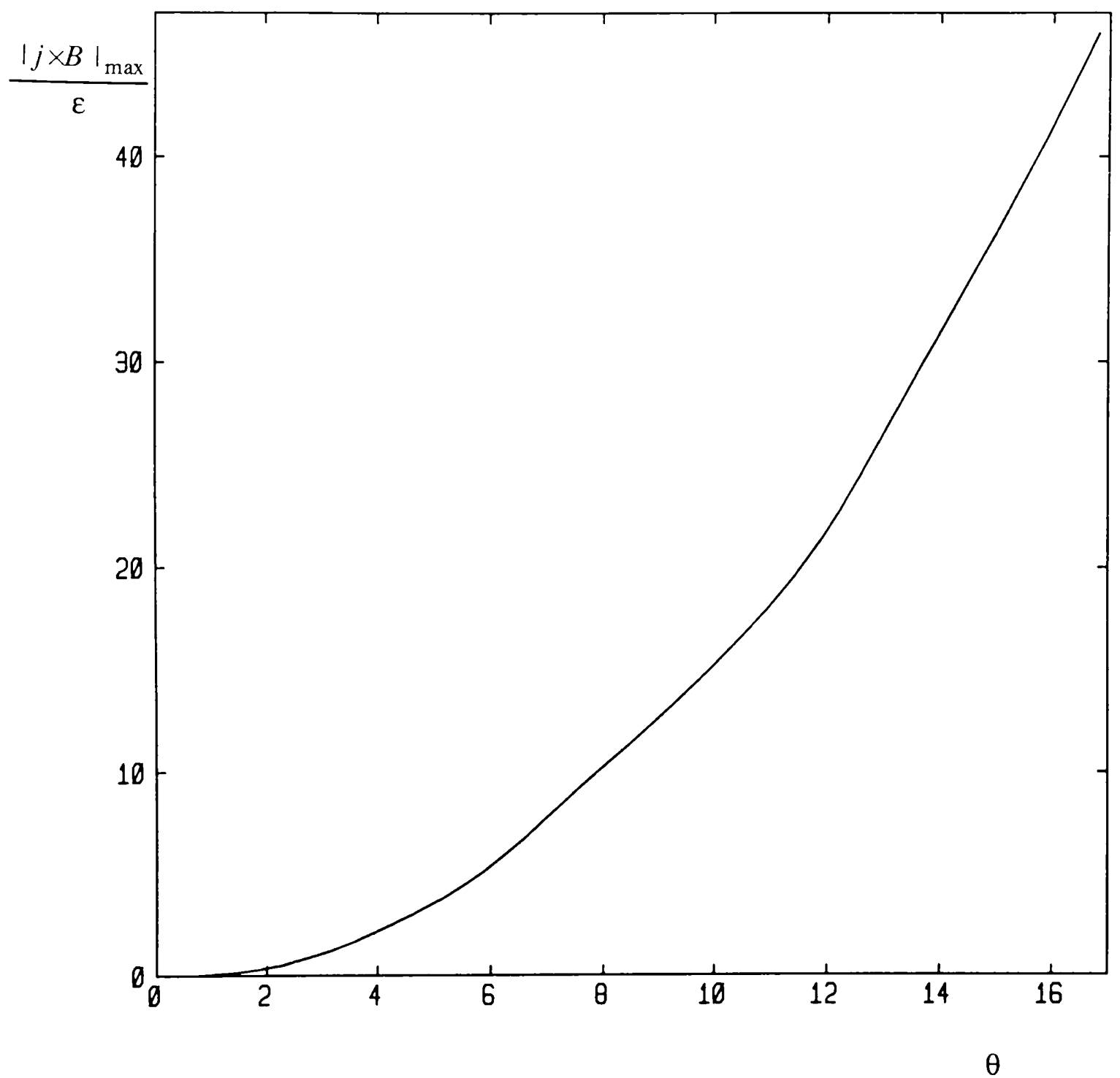

Figure 5.23

Plot shows how the force-free nature of the field is violated for large values of driving field. A higher resolution is thus required. 


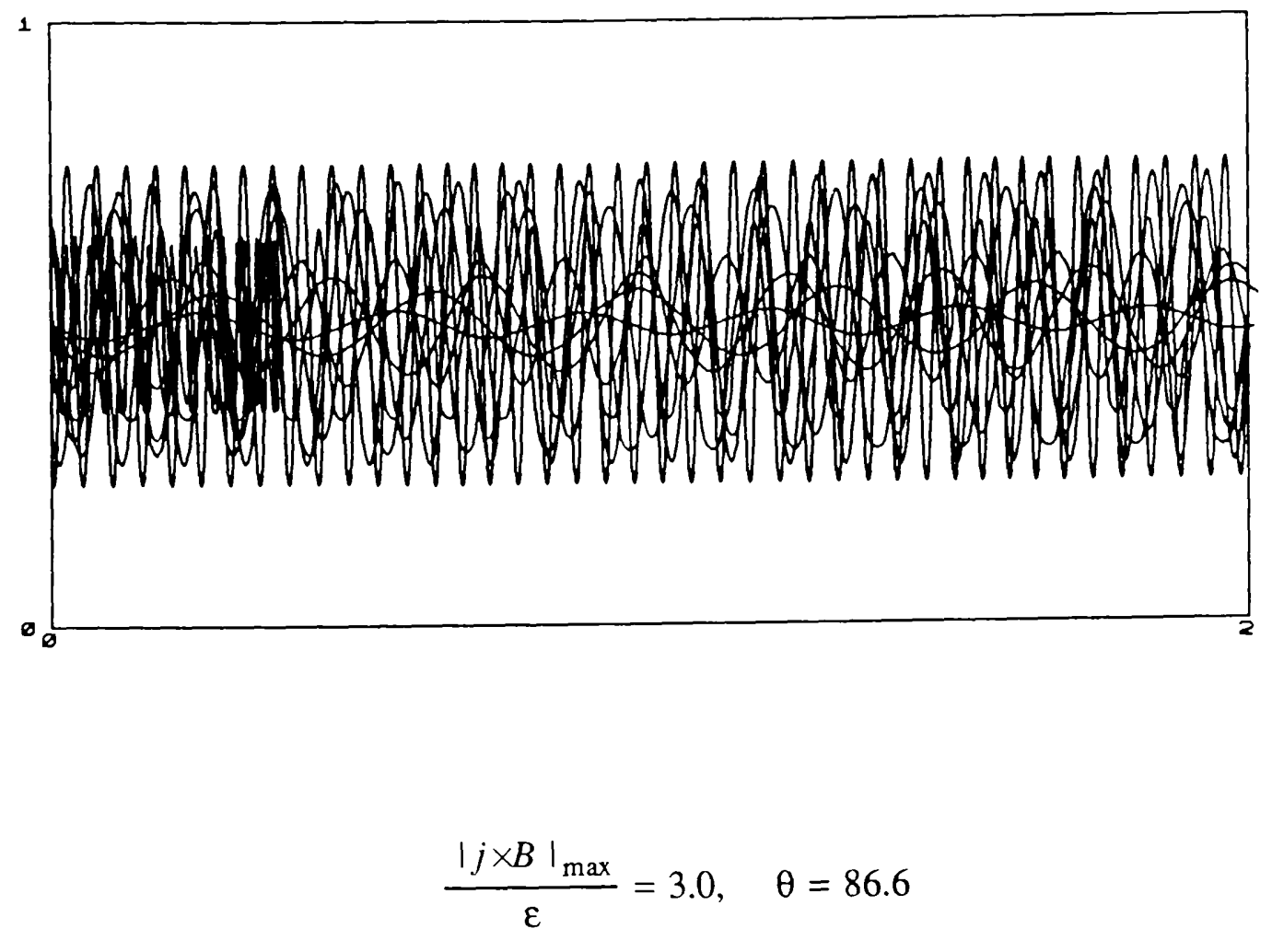

Figure 5.24

A high resolution result shows no sign of deformation. 
Magnetic field line structure for increasing values of driving field. Although the driving field has been given an axial dependence there is no sign of helical deformation. 

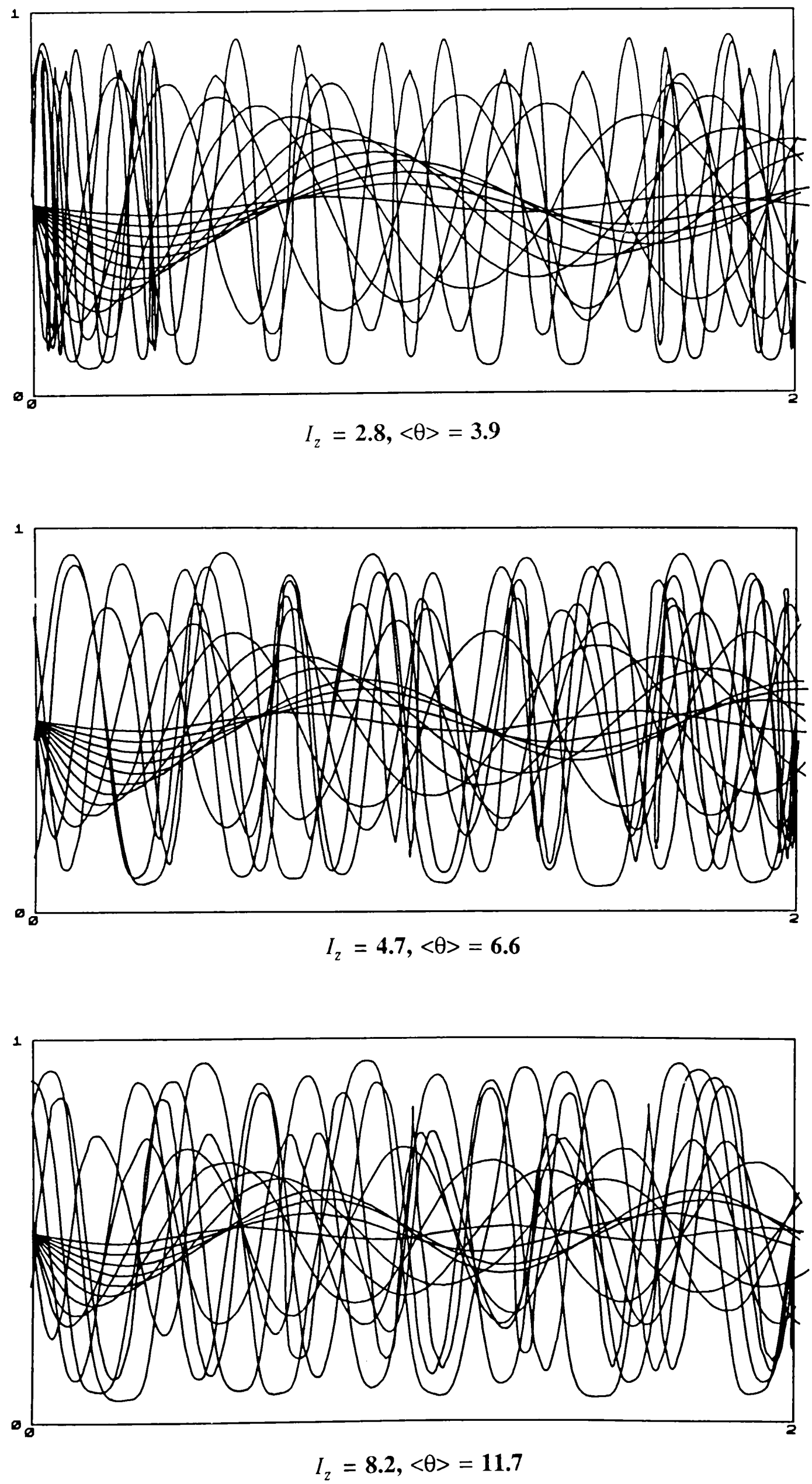

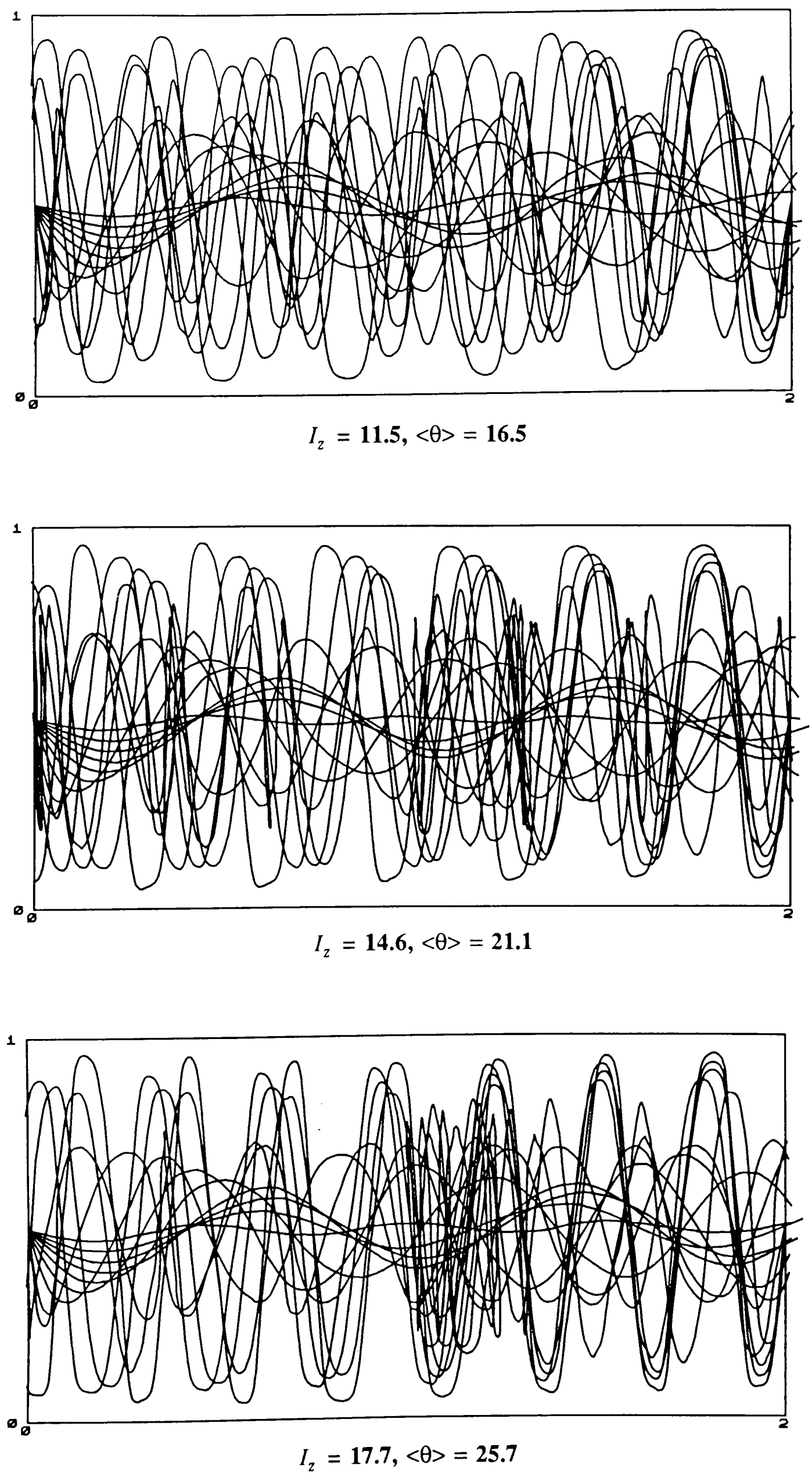
Magnetic field lines for the case where the axial flux has been given an axial dependence in order to generate non-axisymmetric relaxed states. Again there is no sign of helical deformation. 

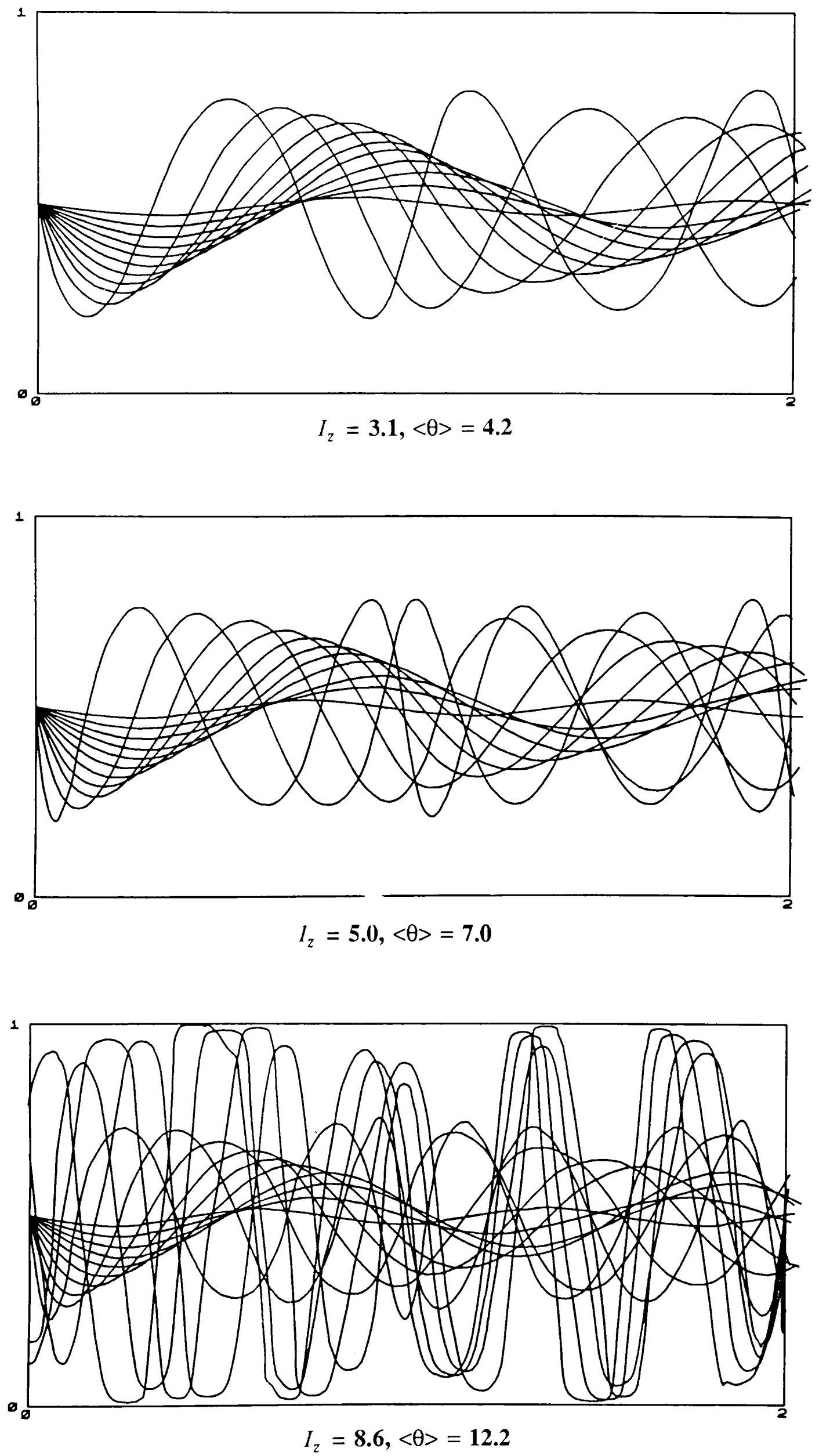

Figure 5.26 (i) 

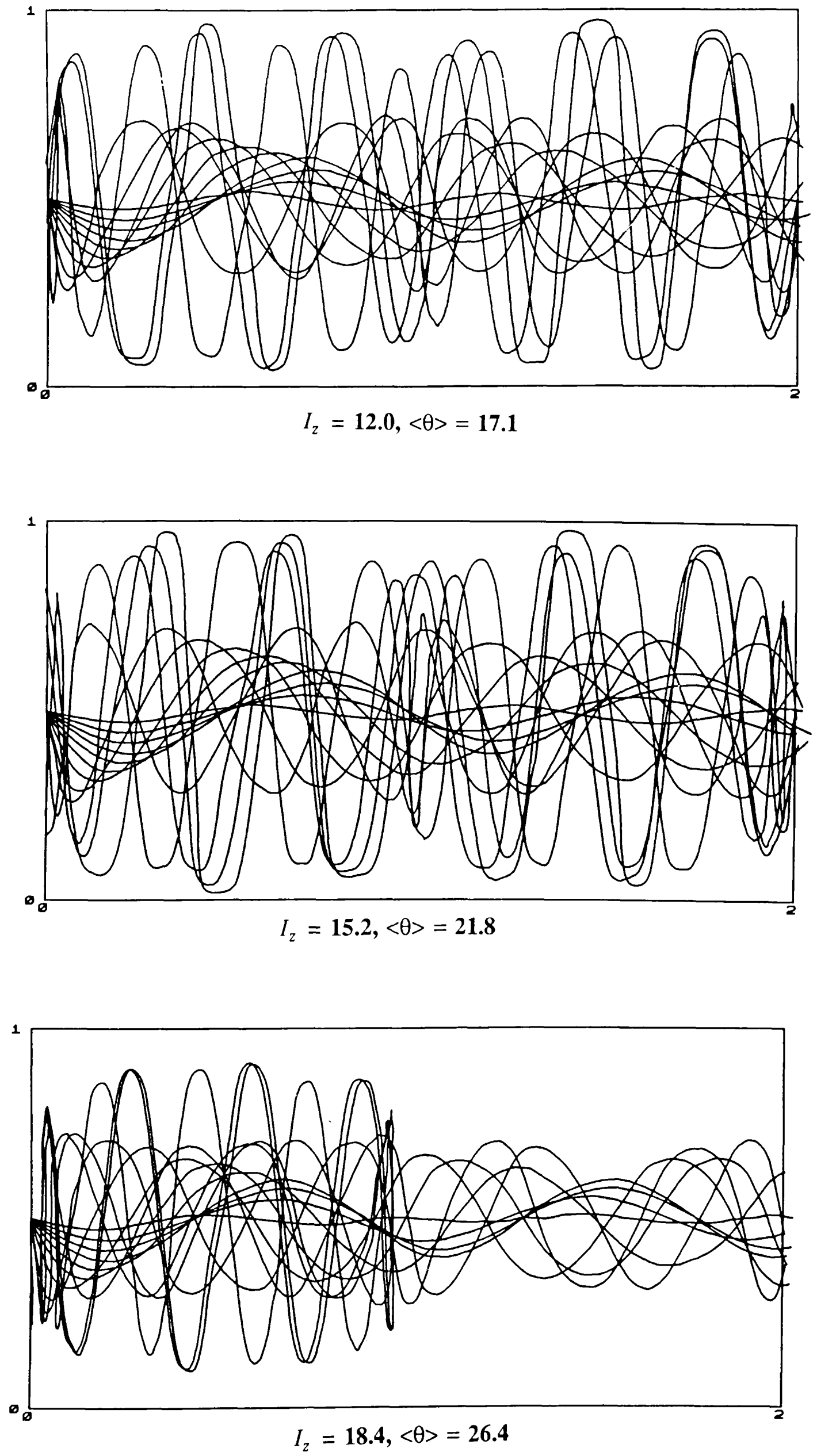

Figure 5.26 (ii) 


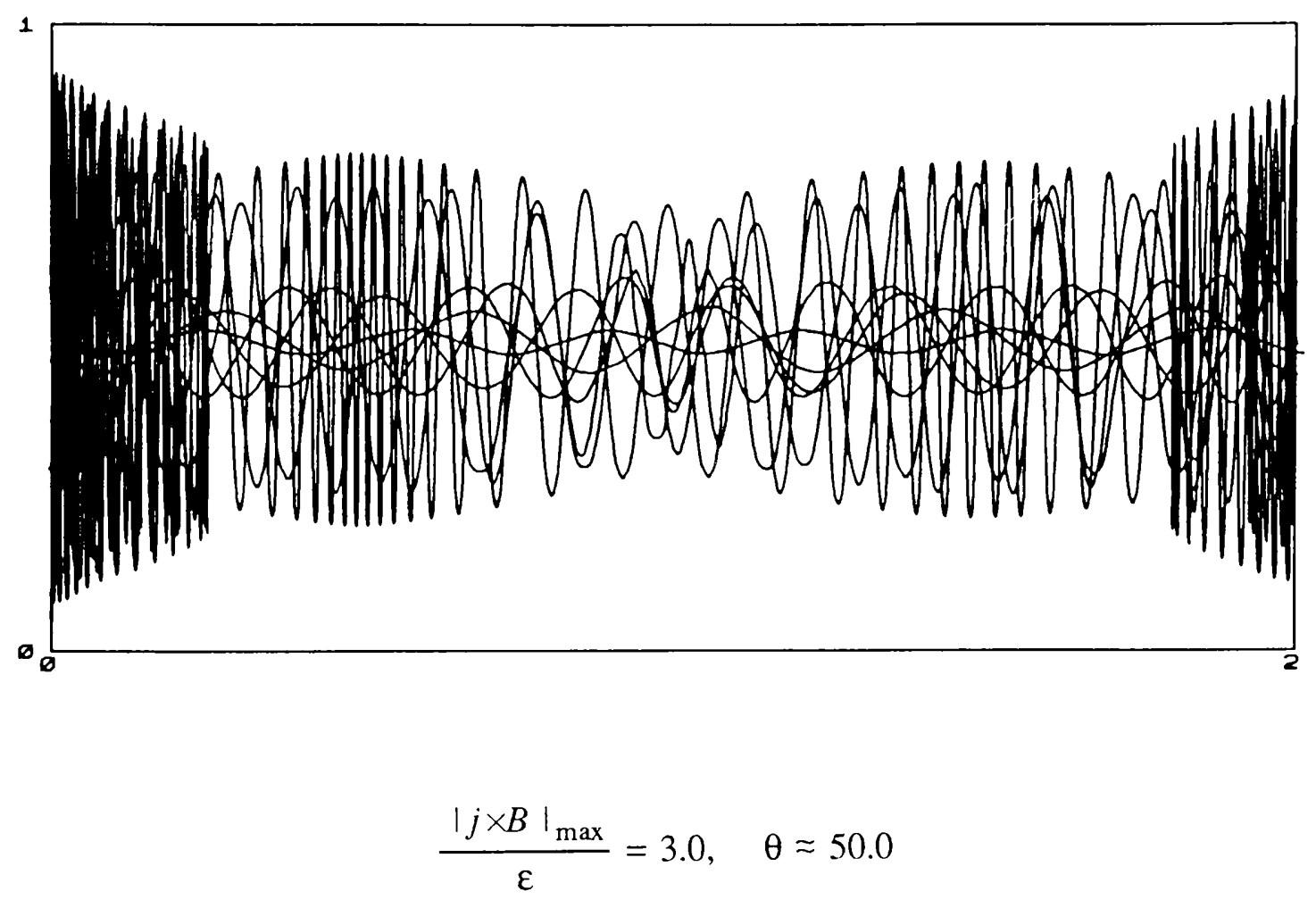

Figure 5.27

Magnetic field lines for a high resolution case where the axial flux has an axial dependence. Again there is no helical deformation.

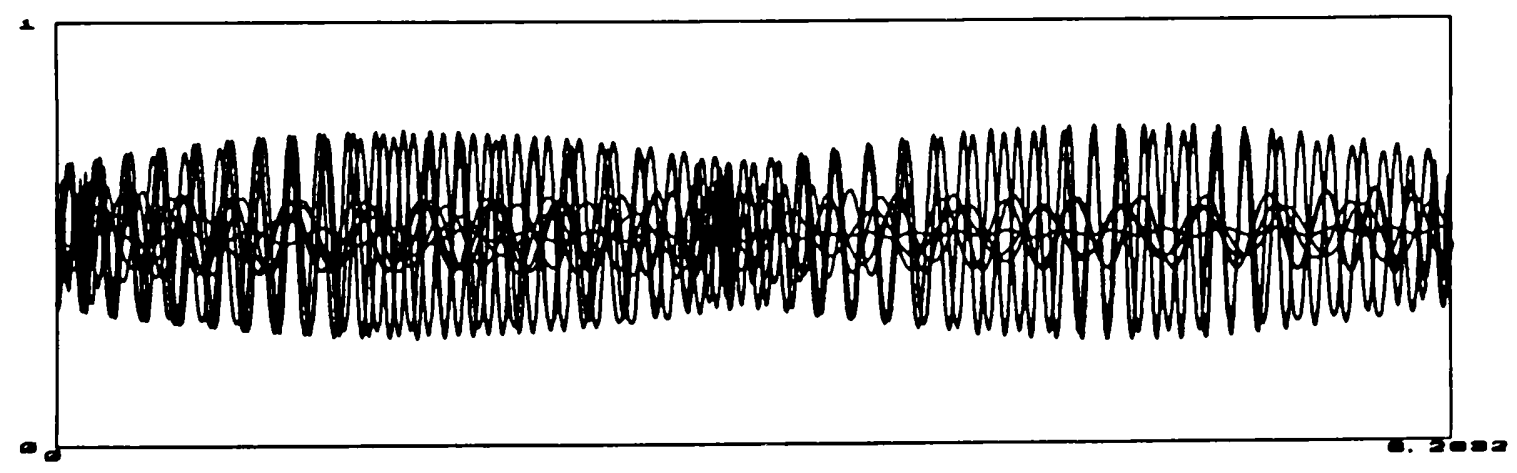

$$
\frac{|j \times B|_{\max }}{\varepsilon}=3.0, \quad \theta \approx 50.0
$$

Figure 5.28

High resolution result shows no helical deformation if the axial length of the cylinder is increased. 


\section{Appendix A}

\section{Evolution equations in terms of curvilinear co-ordinates}

The evolution equation

$$
\frac{\partial \mathbf{B}}{\partial t}=-\nabla \times \mathbf{E}
$$

can be written in component form, using curvilinear co-ordinates, as :

$$
\begin{aligned}
& \frac{\partial B_{1}}{\partial t}=\frac{1}{h_{2} h_{3}}\left\{\frac{\partial}{\partial u_{3}}\left(h_{2} E_{2}\right)-\frac{\partial}{\partial u_{2}}\left(h_{3} E_{3}\right)\right\} \\
& \frac{\partial B_{2}}{\partial t}=\frac{1}{h_{1} h_{3}}\left\{\frac{\partial}{\partial u_{1}}\left(h_{3} E_{3}\right)-\frac{\partial}{\partial u_{3}}\left(h_{1} E_{1}\right)\right\} \\
& \frac{\partial B_{3}}{\partial t}=\frac{1}{h_{1} h_{2}}\left\{\frac{\partial}{\partial u_{2}}\left(h_{1} E_{1}\right)-\frac{\partial}{\partial u_{1}}\left(h_{2} E_{2}\right)\right\}
\end{aligned}
$$

Ohm's Law, $\mathbf{E}=a F \mathbf{B}+b \nabla \times \mathbf{B}$, can be written as :

$$
\begin{aligned}
& E_{1}=a F B_{1}+\frac{b}{h_{2} h_{3}}\left\{\frac{\partial}{\partial u_{3}}\left(h_{2} B_{2}\right)-\frac{\partial}{\partial u_{2}}\left(h_{3} B_{3}\right)\right\} \\
& E_{2}=a F B_{2}+\frac{b}{h_{1} h_{3}}\left\{\frac{\partial}{\partial u_{1}}\left(h_{3} B_{3}\right)-\frac{\partial}{\partial u_{3}}\left(h_{1} B_{1}\right)\right\} \\
& E_{3}=a F B_{3}+\frac{b}{h_{1} h_{2}}\left\{\frac{\partial}{\partial u_{2}}\left(h_{1} B_{1}\right)-\frac{\partial}{\partial u_{1}}\left(h_{2} B_{2}\right)\right\}
\end{aligned}
$$


- 84 -

Substituting into the evolution equations and rearranging leads to :

$$
\begin{aligned}
h_{2} h_{3} \frac{\partial B_{1}}{\partial t} & =\frac{\partial}{\partial u_{2}}\left[\frac{h_{3}}{h_{1} h_{2}} \frac{\partial}{\partial u_{2}}\left(h_{1} B_{1}\right)\right]-\frac{\partial}{\partial u_{2}}\left[\frac{h_{3}}{h_{1} h_{2}} \frac{\partial}{\partial u_{1}}\left(h_{2} B_{2}\right)\right] \\
& +\frac{\partial}{\partial u_{3}}\left[\frac{h_{2}}{h_{1} h_{3}} \frac{\partial}{\partial u_{3}}\left(h_{1} B\right)\right]-\frac{\partial}{\partial u_{3}}\left(\frac{h_{2}}{h_{1} h_{3}} \frac{\partial}{\partial u_{1}}\left(h_{3} B_{3}\right)\right] \\
& -(1-\varepsilon)\left\{\frac{\partial}{\partial u_{2}}\left(h_{3} F B_{3}\right)-\frac{\partial}{\partial u_{3}}\left(h_{2} F B_{2}\right)\right\}
\end{aligned}
$$

$$
\begin{aligned}
h_{1} h_{3} \frac{\partial B_{2}}{\partial t} & =\frac{\partial}{\partial u_{3}}\left[\frac{h_{1}}{h_{2} h_{3}} \frac{\partial}{\partial u_{3}}\left(h_{2} B_{2}\right)\right]-\frac{\partial}{\partial u_{3}}\left[\frac{h_{1}}{h_{2} h_{3}} \frac{\partial}{\partial u_{2}}\left(h_{3} B_{3}\right)\right] \\
& +\frac{\partial}{\partial u_{1}}\left[\frac{h_{3}}{h_{1} h_{2}} \frac{\partial}{\partial u_{1}}\left(h_{2} B_{2}\right)\right]-\frac{\partial}{\partial u_{1}}\left(\frac{h_{3}}{h_{1} h_{2}} \frac{\partial}{\partial u_{2}}\left(h_{1} B_{1}\right)\right] \\
& -(1-\varepsilon)\left\{\frac{\partial}{\partial u_{3}}\left(h_{1} F B_{1}\right)-\frac{\partial}{\partial u_{1}}\left(h_{3} F B_{3}\right)\right\}
\end{aligned}
$$

$$
\begin{aligned}
h_{1} h_{2} \frac{\partial B_{3}}{\partial t} & =\frac{\partial}{\partial u_{1}}\left[\frac{h_{2}}{h_{1} h_{3}} \frac{\partial}{\partial u_{1}}\left(h_{3} B_{3}\right)\right]-\frac{\partial}{\partial u_{1}}\left(\frac{h_{2}}{h_{1} h_{3}} \frac{\partial}{\partial u_{3}}\left(h_{1} B_{1}\right)\right] \\
& +\frac{\partial}{\partial u_{2}}\left[\frac{h_{1}}{h_{2} h_{3}} \frac{\partial}{\partial u_{2}}\left(h_{3} B_{3}\right)\right]-\frac{\partial}{\partial u_{2}}\left(\frac{h_{1}}{h_{2} h_{3}} \frac{\partial}{\partial u_{3}}\left(h_{2} B_{2}\right)\right] \\
& -(1-\varepsilon)\left\{\frac{\partial}{\partial u_{1}}\left(h_{2} F B_{2}\right)-\frac{\partial}{\partial u_{2}}\left(h_{1} F B_{1}\right)\right\}
\end{aligned}
$$




\section{Cartesian co-ordinates}

setting $u 1=x, u 2=y, u 3=z$, and $h 1=h 2=h 3=1$ gives :

$$
\begin{aligned}
\frac{\partial B_{x}}{\partial t} & =\frac{\partial}{\partial y}\left[\frac{\partial B_{x}}{\partial y}\right]+\frac{\partial}{\partial z}\left[\frac{\partial B_{x}}{\partial z}\right]-\frac{\partial^{2} B_{y}}{\partial x \partial y}-\frac{\partial^{2} B_{z}}{\partial x \partial z} \\
& -(1-\varepsilon)\left\{\frac{\partial}{\partial y}\left(F B_{z}\right)-\frac{\partial}{\partial z}\left(F B_{y}\right)\right\} \\
\frac{\partial B_{y}}{\partial t}= & \frac{\partial}{\partial x}\left[\frac{\partial B_{y}}{\partial x}\right]+\frac{\partial}{\partial z}\left[\frac{\partial B_{y}}{\partial z}\right]-\frac{\partial^{2} B_{x}}{\partial x \partial y}-\frac{\partial^{2} B_{z}}{\partial y \partial z} \\
& -(1-\varepsilon)\left\{\frac{\partial}{\partial z}\left(F B_{x}\right)-\frac{\partial}{\partial x}\left(F B_{z}\right)\right\} \\
\frac{\partial B_{z}}{\partial t}= & \frac{\partial}{\partial x}\left[\frac{\partial B_{z}}{\partial x}\right]+\frac{\partial}{\partial y}\left[\frac{\partial B_{z}}{\partial y}\right]-\frac{\partial^{2} B_{x}}{\partial x \partial z}-\frac{\partial^{2} B_{y}}{\partial y \partial z} \\
& -(1-\varepsilon)\left\{\frac{\partial}{\partial x}\left(F B_{y}\right)-\frac{\partial}{\partial y}\left(F B_{x}\right)\right\}
\end{aligned}
$$

\section{Toroidal co-ordinates}

setting $u 1=x, u 2=y, u 3=\phi, h 1=h 2=1$ and $h 3=R$ gives :

$$
\begin{aligned}
\frac{\partial B_{x}}{\partial t} & =\frac{\partial}{\partial y}\left[\frac{\partial B_{x}}{\partial y}\right]-\frac{\partial^{2} B_{y}}{\partial y \partial x}+\frac{1}{R^{2}} \frac{\partial}{\partial \phi}\left[\frac{\partial B_{x}}{\partial \phi}\right]-\frac{1}{R^{2}} \frac{\partial^{2}\left(R B_{0}\right)}{\partial \phi \partial x} \\
& -(1-\varepsilon)\left\{\frac{\partial}{\partial y}\left(F B_{\phi}\right)-\frac{\partial}{\partial \phi}\left(F B_{y}\right)\right\}
\end{aligned}
$$




$$
\begin{aligned}
& \frac{\partial B_{y}}{\partial t}=\frac{1}{R^{2}} \frac{\partial}{\partial \phi}\left(\frac{\partial B_{y}}{\partial \phi}\right)-\frac{1}{R} \frac{\partial^{2} B_{\phi}}{\partial \phi \partial y}+\frac{1}{R} \frac{\partial}{\partial x}\left[R \frac{\partial B_{y}}{\partial x}\right)-\frac{1}{R} \frac{\partial}{\partial x}\left[R \frac{\partial B_{x}}{\partial y}\right) \\
& -(1-\varepsilon)\left\{\frac{1}{R} \frac{\partial}{\partial \phi}\left(F B_{x}\right)-\frac{1}{R} \frac{\partial}{\partial x}\left(R F B_{\phi}\right)\right\} \\
& \frac{\partial B_{\circ}}{\partial t}=\frac{\partial}{\partial x}\left[\frac{1}{R} \frac{\partial}{\partial x}\left(R B_{\phi}\right)\right]-\frac{\partial}{\partial x}\left[\frac{1}{R} \frac{\partial B_{x}}{\partial \phi}\right]+\frac{\partial}{\partial y}\left[\frac{\partial B_{\circ}}{\partial y}\right)-\frac{1}{R} \frac{\partial^{2} B_{y}}{\partial y \partial \phi} \\
& -(1-\varepsilon)\left\{\frac{\partial}{\partial x}\left(F B_{y}\right)-\frac{\partial}{\partial y}\left(F B_{x}\right)\right\}
\end{aligned}
$$

\section{Alternative representation of evolution equations}

The mixed derivatives arising in the above evolution equations can be eliminated using $\nabla \cdot \mathbf{B}=0$.

\section{Cartesians}

$$
\begin{aligned}
& \frac{\partial B_{x}}{\partial t}=\nabla^{2} B_{x}-(1-\varepsilon)\left\{B_{z} \frac{\partial F}{\partial y}+F \frac{\partial B_{z}}{\partial y}-B_{y} \frac{\partial F}{\partial z}-F \frac{\partial B_{y}}{\partial z}\right\} \\
& \frac{\partial B_{y}}{\partial t}=\nabla^{2} B_{y}-(1-\varepsilon)\left\{B_{x} \frac{\partial F}{\partial z}+F \frac{\partial B_{x}}{\partial x}-B_{z} \frac{\partial F}{\partial x}-F \frac{\partial B_{z}}{\partial x}\right\} \\
& \frac{\partial B_{z}}{\partial t}=\nabla^{2} B_{z}-(1-\varepsilon)\left\{B_{y} \frac{\partial F}{\partial x}+F \frac{\partial B_{y}}{\partial x}-B_{x} \frac{\partial F}{\partial y}-F \frac{\partial B_{x}}{\partial y}\right\}
\end{aligned}
$$




\section{Toroidal co-ordinates}

$$
\begin{aligned}
\frac{\partial B_{x}}{\partial t}= & \frac{\partial^{2} B_{x}}{\partial x^{2}}+\frac{\partial^{2} B_{x}}{\partial y^{2}}+\frac{1}{R^{2}} \frac{\partial^{2} B_{x}}{\partial \phi^{2}}+\frac{1}{R} \frac{\partial B_{x}}{\partial x}-\frac{B_{x}}{R^{2}}-\frac{2}{R^{2}} \frac{\partial B_{\circ}}{\partial \phi} \\
& -(1-\varepsilon)\left\{B_{\circ} \frac{\partial F}{\partial y}+F \frac{\partial B_{\circ}}{\partial y}-\frac{B_{y}}{R} \frac{\partial F}{\partial \phi}+\frac{F}{R} \frac{\partial B_{y}}{\partial \phi}\right\} \\
\frac{\partial B_{y}}{\partial t}= & \frac{\partial^{2} B_{y}}{\partial x^{2}}+\frac{\partial^{2} B_{y}}{\partial y^{2}}+\frac{1}{R^{2}} \frac{\partial^{2} B_{y}}{\partial \phi^{2}}+\frac{1}{R} \frac{\partial B_{y}}{\partial x} \\
& -(1-\varepsilon)\left\{\frac{B_{x}}{R} \frac{\partial F}{\partial \phi}+\frac{F}{R} \frac{\partial B_{x}}{\partial \phi}-B_{\circ} \frac{\partial F}{\partial x}-F \frac{\partial B_{\circ}}{\partial x}-\frac{F B_{\circ}}{R}\right\} \\
\frac{\partial B_{\phi}}{\partial t}= & \frac{\partial^{2} B_{\phi}}{\partial x^{2}}+\frac{\partial^{2} B_{\diamond}}{\partial y^{2}}+\frac{1}{R^{2}} \frac{\partial^{2} B_{\phi}}{\partial \phi^{2}}+\frac{\partial}{\partial x}\left[\frac{B_{z}}{R}\right]+\frac{2}{R^{2}} \frac{\partial B_{x}}{\partial \phi} \\
& -(1-\varepsilon)\left\{B_{y} \frac{\partial F}{\partial x}+F \frac{\partial B_{y}}{\partial x}-B_{x} \frac{\partial F}{\partial y}-F \frac{\partial B_{x}}{\partial y}\right\}
\end{aligned}
$$

\section{Stream Function}

Define the stream function $\psi$ by :

$R B_{x}=\frac{\partial \psi}{\partial y}$

$R B_{y}=-\frac{\partial \psi}{\partial x}$ 
then in terms of $\psi$ the evolution equations can be written as :

$\frac{\partial \psi}{\partial t}=\nabla^{2} \psi-\frac{1}{R} \frac{\partial \psi}{\partial x}-(1-\varepsilon) R F B_{\phi}+\alpha R$

$\frac{\partial B_{\circ}}{\partial t}=\nabla^{2} B_{\phi}+\frac{\partial}{\partial x}\left[\frac{B_{\mathrm{o}}}{R}\right]+(1-\varepsilon)\left\{\frac{\partial}{\partial x}\left[\frac{F}{R} \frac{\partial \psi}{\partial x}\right)+\frac{\partial}{\partial y}\left(\frac{F}{R} \frac{\partial \psi}{\partial y}\right)\right\}$

where :

$F=\frac{R\left\{B_{\phi} \nabla^{2} \psi-\frac{\partial \psi}{\partial x} \frac{\partial B_{\phi}}{\partial x}-\frac{\partial \psi}{\partial y} \frac{\partial B_{\phi}}{\partial y}\right\}-2 B_{\phi} \frac{\partial \psi}{\partial x}}{(\nabla \psi)^{2}+\left(R B_{\phi}\right)^{2}}$ 


\section{Appendix B}

\section{Summation of difference terms}

The local axial flux has been defined as :

$\Delta \Phi=\frac{\Delta x \Delta y}{4}\left(B_{i, j}+B_{i+1, j}+B_{i, j+1}+B_{i+1, j+1}\right)$

The total axial flux is then :

$\Phi=\frac{\Delta x \Delta y}{4} \sum_{i=1}^{m-1} \sum_{j=1}^{n-1}\left(B_{i, j}+B_{i+1, j}+B_{i, j+1}+B_{i+1, j+1}\right)$

where $B$ is the axial component of the magnetic field. The number of mesh points along $x$ and $y$ is given by $m$ and $n$ respectively.

Now consider various derivatives.

1. $\frac{\partial \mathbf{B}}{\partial \mathbf{t}}=\frac{\partial \mathbf{B}}{\partial \mathbf{x}}$

In terms of differences approximations :

$B^{n+1}=B+\frac{\Delta t}{2 \Delta x}\left(B_{i+1, j}-B_{i-1, j}\right)$

and :

$\Delta \Phi^{n+1}=\Delta \Phi+\frac{\Delta t \Delta y}{8} \psi$

where : 
$\psi=\left(B_{i+1, j}-B_{i-1, j}+B_{i+2, j}-B_{i, j}+B_{i+1, j+1}-B_{i-1, j+1}+B_{i+2, j+1}-B_{i, j+1}\right)$

The total axial flux is then :

$\Phi^{n+1}=\Phi+\frac{\Delta t \Delta y}{8} \sum_{i=1}^{m-1} \sum_{j=1}^{n-1} \psi$

$=\Phi+\frac{\Delta t \Delta y}{8}\left\{\sum_{i=1}^{m-1}\left[2 \sum_{j=2}^{n-1} \psi+\psi_{j=1}+\psi_{j=n}\right]\right\}$

but :

$\sum_{i=1}^{m-2} \psi=$

$$
B_{0}+B_{1}-B_{2}-B_{3}
$$$$
+B_{1}+B_{2}-B_{3}-B_{4}
$$$$
+B_{2}+B_{3}-B_{4}-B_{5}
$$$$
+B_{3}+B_{4}-B_{5}-B_{6}
$$

$+B_{m-5}+B_{m-4}-B_{m-3}-B_{m-2}$

$+B_{m-4}+B_{m-3}-B_{m-2}-B_{m-1}$

$+B_{m-3}+B_{m-2}-B_{m-1}-B_{m}$ 
$+B_{m-2}+B_{m-1}-B_{m}-B_{m}$

$=\left(B_{0}+2 B_{1}+B_{2}\right)-\left(B_{m-1}+2 B_{m}+B_{m+1}\right)$

therefor:

$\Phi^{n+1}=\Phi+\frac{\Delta t \Delta y}{8}\left[2 \sum_{j=2}^{n-1} \Psi+\Psi_{j=1}+\Psi_{j=n}\right]$

where :

$=\left(B_{0}+2 B_{1}+B_{2}\right)-\left(B_{m-1}+2 B_{m}+B_{m+1}\right)$

2. $\frac{\partial^{2} \mathbf{B}}{\partial \mathbf{x}^{2}}$

In terms of difference formulae :

$B^{n+1}=B+\frac{\Delta t}{(\Delta x)^{2}}\left(B_{i+1, j}-2 B_{i, j}+B_{i-1, j}\right)$

The total axial flux is then :

$\Phi^{n+1}=\Phi+\frac{\Delta t \Delta y}{4 \Delta x}\left\{\sum_{i=1}^{m-1}\left[2 \sum_{j=2}^{n-1}+\psi_{j=1}+\psi_{j=n}\right]\right\}$

where :

$\psi=\left(B_{i+1, j}-2 B_{i, j}+B_{i-1, j}+B_{i+2, j}-2 B_{i+1, j}+B_{i, j}\right.$ 
$\left.+B_{i+1, j+1}-2 B_{i, j+1}+B_{i-1, j+1}+B_{i+2, j+1}-2 B_{i+1, j+1}+B_{i, j+1}\right)$

But :

$\sum_{i=1}^{n-1} \psi=$

$$
\begin{aligned}
& B_{0}-B_{1}-B_{2}+B_{3} \\
& +B_{1}-B_{2}-B_{3}+B_{4} \\
& +B_{2}-B_{3}-B_{4}+B_{5} \\
& +B_{3}-B_{4}-B_{5}+B_{6}
\end{aligned}
$$$$
+B_{m-5}-B_{m-4}-B_{m-3}+B_{m-2}
$$$$
+B_{m-4}-B_{m-3}-B_{m-2}+B_{m-1}
$$$$
+B_{m-3}-B_{m-2}-B_{m-1}+B_{m}
$$$$
+B_{m-2}-B_{m-1}-B_{m}+B_{m}
$$$$
=\left(B_{0}-B_{2}\right)+\left(B_{m+1}-B_{m-1}\right)
$$ 
$\Phi^{n+1}=\Phi+\frac{\Delta t \Delta y}{4 \Delta x}\left[2 \sum_{j=2}^{n-1} \Psi+\Psi_{j=1}+\Psi_{j=n}\right]$

where :

$\Psi=\left(B_{0}-B_{2}\right)+\left(B_{m+1}-B_{m-1}\right)$ 


\section{Appendix C}

\section{Boundary Conditions For Rectangular Cross-Sections}

The requirement that the tangential component of the electric field vanish at the perfectly conducting interface yields Neumann type boundary conditions. Using Ohm's Law :

$$
\begin{aligned}
E_{1} & =a F B_{1}+\frac{b}{h_{2} h_{3}}\left\{\frac{\partial}{\partial u_{3}}\left(h_{2} B_{2}\right)-\frac{\partial}{\partial u_{2}}\left(h_{3} B_{3}\right)\right\} \\
E_{2} & =a F B_{2}+\frac{b}{h_{1} h_{3}}\left\{\frac{\partial}{\partial u_{1}}\left(h_{3} B_{3}\right)-\frac{\partial}{\partial u_{3}}\left(h_{1} B_{1}\right)\right\} \\
E_{3} & =a F B_{3}+\frac{b}{h_{1} h_{2}}\left\{\frac{\partial}{\partial u_{2}}\left(h_{1} B_{1}\right)-\frac{\partial}{\partial u_{1}}\left(h_{2} B_{2}\right)\right\} \\
& \left.+\left(B_{1}^{2}+B_{2}^{2}+B_{3}^{2}\right)\right\} \\
& +\frac{B_{3}}{h_{1} h_{2}}\left\{\frac{\partial}{\partial u_{2}}\left(h_{1} B_{1}\right)-\frac{\partial}{\partial u_{1}}\left(h_{2} B_{2}\right)\right\} \\
& +\frac{B_{2}}{h_{1} h_{3}}\left\{\frac{\partial}{\partial u_{1}}\left(h_{3} B_{3}\right)-\frac{\partial}{\partial u_{3}}\left(h_{1} B_{1}\right)\right\}
\end{aligned}
$$


Consider boundaries where :

$$
\frac{\partial}{\partial u_{2}}\left(h_{2} B_{2}\right)=\frac{\partial}{\partial u_{3}}\left(h_{2} B_{2}\right)=B_{2}=E_{1}=0 \quad \text { and } \quad E_{3}=C
$$

(1) gives :

$$
\frac{\partial}{\partial u_{2}}\left(h_{1} B_{1}\right)=\frac{h_{1}\left((a-b) B_{1}^{2}-b B_{3}^{2}\right)}{a h_{3} B_{1} B_{3}} \frac{\partial}{\partial u_{2}}\left(h_{3} B_{3}\right)
$$

from (3) :

$$
\frac{\partial}{\partial u_{2}}\left(h_{1} B_{1}\right)=\frac{h_{1}\left\{a B_{1} B_{3} \frac{\partial}{\partial u_{2}}\left(h_{3} B_{3}\right)+h_{2} h_{3} C\left(B_{1}{ }^{2}+B_{3}{ }^{2}\right)\right\}}{h_{3}\left((a-b) B_{3}{ }^{2}-b B_{1}{ }^{2}\right)}
$$

(4) and (5) then give :

$$
\begin{aligned}
& \frac{\partial}{\partial u_{2}}\left(h_{3} B_{3}\right)=\frac{-h_{2} h_{3} C a B_{1} B_{3}}{b(a-b)\left(B_{1}^{2}+B_{3}^{2}\right)} \\
& \frac{\partial}{\partial u_{2}}\left(h_{1} B_{1}\right)=\frac{-h_{1} h_{2} C\left((a-b) B_{1}^{2}-b B_{3}^{2}\right)}{b(a-b)\left(B_{1}^{2}+B_{3}^{2}\right)}
\end{aligned}
$$

Substituting for $\mathrm{a}, \mathrm{b}$ and redefining $\mathrm{C}$ yields :

$$
\begin{aligned}
& \frac{\partial}{\partial u_{2}}\left(h_{3} B_{3}\right)=\frac{h_{2} h_{3} \alpha(1-\varepsilon) B_{1} B_{3}}{B_{1}{ }^{2}+B_{3}{ }^{2}} \\
& \frac{\partial}{\partial u_{2}}\left(h_{1} B_{1}\right)=\frac{-h_{1} h_{2} \alpha\left(\varepsilon B_{1}^{2}+B_{3}^{2}\right)}{B_{1}^{2}+B_{3}{ }^{2}}
\end{aligned}
$$


Now consider boundaries where :

$$
\frac{\partial}{\partial u_{2}}\left(h_{1} B_{2}\right)=\frac{\partial}{\partial u_{3}}\left(h_{1} B_{2}\right)=B_{1}=E_{2}=0 \quad \text { and } \quad E_{3}=C
$$

(2) gives :

$$
\frac{\partial}{\partial u_{1}}\left(h_{2} B_{2}\right)=\frac{h_{1}\left((a-b) B_{1}^{2}-b B_{3}^{2}\right)}{a h_{3} B_{1} B_{3}} \frac{\partial}{\partial u_{2}}\left(h_{3} B_{3}\right)
$$

from (3) :

$$
\frac{\partial}{\partial u_{1}}\left(h_{2} B_{2}\right)=\frac{h_{2}\left\{a B_{2} B_{3} \frac{\partial}{\partial u_{1}}\left(h_{3} B_{3}\right)-h_{1} h_{3} C\left(B_{2}{ }^{2}-B_{3}{ }^{2}\right)\right\}}{h_{3}\left((a-b) B_{3}^{2}-b B_{2}{ }^{2}\right)}
$$

(6) and (7) then yields :

$$
\begin{aligned}
& \frac{\partial}{\partial u_{1}}\left(h_{3} B_{3}\right)=\frac{h_{1} h_{3} C a B_{2} B_{3}}{b(a-b)\left(B_{2}{ }^{2}+B_{3}{ }^{2}\right)} \\
& \frac{\partial}{\partial u_{1}}\left(h_{2} B_{2}\right)=\frac{h_{1} h_{2} C\left((a-b) B_{2}^{2}-b B_{3}^{2}\right)}{b(a-b)\left(B_{2}{ }^{2}+B_{3}^{2}\right)}
\end{aligned}
$$

substituting for $\mathrm{a}$ and $\mathrm{b}$ :

$$
\begin{aligned}
& \frac{\partial}{\partial u_{1}}\left(h_{3} B_{3}\right)=\frac{-h_{1} h_{3} \alpha(1-\varepsilon) B_{2} B_{3}}{B_{2}{ }^{2}+B_{3}{ }^{2}} \\
& \frac{\partial}{\partial u_{1}}\left(h_{2} B_{2}\right)=\frac{h_{1} h_{2} \alpha\left(\varepsilon B_{2}^{2}+B_{3}^{2}\right)}{B_{2}{ }^{2}+B_{3}{ }^{2}}
\end{aligned}
$$




\section{Cartesians}

Set :

$$
h_{1}=h_{2}=h_{3}=1 \quad \text { and } \quad u 1=x, u 2=y \text { and } u 3=z
$$

then on boundaries where :

$$
\frac{\partial B_{y}}{\partial x}=\frac{\partial B_{y}}{\partial z}=B_{y}=E_{x}=0 \text { and } E_{z}=C
$$

the following must be satisfied :

$$
\begin{aligned}
& \frac{\partial B_{z}}{\partial y}=\frac{\alpha(1-\varepsilon) B_{x} B_{y}}{B_{x}^{2}+B_{z}^{2}} \\
& \frac{\partial B_{x}}{\partial y}=\frac{-\alpha\left(\varepsilon B_{x}^{2}+B_{z}^{2}\right)}{B_{x}^{2}+B_{z}^{2}}
\end{aligned}
$$

on boundaries where :

$$
\frac{\partial B_{x}}{\partial y}=\frac{\partial B_{x}}{\partial z}=B_{x}=E_{y}=0 \quad \text { and } \quad E_{z}=C
$$

the following must be satisfied :

$$
\begin{aligned}
& \frac{\partial B_{z}}{\partial x}=\frac{-\alpha(1-\varepsilon) B_{y} B_{z}}{b_{y}{ }^{2}+B_{z}^{2}} \\
& \frac{\partial B_{y}}{\partial x}=\frac{\alpha\left(\varepsilon B_{y}^{2}+B_{z}^{2}\right)}{b_{y}^{2}+B_{z}^{2}}
\end{aligned}
$$




\section{Toroidal co-ordinates}

Set :

$$
h_{1}=h_{2}=1, h_{3}=R=R_{o}+x \quad \text { and } \quad u 1=x, u 2=y \quad \text { and } u 3=\phi
$$

then on boundaries where :

$$
\frac{\partial B_{y}}{\partial x}=\frac{\partial B_{y}}{\partial \phi}=B_{y}=E_{x}=0 \text { and } E_{\phi}=C
$$

the following must be satisfied :

$$
\begin{aligned}
& \frac{\partial}{\partial y}\left(R B_{\phi}\right)=\frac{\alpha R(1-\varepsilon) B_{x} B_{y}}{B_{x}^{2}+B_{\phi}^{2}} \\
& \frac{\partial B_{x}}{\partial y}=\frac{-\alpha\left(\varepsilon B_{x}^{2}+B_{\phi}^{2}\right)}{B_{x}^{2}+B_{\phi}^{2}}
\end{aligned}
$$

on boundaries where :

$$
\frac{\partial B_{x}}{\partial y}=\frac{\partial B_{x}}{\partial \phi}=B_{x}=E_{y}=0 \text { and } E_{\circ}=C
$$

the following must be satisfied :

$$
\begin{aligned}
& \frac{\partial}{\partial x}\left(R B_{\phi}\right) \frac{-\alpha R(1-\varepsilon) B_{y} B_{\phi}}{b_{y}^{2}+B_{\phi}^{2}} \\
& \frac{\partial B_{y}}{\partial x}=\frac{\alpha\left(\varepsilon B_{y}^{2}+B_{\phi}^{2}\right)}{b_{y}^{2}+B_{\phi}^{2}}
\end{aligned}
$$




\section{Boundary Conditions for Arbitrary Cross-sections}

Consider a boundary which does not necessarily conform to any particular coordinate curve. In such cases the boundary conditions must be obtained in a particular co-ordinate system as the scale factors are in general incalculable. Cartesians co-ordinates will be used throughout.

The requirement that the normal component of the magnetic field and the tangential component of the electric field vanish at the boundary can be written as :

$$
\begin{aligned}
& B_{x} \cos \beta+B_{y} \sin \beta=0 \\
& E_{x} \sin \beta-E_{y} \cos \beta=0
\end{aligned}
$$

Substituting from O'hms Law and assuming a constant driving field leads to:

$$
\begin{aligned}
& B_{x}+B_{y} \tan \beta=0 \\
& \left\{a F B_{y}-b \frac{\partial B_{z}}{\partial x}\right\}=\left\{a F B_{x}+b \frac{\partial B_{z}}{\partial y}\right\} \tan \beta \\
& a F B_{z}+b\left\{\frac{\partial B_{y}}{\partial x}-\frac{\partial B_{x}}{\partial y}\right\}=C
\end{aligned}
$$

from (2)

$$
\frac{\partial B_{z}}{\partial x}+T \frac{\partial B_{z}}{\partial y}=\frac{a B_{y} B_{z}\left(1+T^{2}\right)}{B_{y}^{2}\left(1+T^{2}\right)(a-b)-b B_{z}^{2}}\left\{\frac{\partial B_{y}}{\partial x}-\frac{\partial B_{x}}{\partial y}\right\}
$$

from (3)

$$
a B_{y} B_{z}\left\{\frac{\partial B_{z}}{\partial x}+T \frac{\partial B_{z}}{\partial y}\right\}=\left(b B_{y}^{2}\left(1+T^{2}\right)-B_{z}^{2}(a-b)\right)\left\{\frac{\partial B_{x}}{\partial y}-\frac{\partial B_{y}}{\partial x}\right\}
$$




$$
+C\left(B_{y}{ }^{2}\left(1+T^{2}\right)+B_{z}{ }^{2}\right)
$$

from which follow :

$$
\begin{aligned}
& \frac{\partial B_{y}}{\partial x}=\frac{\partial B_{x}}{\partial y}+\frac{C\left(B_{y}{ }^{2}\left(1+T^{2}\right)(a-b)-b B_{z}{ }^{2}\right)}{b(a-b)\left(B_{y}{ }^{2}\left(1+T^{2}\right)+B_{z}{ }^{2}\right)} \\
& \frac{\partial B_{z}}{\partial x}+T \frac{\partial B_{z}}{\partial y}=\frac{a C B_{y} B_{z}\left(1+T^{2}\right)}{b(a-b)\left(B_{y}{ }^{2}\left(1+T^{2}\right)+B_{z}{ }^{2}\right)}
\end{aligned}
$$

after substituting for $\mathrm{a}$ and $\mathrm{b}$ :

$$
\begin{aligned}
& \frac{\partial B_{y}}{\partial x}=\frac{\partial B_{x}}{\partial y}+\alpha \frac{\left(\varepsilon B_{y}{ }^{2}\left(1+T^{2}\right)+B_{z}{ }^{2}\right)}{\left(B_{y}{ }^{2}\left(1+T^{2}\right)+B_{z}{ }^{2}\right)} \\
& \frac{\partial B_{z}}{\partial x}+T \frac{\partial B_{z}}{\partial y}=\alpha \frac{B_{y} B_{z}(\varepsilon-1)\left(1+T^{2}\right)}{\left(B_{y}{ }^{2}\left(1+T^{2}\right)+B_{z}{ }^{2}\right)}
\end{aligned}
$$




\section{Appendix D}

Numerical value of $\nabla \cdot \mathbf{B}$ in curvilinear co-ordinates

Consider the evolution equations in the following form :

$$
\begin{aligned}
& \frac{1}{a 1} \frac{\partial B_{1}}{\partial t}=\left[\frac{\partial}{\partial u_{2}}\left[a \frac{\partial}{\partial u_{2}}\left(B_{1}\right)\right]-\frac{\partial}{\partial u_{2}}\left(a \frac{\partial}{\partial u_{1}}\left(B_{2}\right)\right]\right. \\
& +\frac{\partial}{\partial u_{3}}\left(c \frac{\partial}{\partial u_{3}}\left(B_{1}\right)\right]-\frac{\partial}{\partial u_{3}}\left(c \frac{\partial}{\partial u_{1}}\left(B_{3}\right)\right) \\
& \left.-\beta\left\{\frac{\partial}{\partial u_{2}}(C 3)-\frac{\partial}{\partial u_{3}}(C 2)\right\}\right] \\
& \frac{1}{a 2} \frac{\partial B_{1}}{\partial t}=\left[\frac{\partial}{\partial u_{3}}\left[b \frac{\partial}{\partial u_{3}}\left(B_{2}\right)\right]-\frac{\partial}{\partial u_{3}}\left[b \frac{\partial}{\partial u_{2}}\left(B_{3}\right)\right]\right. \\
& +\frac{\partial}{\partial u_{1}}\left[a \frac{\partial}{\partial u_{1}}\left(B_{2}\right)\right]-\frac{\partial}{\partial u_{1}}\left[a \frac{\partial}{\partial u_{1}}\left(B_{1}\right)\right] \\
& \left.-\beta\left\{\frac{\partial}{\partial u_{3}}(C 1)-\frac{\partial}{\partial u_{1}}(C 3)\right\}\right] \\
& \frac{1}{a 3} \frac{\partial B_{1}}{\partial t}=\left[\frac{\partial}{\partial u_{1}}\left(c \frac{\partial}{\partial u_{1}}\left(B_{3}\right)\right]-\frac{\partial}{\partial u_{1}}\left(c \frac{\partial}{\partial u_{3}}\left(B_{1}\right)\right]\right. \\
& +\frac{\partial}{\partial u_{2}}\left[b \frac{\partial}{\partial u_{2}}\left(B_{3}\right)\right]-\frac{\partial}{\partial u_{2}}\left[b \frac{\partial}{\partial u_{3}}\left(B_{2}\right)\right]
\end{aligned}
$$




$$
\left.-\beta\left\{\frac{\partial}{\partial u_{1}}(C 2)-\frac{\partial}{\partial u_{2}}(C 1)\right\}\right]
$$

Where :

$$
\begin{aligned}
& a 1=\frac{1}{h_{2} h_{3}} ; a 2=\frac{1}{h_{1} h_{3}} ; a 3=\frac{1}{h_{1} h_{2}} ; \\
& a=a 3 h_{3 ;} \quad b=a 1 h_{1} ; \text { and } c=a 2 h_{2}
\end{aligned}
$$

Then in terms of curvilinear co-ordinates the divergence-free constraint can be written as :

$$
\begin{aligned}
\nabla \cdot \mathbf{B} & =\frac{1}{h_{1} h_{2} h_{3}}\left[\frac{\partial}{\partial u_{1}}\left(\frac{B_{1}}{a 1}\right)+\frac{\partial}{\partial u_{2}}\left(\frac{B_{2}}{a 2}\right)+\frac{\partial}{\partial u_{3}}\left(\frac{B_{3}}{a 3}\right)\right]=0 \\
& =\frac{1}{h_{1} h_{2} h_{3}}\left(D_{1}+D_{2}+D_{3}\right)
\end{aligned}
$$

Now use the following difference representations :

$$
\begin{aligned}
& \frac{\partial U}{\partial x}=\frac{U_{i+1}-U_{i-1}}{2 \Delta x} \\
& \frac{\partial}{\partial x}\left[p \frac{\partial U}{\partial x}\right]=\frac{p_{i+1}\left(U_{i+2}-U_{i}\right)-p_{i-1}\left(U_{i-2}-U_{i}\right)}{4 \Delta x^{2}} \\
& \frac{\partial}{\partial x}\left[p \frac{\partial U}{\partial y}\right]=\frac{p_{i+1}\left(U_{i+1, j+1}-U_{i+1, j-1}\right)-p_{i-1}\left(U_{i-1, j+1}-U_{i-1, j-1}\right)}{4 \Delta x \Delta y}
\end{aligned}
$$


The evolution equations can then be written as :

$$
\begin{aligned}
& \frac{B_{1}^{n+1}-B_{1}}{a 1 \Delta t}= \\
& {\left[a_{j+1}\left(\left(B_{1}\right)_{j+2}-\left(B_{1}\right)_{j}\right)-a_{j-1}\left(\left(B_{1}\right)_{j}-\left(B_{1}\right)_{j-2}\right)\right] \div 4 \Delta_{2}^{2}} \\
& -\left[a_{j+1}\left(\left(B_{2}\right)_{i+1, j+1}-\left(B_{2}\right)_{i-1, j+1}\right)-a_{j-1}\left(\left(B_{2}\right)_{i+1, j-1}-\left(B_{2}\right)_{i-1, j-1}\right)\right] \div 4 \Delta_{1} \Delta_{2} \\
& +\left[c_{k+1}\left(\left(B_{1}\right)_{k+2-}\left(B_{1}\right)_{k}\right)-c_{k-1}\left(\left(B_{1}\right)_{k}-\left(B_{1}\right)_{k-2}\right)\right] \div 4 \Delta_{3}^{2} \\
& -\left[c_{k+1}\left(\left(B_{2}\right)_{i+1, k+1}-\left(B_{2}\right)_{i-1, k+1}\right)-c_{k-1}\left(\left(B_{2}\right)_{i+1, k-1}-\left(B_{2}\right)_{i-1, k-1}\right)\right] \div 4 \Delta_{1} \Delta_{3} \\
& -\beta\left(C 3_{j+1}-C 3_{j-1}\right) \div 2 \Delta_{2} \\
& +\beta\left(C 2_{k+1}-C 2_{k-1}\right) \div 2 \Delta_{3} \\
& \frac{B_{2}^{n+1}-B_{2}}{a 2 \Delta t}= \\
& {\left[b_{k+1}\left(\left(B_{2}\right)_{k+2}-\left(B_{2}\right)_{k}\right)-b_{k-1}\left(\left(B_{2}\right)_{k}-\left(B_{2}\right)_{k-2}\right)\right] \div 4 \Delta_{3}^{2}} \\
& -\left[b_{k+1}\left(\left(B_{2}\right)_{j+1, k+1}-\left(B_{2}\right)_{j-1, k+1}\right)-b_{k-1}\left(\left(B_{2}\right)_{j+1, k-1}-\left(B_{2}\right)_{j-1, k-1}\right)\right] \div 4 \Delta_{2} \Delta_{3} \\
& +\left[a_{i+1}\left(\left(B_{2}\right)_{i+2-}\left(B_{2}\right)_{i}\right)-a_{i-1}\left(\left(B_{2}\right)_{i}-\left(B_{2}\right)_{i-2}\right)\right] \div 4 \Delta_{1}^{2} \\
& -\left[a_{i+1}\left(\left(B_{2}\right)_{i+1, j+1}-\left(B_{2}\right)_{i+1, j-1}\right)-a_{i-1}\left(\left(B_{2}\right)_{i-1, j+1}-\left(B_{2}\right)_{i-1, j-1}\right)\right] \div 4 \Delta_{1} \Delta_{2}
\end{aligned}
$$


$-\beta\left(C 1_{k+1}-C 1_{k-1}\right) \div 2 \Delta_{3}$

$+\beta\left(C 3_{i+1}-C 3_{i-1}\right) \div 2 \Delta_{x}$

$\frac{B_{3}^{n+1}-B_{3}}{a 3 \Delta t}=$

$\left[c_{i+1}\left(\left(B_{3}\right)_{i+2}-\left(B_{3}\right)_{i}\right)-c_{i-1}\left(\left(B_{3}\right)_{i}-\left(B_{3}\right)_{i-2}\right)\right] \div 4 \Delta_{2}^{2}$

$-\left[c_{i+1}\left(\left(B_{1}\right)_{i+1, k+1}-\left(B_{1}\right)_{i+1, k-1}\right)-c_{i-1}\left(\left(B_{1}\right)_{i-1, k+1}-\left(B_{1}\right)_{i-1, k-1}\right)\right] \div 4 \Delta_{1} \Delta_{2}$

$+\left[b_{j+1}\left(\left(B_{3}\right)_{j+2-}\left(B_{3}\right)_{j}\right)-b_{j-1}\left(\left(B_{3}\right)_{j}-\left(B_{3}\right)_{j-2}\right)\right]+4 \Delta_{3}^{2}$

$-\left[b_{j+1}\left(\left(B_{2}\right)_{j+1, k+1}-\left(B_{2}\right)_{j+1, k-1}\right)-b_{j-1}\left(\left(B_{2}\right)_{j-1, k+1}-\left(B_{2}\right)_{j-1, k-1}\right)\right] \div 4 \Delta_{1} \Delta_{3}$

$-\beta\left(C 2_{i+1}-C 2_{i-1}\right) \div 2 \Delta_{1}$

$+\beta\left(C 1_{j+1}-C 1_{j-1}\right) \div 2 \Delta_{2}$

The $\nabla \cdot \mathbf{B}$ terms then become :

$D_{1}^{n+1}=D_{1}+$

$\left[\left(a_{i+1, j+1}\left(\left(B_{1}\right)_{i+1, j+2}-\left(B_{1}\right)_{i+1, j}\right)-\left(a_{i+1, j-1}\left(\left(B_{1}\right)_{i+1, j}-\left(B_{1}\right)_{i+1, j-2}\right)\right.\right.\right.$

$-\left(a_{i-1, j+1}\left(\left(B_{1}\right)_{i-1, j+2}-\left(B_{1}\right)_{i-1, j}\right)+\left(a_{i-1, j-1}\left(\left(B_{1}\right)_{i-1, j}-\left(B_{1}\right)_{i-1, j-2}\right)\right] \div 8 \Delta_{2}^{2} \Delta_{1}\right.$ 


$$
\begin{aligned}
& {\left[\left(a_{i+1, j+1}\left(\left(B_{2}\right)_{i+2, j+1}-\left(B_{2}\right)_{i, j+1}\right)-\left(a_{i-1, j+1}\left(\left(B_{2}\right)_{i+2, j-1}-\left(B_{2}\right)_{i, j-1}\right)\right.\right.\right.} \\
& -\left(a_{i-1, j+1}\left(\left(B_{2}\right)_{i, j+1}-\left(B_{2}\right)_{i-2, j+1}\right)-\left(a_{i-1, j-1}\left(\left(B_{2}\right)_{i, j-1}-\left(B_{2}\right)_{i-2, j-1}\right)\right] \div 8 \Delta_{1}^{2} \Delta_{2}\right. \\
& +\left[\left(a_{i+1, k+1}\left(\left(B_{1}\right)_{i+1, k+2}-\left(B_{1}\right)_{i+1, k}\right)-\left(a_{i+1, k-1}\left(\left(B_{1}\right)_{i+1, k}-\left(B_{1}\right)_{i+1, k-2}\right)\right.\right.\right. \\
& -\left(a_{i-1, k+1}\left(\left(B_{1}\right)_{i-1, k+2}-\left(B_{1}\right)_{i-1, k}\right)-\left(a_{i-1, k-1}\left(\left(B_{1}\right)_{i-1, k}-\left(B_{1}\right)_{i-1, k-2}\right)\right] \div 8 \Delta_{3}^{2} \Delta_{1}\right.
\end{aligned}
$$$$
\left[\left(a_{i+1, k+1}\left(\left(B_{3}\right)_{i+2, k+1}-\left(B_{3}\right)_{i, k+1}\right)-\left(a_{i-1, k+1}\left(\left(B_{3}\right)_{i+2, k-1}-\left(B_{3}\right)_{i, k-1}\right)\right.\right.\right.
$$$$
-\left(a_{i-1, k+1}\left(\left(B_{3}\right)_{i, k+1}-\left(B_{3}\right)_{i-2, k+1}\right)-\left(a_{i-1, k-1}\left(\left(B_{3}\right)_{i, k-1}-\left(B_{3}\right)_{i-2, k-1}\right)\right] \div 8 \Delta_{1}^{2} \Delta_{3}\right.
$$$$
-\beta\left[C 3_{i+1, j+1}-C 3_{i+1, j-1}-C 3_{i-1, j+1}+C 3_{i-1, j-1}\right] \div 4 \Delta_{1} \Delta_{2}
$$$$
+\beta\left[C 2_{i+1, k+1}-C 2_{i+1, k-1}-C 2_{i-1, k+1}+C 2_{i-1, k-1}\right] \div 4 \Delta_{1} \Delta_{3}
$$$$
D_{2}^{n+1}=D_{2}+
$$$$
\left[\left(b_{i+1, k+1}\left(\left(B_{1}\right)_{i+1, k+2}-\left(B_{1}\right)_{i+1, k}\right)-\left(b_{i+1, k-1}\left(\left(B_{1}\right)_{i+1, k}-\left(B_{1}\right)_{i+1, k-2}\right)\right.\right.\right.
$$$$
-\left(b_{i-1, k+1}\left(\left(B_{1}\right)_{i-1, k+2}-\left(B_{1}\right)_{i-1, k}\right)+\left(b_{i-1, k-1}\left(\left(B_{1}\right)_{i-1, k}-\left(B_{1}\right)_{i-1, k-2}\right)\right] \div 8 \Delta_{3}^{2} \Delta_{2}\right.
$$$$
\left[\left(b_{j+1, k+1}\left(\left(B_{2}\right)_{j+2, k+1}-\left(B_{2}\right)_{j, k+1}\right)-\left(b_{j-1, k+1}\left(\left(B_{2}\right)_{j+2, k-1}-\left(B_{2}\right)_{j, k-1}\right)\right.\right.\right.
$$$$
-\left(b_{j-1, k+1}\left(\left(B_{2}\right)_{j, k+1}-\left(B_{2}\right)_{j-2, k+1}\right)-\left(b_{j-1, k-1}\left(\left(B_{2}\right)_{j, k-1}-\left(B_{2}\right)_{j-2, k-1}\right)\right] \div 8 \Delta_{2}^{2} \Delta_{3}\right.
$$ 


$$
\begin{aligned}
& +\left[\left(a_{i+1, j+1}\left(\left(B_{1}\right)_{i+2, j+1}-\left(B_{1}\right)_{i, j+1}\right)-\left(a_{i-1, j+1}\left(\left(B_{1}\right)_{i, j+1}-\left(B_{1}\right)_{i-2, j+1}\right)\right.\right.\right. \\
& -\left(a_{i+1, j-1}\left(\left(B_{1}\right)_{i+2, j-1}-\left(B_{1}\right)_{i, j-1}\right)-\left(a_{i-1, j-1}\left(\left(B_{1}\right)_{i, j-1}-\left(B_{1}\right)_{i-2, j-1}\right)\right]+8 \Delta_{1}^{2} \Delta_{2}\right. \\
& {\left[\left(a_{i+1, j+1}\left(\left(B_{3}\right)_{i+1, j+2}-\left(B_{3}\right)_{i+1, j}\right)-\left(a_{i-1, j+1}\left(\left(B_{3}\right)_{i-1, j+2}-\left(B_{3}\right)_{i-1, j}\right)\right.\right.\right.} \\
& -(i+1, j-1 \\
& \left(\left(B_{3}\right)_{i+1, j}-\left(B_{3}\right)_{i+1, j-2}\right)-\left(a_{i-1, j-1}\left(\left(B_{3}\right)_{i-1, j}-\left(B_{3}\right)_{i-1, j-2}\right)\right] \div 8 \Delta_{1}^{2} \Delta_{1}
\end{aligned}
$$$$
-\beta\left[C 1_{j+1, k+1}-C 1_{j+1, k-1}-C 1_{j-1, k+1}+C 1_{j-1, k-1}\right] \div 4 \Delta_{2} \Delta_{3}
$$$$
+\beta\left[C 3_{i+1, j+1}-C 3_{i+1, j-1}-C 3_{i-1, j+1}+C 3_{i-1, j-1}\right] \div 4 \Delta_{1} \Delta_{2}
$$$$
D_{3}^{n+1}=D_{3}+
$$$$
\left[\left(c_{i+1, k+1}\left(\left(B_{3}\right)_{i+2, k+2}-\left(B_{3}\right)_{i, k+1}\right)-\left(c_{i-1, k+1}\left(\left(B_{3}\right)_{i, k+1}-\left(B_{3}\right)_{i-2, k+1}\right)\right.\right.\right.
$$$$
-\left(c_{i+1, k-1}\left(\left(B_{3}\right)_{i+2, k-1}-\left(B_{3}\right)_{i, k-1}\right)+\left(c_{i+1, k-1}\left(\left(B_{3}\right)_{i, k-1}-\left(B_{3}\right)_{i-2, k-1}\right)\right] \div 8 \Delta_{1}^{2} \Delta_{3}\right.
$$$$
\left[\left(c_{i+1, k+1}\left(\left(B_{1}\right)_{i+1, k+2}-\left(B_{1}\right)_{i+1, k}\right)-\left(c_{i-1, k+1}\left(\left(B_{1}\right)_{i-1, k+2}-\left(B_{1}\right)_{i-1, k}\right)\right.\right.\right.
$$$$
-\left(c_{i+1, k-1}\left(\left(B_{1}\right)_{i+1, k}-\left(B_{1}\right)_{i+1, k-2}\right)-\left(c_{i-1, k-1}\left(\left(B_{1}\right)_{i-1, k}-\left(B_{1}\right)_{i-1, k-2}\right)\right] \div 8 \Delta_{3}^{2} \Delta_{1}\right.
$$$$
+\left[\left(b_{j+1, k+1}\left(\left(B_{3}\right)_{j+2, k+1}-\left(B_{3}\right)_{j, k+1}\right)-\left(b_{j-1, k+1}\left(\left(B_{3}\right)_{j, k+1}-\left(B_{3}\right)_{j-2, k+1}\right)\right.\right.\right.
$$$$
-\left(b_{j+1, k-1}\left(\left(B_{3}\right)_{j+2, k-1}-\left(B_{3}\right)_{j, k-1}\right)-\left(b_{j-1, k-1}\left(\left(B_{3}\right)_{j, k-1}-\left(B_{3}\right)_{j-2, k-1}\right)\right] \div 8 \Delta_{2}^{2} \Delta_{3}\right.
$$ 


$$
\begin{aligned}
& {\left[\left(b_{j+1, k+1}\left(\left(B_{2}\right)_{j+1, k+2}-\left(B_{2}\right)_{j+1, k}\right)-\left(b_{j-1, k+1}\left(\left(B_{2}\right)_{j-1, k+2}-\left(B_{2}\right)_{j-1, k}\right)\right.\right.\right.} \\
& -\left(b_{j+1, k-1}\left(\left(B_{2}\right)_{j+1, k}-\left(B_{2}\right)_{j+1, k-2}\right)-\left(b_{j-1, k-1}\left(\left(B_{2}\right)_{j-1, k}-\left(B_{2}\right)_{j-1, k-2}\right)\right] \div 8 \Delta_{3}^{2} \Delta_{2}\right. \\
& -\beta\left[C 2_{i+1, k+1}-C 2_{i-1, k+1}-C 2_{i+1, k-1}+C 2_{i-1, k-1}\right] \div 4 \Delta_{1} \Delta_{3} \\
& +\beta\left[C 1_{j+1, k+1}-C 1_{j-1, k+1}-C 1_{j+1, k-1}+C 1_{j-1, k-1}\right] \div 4 \Delta_{2} \Delta_{3}
\end{aligned}
$$

Adding these shows that $\nabla \cdot \mathbf{B}$ at the advanced time-step is the the same as that at the preceding time-step. If $\nabla \cdot \mathbf{B}$ is zero initially it will then remain so for subsequent times. This is only true provided the adjacent points to that being considered are updated using the evolution equations. This will obviously cause problems at points which are interior boundary neighbours as the requirement that the normal component of the magnetic field vanish at the interface are used to assign values at the boundary.

\section{The divergence of $B$ at interior boundary neighbours}

Consider the interior boundary neighbours to the top-most boundary. The value of $B_{y}$ is set to zero at the boundary and so the non-vanishing term is :

$$
\begin{aligned}
& {\left[b \frac{\partial B_{3}}{\partial u_{2}}+(1-\varepsilon) C 1\right]_{k-1}^{k+1} \div 4 \Delta_{2} \Delta_{3}} \\
& +\left[a \frac{\partial B_{1}}{\partial u_{2}}-(1-\varepsilon) C 3\right]_{i-1}^{i+1} \div 4 \Delta_{1} \Delta_{2}
\end{aligned}
$$

On the boundary :

$$
\frac{\partial B_{1}}{\partial u_{2}}=-h_{1} h_{2} \alpha \frac{\left(\varepsilon B_{1}^{2}+B_{3}^{2}\right)}{\left(B_{1}^{2}+B_{3}^{2}\right)}
$$




$$
\begin{aligned}
& \frac{\partial B_{3}}{\partial u_{2}}=h_{2} h_{3} \alpha \frac{(1-\varepsilon) B_{1} B_{3}}{\left(B_{1}{ }^{2}+B_{3}{ }^{2}\right)} \\
& C 1=h_{1} F B_{1}=-\frac{h_{1} \alpha B_{1} B_{3}}{\left(B_{1}{ }^{2}+B_{3}{ }^{2}\right)} \\
& C 3=h_{3} F B_{3}=-\frac{h_{3} \alpha B_{3}{ }^{2}}{\left(B_{1}{ }^{2}+B_{3}{ }^{2}\right)}
\end{aligned}
$$

Substituting into the above term yields only :

$$
\frac{-\alpha \varepsilon}{4 \Delta_{1} \Delta_{2}}\left[h_{3}\right]_{i-1}^{i+1}=-\frac{\alpha \varepsilon}{2 \Delta_{2}}
$$

Now consider interior boundary neighbours to the LHS boundary. The value of $B_{x}$ is set to zero at the boundary and so the non-vanishing term is :

$$
\begin{array}{r}
{\left[a \frac{\partial B_{2}}{\partial u_{1}}+(1-\varepsilon) C 3\right]_{j-1}^{j+1} \div 4 \Delta_{1} \Delta_{2}} \\
+\left[c \frac{\partial B_{3}}{\partial u_{1}}-(1-\varepsilon) C 2\right]_{k-1}^{k+1} \div 4 \Delta_{1} \Delta_{3}
\end{array}
$$

On the boundary :

$$
\begin{aligned}
& \frac{\partial B_{2}}{\partial u_{1}}=h_{1} h_{2} \alpha \frac{\left(\varepsilon B_{2}^{2}+B_{3}^{2}\right)}{\left(B_{2}{ }^{2}+B_{3}{ }^{2}\right)} \\
& \frac{\partial B_{3}}{\partial u_{2}}=h_{1} h_{3} \alpha \frac{(\varepsilon-1) B_{2} B_{3}}{\left(B_{2}^{2}+B_{3}{ }^{2}\right)}
\end{aligned}
$$




$$
\begin{aligned}
& C 2=h_{2} F B_{2}=-\frac{h_{2} \alpha B_{2} B_{3}}{\left(B_{2}{ }^{2}+B_{3}{ }^{2}\right)} \\
& C 3=h_{3} F B_{3}=-\frac{h_{3} \alpha B_{3}^{2}}{\left(B_{2}^{2}+B_{3}^{2}\right)}
\end{aligned}
$$

Substituting into the above term yields only :

$$
\frac{\alpha \varepsilon}{4 \Delta_{1} \Delta_{2}}\left[h_{3}\right]_{i-1}^{i+1}
$$

In Cartesian co-ordinates these are zero and so $\nabla \cdot \mathbf{B}=0$ at the advanced time is true everywhere. In Toroidal co-ordinates $h_{3}=R=R_{0}+x$ and so the non vanishing term is zero for the top-most interior boundary neighbours but is finite for the LHS ones. 


\section{References}

[1.1] M. O. Hagler and M. Kristiansen, An Introduction to Controlled Thermonuclear Fusion, Lexington Books (1977).

[1.2] D. C. Robinson et al, Plasma Physics and Nuclear Fusion Research, Academic Press LTD (1981).

[1.3] J. D. Lawson, Proc. Phys. Soc. B, 70, 6 (1957).

[1.4] J. A. Bittencourt, Fundamentals of Plasma Physics, Pergamon Press (1986).

[1.5] T. J. M. Boyd and J. J. Sanderson, Plasma Dynamics, Thomas Nelson and Sons LTD (1969).

[1.6] G. Bateman, MHD Instabilities, The MIT Press, (1980).

[1.7] Private Communications.

[1.8] G. D. Hobbs, Culham Lab. Report, CLM-P19 (1963).

[1.9] L. Woltjer, Proc. Nat. Acad. Sci., 44, 489 (1958).

[1.10] J. B. Taylor, Phys. Rev. Lett., 33, 1139 (1974).

[1.11] H. A. B. Bodin and A. A. Newton, Nuc. Fus., 20, 1255 (1980).

[1.12] H. A. B. Bodin, Proc. of the Int. Conf. on Plasma Phys., Vol. 1, 417 (1984).

[1.13] A. R. Mitchell and D. F. Griffiths, The Finite Difference Method in Partial Differential Equations, John Wiley and sons (1980). 
[1.14] G. D. Smith, Numerical Solution of Partial Differential Equations, Oxford University Press (1978).

[1.15] D. Potter, Computational Physics, John Wiley and sons (1973).

[1.16] K. V. Roberts and D. E. Potter, Methods in Computational Physics, Vol. 9, Academic Press, (1970).

[2.1] A. Sykes and J. A. Wesson, Phys. Rev. Letters, 37, 140 (1976).

[2.2] S. H. Douglas and D. D. Schnack, Phys. Fluids, 31, 1979 (1988).

[5.1] C. G. Gimblett et al, Phys. Fluids, 30, 3186 (1987).

[5.2] P. Kirby, Phys. Fluids, 31, 625 (1988).

[5.3] I. H. Hutchison, Phys. Rev. Letters, 37, 338, (1976).

[5.4] J. B. Taylor, Rev. Mod. Phys., 58, 741 (1986).

[5.5] K. J. Whiteman, Plasma Phys., 7, 293 (1965).

[5.6] V. D. Shafranov, Reviews of Plasma Physics, Vol. 2, Pg 103, Consultants Bureau (1970).

[5.7] J. B. Taylor, Pulsed High Beta Plasmas, Pergamon Press (1976).

[5.8] E. Kreyszig, Advanced Engineering Mathematics, John Wiley and sons (1988).

[5.9] S. Chandrasekhar and P. C. Kendall, Astrophysics J., 126, 457 (1957). 